\title{
Diferenciação de medidas em Espaços Métricos
}

\author{
Juan David Cabrera Cuellar \\ DISSERTAÇÃO APRESENTADA \\ $\mathrm{AO}$ \\ Instituto DE MATEMÁticA E EstatísticA \\ DA \\ UNIVERSIDADE DE SÃo PAUlO \\ PARA \\ OBTENÇÃO DO TÍTULO \\ DE \\ Mestre em CiÊnCIAS \\ Programa: Matemática \\ Orientador: Prof. Dr. Gláucio Terra
}

Durante o desenvolvimento deste trabalho, o autor recebeu auxílio financeiro da $\mathrm{CNPq}$

São Paulo, maio de 2021 


\section{Diferenciação de medidas em Espaços Métricos}

Esta versão da dissertação contém as correções e alterações sugeridas pela Comissão Julgadora durante a defesa da versão original do trabalho, realizada em 10/05/2021. Uma cópia da versão original está disponível no

Instituto de Matemática e Estatística da Universidade de São Paulo.

Comissão Julgadora:

- Prof. Dr. Gláucio Terra (orientador) - IME-USP

- Prof. Dr. Márcio Fabiano da Silva - UFABC

- Prof. Dr. Marcus Marrocos - UFABC 


\section{Agradecimentos}

Gostaria de agradecer a Glaucio Terra, meu orientador, pela dedicação, disposição e paciência durante o mestrado. A meus amigos e colegas, aprecio muito sua amizade e convivência durante todo esse tempo. Por ultimo, agradeço a minha família pelo apoio e compreensão em todo momento. Gracias totales. 


\section{Resumo}

CABRERA, J. D. C. Diferenciação de medidas em espaços métricos. 2020. 63 p. Dissertação (Mestrado) - Instituto de Matemática e Estatística, Universidade de São Paulo, São Paulo, 2020.

Com motivação no campo da Teoria Geométrica da Medida, o objetivo neste trabalho é fazer um estudo da teoria de diferenciação de medidas em espaços métricos, discutindo primeiramente teoremas de coberturas relevantes para a referida teoria, com particular destaque para o teorema de cobertura de Besicovitch-Federer em espaços métricos direcionalmente limitados. Daí, para uma certa classe de medidas borelianas em espaços métricos, são investigadas propriedades de diferenciabilidade de uma medida em relação a outra, bem como versões abstratas do teorema fundamental do cálculo nesse contexto. Por último, como aplicação desta teoria, apresentaremos a fórmula da área para aplicações contínuas entre espaços métricos, sob suposições de regularidade mínima.

Palavras-chave: Teorema de cobertura de Besicovitch, fórmula da área, teoremas de cobertura, diferenciação de medidas em espaços métricos. 


\section{Abstract}

CABRERA, J. D. C. Differentiation of Measure in Metric Spaces. 2020. 63 f. Thesis (Master) - Instituto de Matemática e Estatística, Universidade de São Paulo, São Paulo, 2020.

With motivation in the field of Geometric Theory of Measure, the objective in this work is to study the theory of differentiation of measures in metric spaces. We investigate some covering theorems related to that theory, in particular the Besicovitch-Federer's covering theorem on directionally limited metric spaces. For a certain class of Borelian measures on metric spaces, we study the notion of differentiability of a measure with respect to another, as well as abstract versions of the fundamental theorem of calculus in this context. Finally, as an application of this theory, we present the Area Formula for continuous applications between metric spaces, under assumptions of minimal regularity.

Keywords: Besicovitch's covering Theorem, Area formula, Covering's Theorem, Differentiation of Measure, Metric Spaces. 


\section{Sumário}

$\begin{array}{lll}\text { Lista de Símbolos } & \text { ix }\end{array}$

Introdução

1 Conceitos Básicos $\quad 1$

1.1 Medidas . . . . . . . . . . . . . . . . . . . . . . . 1

1.2 Funções mensuráveis . . . . . . . . . . . . . . . . . . . . . . . . . . 5

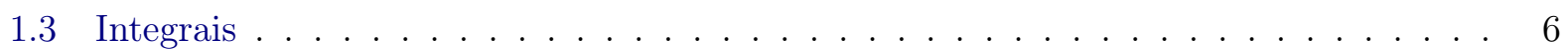

1.4 Construção de Caratheodory . . . . . . . . . . . . . . . . . . . 7

2 Teoremas de Cobertura $\quad 11$

2.1 Famílias adequadas . . . . . . . . . . . . . . . . . . . . . . 11

2.2 Espaços métricos direcionalmente limitados . . . . . . . . . . . . . . . . 15

2.3 Teorema de Cobertura de Besicovitch-Federer . . . . . . . . . . . . . . . . . 22

2.4 Relações de cobertura . . . . . . . . . . . . . . . . . . . . . . 25

3 Diferenciação de medidas $\quad 31$

3.1 Derivadas . . . . . . . . . . . . . . . . . . . . . . . 31

3.2 Densidade e Continuidade aproximada . . . . . . . . . . . . . . . . . . . 40

3.3 Derivação de medidas usando bolas centralizadas . . . . . . . . . . . . . . . . . 43

3.4 Fórmula da área em espaços métricos . . . . . . . . . . . . . . . . . . . . . . 45

$\begin{array}{ll}\text { Referências Bibliográficas } & 47\end{array}$ 


\section{Lista de Símbolos}

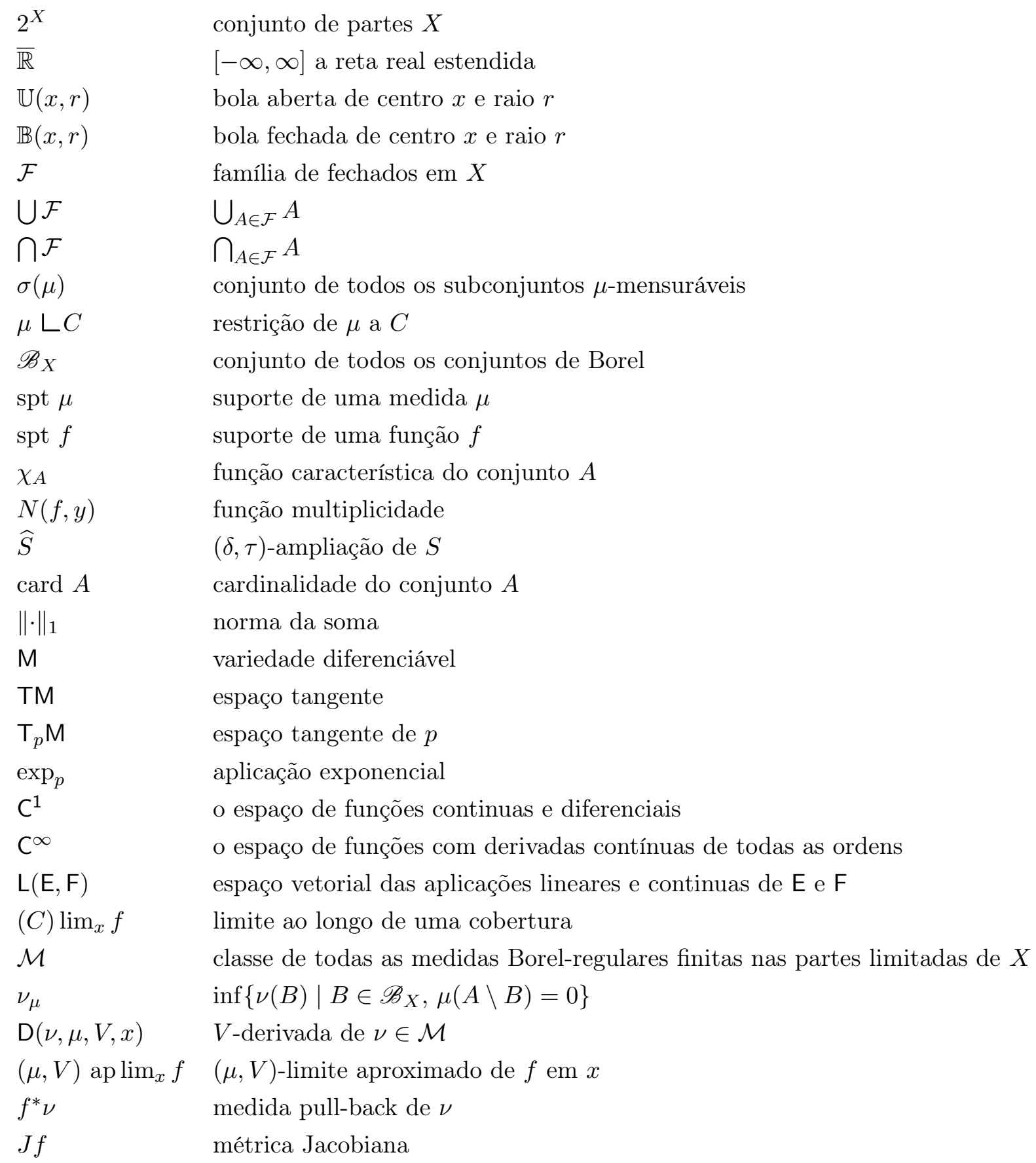




\section{Introdução}

A Teoria Geométrica da Medida estuda a estrutura e as propriedades geométricas dos conjuntos através da Teoria da Medida. Esta teoria permite abordar questões em áreas muito diferentes da matemática, incluindo-se o cálculo de variações, análise geométrica e análise harmônica [Sil11, Dom14, Tor19]. A principal referência para os leitores que desejarem iniciar seus estudos na referida teoria é o texto enciclopédico de H. Fededer [Fed96], cujo título deu origem ao nome da área.

O objetivo neste trabalho é fazer um estudo da teoria de diferenciação de medidas em espaços métricos, a qual tem origem nas obras de Besicovitch em [Bes45] e [Bes46]. Tais trabalhos, assim como seus desenvolvimentos subsequentes (vide apanhado histórico em [Rig18], bem como referências lá citadas), usam como ferramentas fundamentais certos teoremas de coberturas, o mais notável dos quais leva o nome de Besicovitch, bem como o de Federer numa versão mais abstrata a ser apresentada no capítulo 2. Em particular, a compreensão e o detalhamento das ideias contidas em [Fed96], na seção 2.8 para teoremas de cobertura e na seção 2.9 para os teoremas de diferenciação, fizeram parte importante das motivações para o desenvolvimento do presente trabalho.

A seguir, apresentamos um breve resumo dos tópicos abordados em cada capítulo desta dissertação.

No capítulo 1, faremos uma revisão dos conceitos, terminologia e resultados básicos na teoria da medida que serão usados ao longo deste trabalho. Começando com as propriedades gerais de medidas sobre um espaço métrico $(X, d)$, seguimos com alguns teoremas sobre funções mensuráveis e integração em espaços de medida, finalizando com a chamada construção de Carathéodory, a qual é uma ferramenta importante para a construção de medidas borelianas regulares em espaços métricos.

No capítulo 2, serão discutidos os teoremas de coberturas relevantes para a teoria de diferenciação de medidas. Será introduzida, em particular, a noção de espaço métrico direcionalmente limitado, proposta por Federer, a qual pretendemos apresentar de forma intuitiva e geométrica, de modo a detalhar as ideias implícitas em [Fed96]. Em tais espaços, será enunciada uma generalização do clássico teorema de cobertura de Besicovitch em $\mathbb{R}^{n}$ (cf. [EG91]), a saber, o teorema de Besicovitch-Federer, que desempenha um papel central na teoria de diferenciação de medidas borelianas regulares com propriedades de finitude adequadas.

O capítulo 3 é dedicado à teoria da diferenciação de medidas em espaços métricos. Aplicando os teoremas vistos no capítulo anterior para uma certa classe de medidas de Borel em espaços métricos, são investigados teoremas básicos de diferenciação e propriedades de diferenciabilidade de uma medida em relação a outra, bem como versões abstratas do Teorema Fundamental do Cálculo 
nesse contexto. A seguir, são introduzidas noções de "densidades de medidas", através das quais definiremos modificações dos conceitos de limite e continuidade clássicos, o que resulta em noções correspondentes no âmbito da teoria da medida mais adequadas para se tratar problemas nesta teoria. Por último, como uma aplicação dessa teoria de diferenciação, apresentaremos a fórmula de área, que generaliza o teorema de mudança de variáveis clássico (i.e. no contexto de integrais múltiplas do Cálculo Avançado) a uma versão abstrata para aplicações contínuas entre espaços métricos sob suposições de regularidade mínima, cf. [Mag11]. 


\section{Capítulo 1}

\section{Conceitos Básicos}

Neste capítulo, apresentaremos alguns conceitos teóricos gerais, terminologia, notações e resultados da Teoria da Medida que serão necessários ao longo deste trabalho. A maior parte deste material pode ser encontrada em [Fol99], [Fed96], [Mat95] e [EG91]. Os resultados que usaremos sobre espaços métricos e espaços topológicos podem ser consultados em [BI01] e [Lim70]. Muitas das provas, salvo exceções, serão omitidas.

Notação. Seja $X$ um conjunto. Dados subconjuntos $A$ e $B$ de $X$, denotaremos $A \subset B$ para dizer que $A$ está contido em $B$ ou $A=B$. Caso haja risco de confusão, será escrito $A \subseteq B$ para a inclusão não estrita e $A \subsetneq B$ para a inclusão estrita.

Seguindo 2.1.1. em [Fed96, pag. 51], cada conjunto $S \subset \overline{\mathbb{R}}$ tem um maior limite inferior inf $S$ e um menor limite superior $\sup S$. Se $S$ for não-vazio, então $\inf S \leq \sup S$. No entanto, $\sup \emptyset=-\infty$ e $\inf \emptyset=+\infty$.

Para um espaço métrico $(X, d)$, as bolas abertas e fechadas com centro $a \in X$ e raio $r>0$ são denotadas por, respectivamente,

$$
\mathbb{U}(a, r)=\{x \mid d(a, x)<r\} \quad \text { e } \quad \mathbb{B}(a, r)=\{x \mid d(a, x) \leq r\} .
$$

Para mais notações, pode-se verificar a Lista de Símbolos na página ix.

\section{$1.1 \quad$ Medidas}

Neste texto, seguindo [Fed96] e a nomenclatura tradicional em teoria geométrica da medida, usaremos o termo "medida" tanto para denotar medidas exteriores como para medidas $\sigma$-aditivas numa $\sigma$-álgebra $\mathcal{A}$ de $X$. Caso haja risco de confusão, usaremos explicitamente a nomenclatura "medida exterior" no primeiro caso.

Definição 1.1. Uma medida exterior ou, simplesmente, uma medida em um conjunto $X$ é uma função de conjunto $\mu: 2^{X} \rightarrow[0, \infty]$ tal que:

i) $\mu(\emptyset)=0$,

ii) $\mu\left(\bigcup_{n \in \mathbb{N}} A_{n}\right) \leq \sum_{n \in \mathbb{N}} \mu\left(A_{n}\right)$ (subaditividade enumerável).

Definição 1.2 (Condição de Caratheodory). Dada uma medida $\mu$ sobre $X$, um subconjunto $A \subset X$ é dito mensurável em relação a $\mu$ ou $\mu$-mensurável se satisfaz a Condição de Caratheodory:

$$
\forall T \subset X, \quad \mu(T)=\mu(T \cap A)+\mu(T \backslash A) .
$$


Definição 1.3 (Restrição de medidas). Sejam $\mu$ uma medida sobre $X$ e $C \subset X$. Definimos a restrição de $\mu$ a $C$, denotado por $\mu\llcorner C$, como

$$
\forall A \subset X, \quad \mu\llcorner C(A)=\mu(A \cap C) .
$$

Note que $\mu\llcorner C$ é uma medida em $X$ e todos os conjuntos $\mu$-mensuráveis são $\mu\llcorner C$-mensuráveis também.

Teorema 1.4 (Propriedades elementares das medidas). Sejam $\mu$ uma medida sobre $X$ e $A, B \subset X$ :

i) Se $A \subseteq B$, então $\mu(A) \leq \mu(B)$.

ii) Se A é $\mu$-mensurável, $X \backslash A$ também é $\mu$-mensurável.

iii) Se $\mathcal{F}$ é uma família enumerável não vazia de conjuntos $\mu$-mensuráveis, então $\bigcup \mathcal{F} e \bigcap \mathcal{F}$ são $\mu$-mensuráveis.

iv) Se $A_{1}, A_{2}, A_{3}, \ldots$ são conjuntos $\mu$-mensuráveis disjuntos, então

$$
\mu\left(\bigcup_{i=1}^{\infty} A_{i}\right)=\sum_{i=1}^{\infty} \mu\left(A_{i}\right) .
$$

v) (Continuidade para baixo) Se $B_{1} \subset B_{2} \subset B_{3} \subset \cdots$ formam uma sequência crescente de conjuntos $\mu$-mensuráveis, então

$$
\mu\left(\bigcup_{i=1}^{\infty} B_{i}\right)=\lim _{i \rightarrow \infty} \mu\left(B_{i}\right) .
$$

vi) (Continuidade para acima) $S e C_{1} \supset C_{2} \supset C_{3} \supset \cdots$ formam uma sequência decrescente de conjuntos $\mu$-mensuráveis, com $\mu\left(C_{1}\right)<\infty$, então

$$
\mu\left(\bigcap_{i=1}^{\infty} C_{i}\right)=\lim _{i \rightarrow \infty} \mu\left(C_{i}\right) .
$$

vii) Os conjuntos $\emptyset$ e $X$ são $\mu$-mensuráveis. Se $\mu(A)=0$, então A é $\mu$-mensurável.

viii) Se $A$ é $\mu$-mensurável e $B \subset X$, então

$$
\mu(A)+\mu(B)=\mu(A \cap B)+\mu(A \cup B) .
$$

Demonstração. Ver o Teorema 2.1.3. em [Fed96, pág. 54 e 55]. Também o Teorema 1.1 para os itens i), ii) e vii), o Teorema 1.2 para os outros itens em [EG91, pág. 2 e 3].

Definição 1.5 (Envoltório). Sejam $\mu$ uma medida sobre $X$ e $A \subset X$. Dizemos que $B \subset X$ é um $\mu$-envoltório de $A$ se for $\mu$-mensurável, $A \subset B$ e, para todo $T \subset X \mu$-mensurável,

$$
\mu(T \cap A)=\mu(T \cap B) .
$$

Observação 1.6. Note que, se $A \subset B$, com $B \mu$-mensurável e $\mu(A)=\mu(B)<\infty$, então $B$ é um $\mu$-envoltório de $A$. De fato, para todo $T \subset X \mu$-mensurável tem-se,

$$
\mu(A \cap T) \leq \mu(B \cap T) \quad \text { e } \quad \mu(A \backslash T) \leq \mu(B \backslash T) .
$$


E por outro lado, como $T$ é $\mu$-mensurável, da condição de Caratheodory temos

$$
\mu(A)=\mu(A \cap T)+\mu(A \backslash T) \quad \text { e } \quad \mu(B)=\mu(B \cap T)+\mu(B \backslash T),
$$

então, como $\mu(B)<\infty$, segue

$$
\mu(B \cap T)=\mu(B)-\mu(B \backslash T) \leq \mu(A)-\mu(A \backslash T)=\mu(A \cap T) .
$$

Portanto $\mu(A \cap T)=\mu(B \cap T)$.

Definição 1.7 (Medidas regulares). Uma medida $\mu$ sobre $X$ é chamada regular se, para cada $A \subset X$ existe um conjunto $B \mu$-mensurável tal que

$$
A \subset B \quad \text { e } \quad \mu(A)=\mu(B) .
$$

Proposição 1.8. Sejam $\mu$ uma medida regular sobre $X, A$ e $B$ subconjuntos de $X$. As seguintes afirmações são verdadeiras:

i) Para toda sequência crescente $A_{1} \subset A_{2} \subset A_{3} \subset \cdots$ de subconjuntos de $X$ (não necessariamente $\mu$-mensuráveis),

$$
\mu\left(\bigcup_{i=1}^{\infty} A_{i}\right)=\lim _{i \rightarrow \infty} \mu\left(A_{i}\right)
$$

ii) Se $\mu(A)<\infty$, então A tem um $\mu$-envoltório.

iii) Se $A \cup B$ é $\mu$-mensurável e $\mu(A)+\mu(B)=\mu(A \cup B)<\infty$, então $A$ e $B$ são $\mu$-mensuráveis.

iv) Se $C \subset X$ e $\mu(C)<\infty$, então a classe de todos os conjuntos $\mu\llcorner C$-mensuráveis é igual a

$$
\{(B \cap C) \cup S \mid S \subset X \backslash C \text { e } B \text { é } \mu \text {-mensurável }\} .
$$

Demonstração. Note que, o item ii) obtém-se da Observação 1.6. Para os outros itens, ver 2.1.5. em [Fed96, pág. 56 e 57].

Definição 1.9. Dados um conjunto $X$ e $\mathcal{A} \subset 2^{X}$, dizemos que:

i) $\mathcal{A}$ é uma álgebra de subconjuntos de $X$ se contém o conjunto vazio, é fechado sob complementação e fechado sob uniões finitas.

ii) $\mathcal{A}$ é uma $\sigma$-álgebra se é uma álgebra fechada sob uniões enumeráveis.

iii) Os conjuntos em $\mathcal{A}$ são chamados mensuráveis em relação a $\mathcal{A}$, ou $\mathcal{A}$-mensuráveis, ou (se é claro a partir do contexto) simplesmente mensuráveis.

iv) Dada uma $\sigma$-álgebra $\mathcal{A} \subset 2^{X}$, chamamos uma função $\mu: \mathcal{A} \rightarrow[0, \infty]$ de medida em $\mathcal{A}$ se $\mu(\emptyset)=0$ e, para toda sequência disjunta $\left(A_{n}\right)_{n \in \mathbb{N}}$ em $\mathcal{A}, \mu\left(\dot{\cup}_{n \in \mathbb{N}} A_{n}\right)=\sum_{n=1}^{\infty} \mu\left(A_{n}\right)$.

Definição 1.10. Uma medida $\mu: \mathcal{A} \rightarrow[0, \infty]$ é chamada:

i) completa se $E \in \mathcal{A}, \mu(E)=0$ e $A \subset E$ implica $A \in \mathcal{A}$,

ii) finita se $\mu(X)<\infty$,

iii) $\sigma$-finita se existe uma sequência $\left(E_{n}\right)_{n \in \mathbb{N}}$ em $\mathcal{A}$ tal que $\bigcup_{n \in \mathbb{N}} E_{n}=X$ e para todo $n \in \mathbb{N}$, $\mu\left(E_{n}\right)<\infty$. Mais geralmente, um conjunto $A \subset X$ é $\sigma$-finito se pode ser coberto por uma família enumerável de conjuntos mensuráveis de medida finita. 
Definição 1.11. Dado um subconjunto $S \subset 2^{X}$, definimos a $\sigma$-álgebra gerada por $S$, denotada por $\sigma(S)$, como sendo a menor $\sigma$-álgebra contendo $S$, a qual é a interseção da família de todas as $\sigma$-álgebras que contém $S$.

Definição 1.12 (Medida de Borel). Para um espaço topológico $(X, \tau)$, definimos:

i) A $\sigma$-álgebra de Borel de $X$ é a $\sigma$-álgebra gerada por $\tau$, i.e. $\sigma(\tau)$, que denotaremos por $\mathscr{B}_{X}$. Os elementos de $\mathscr{B}_{X}$ são chamados conjuntos de Borel ou simplesmente borelianos.

ii) Uma medida $\mu$ em $X$ é uma medida de Borel ou boreliana se cada boreliano for $\mu$-mensurável.

Note que, se $S \subset 2^{X}$ e $\mathcal{A}$ é a $\sigma$-álgebra de subconjuntos de $X$, então $\sigma(S) \subset \mathcal{A}$ se, e somente se, $S \subset \mathcal{A}$. Em particular, para $(X, \tau)$ um espaço topológico e uma medida $\mu$ em $X$, temos que $\mu$ é uma medida de Borel se, e somente se, cada subconjunto aberto de $X$ é $\mu$-mensurável (ou equivalentemente, se cada subconjunto fechado de $X$ é $\mu$-mensurável). No caso que a topologia seja metrizável por uma métrica $d$, um critério simples para que uma medida $\mu$ seja boreliana é dado pelo Critério de Caratheodory no Teorema 1.20.

Definição 1.13. Seja $\mu$ uma medida de Borel num espaço topológico $(X, \tau)$. Dizemos que:

i) $\mu$ é aberta $\sigma$-finita se existe uma sequência $\left(U_{n}\right)_{n \in \mathbb{N}}$ de subconjuntos abertos de $X$ tal que $X=\bigcup_{n \in \mathbb{N}} U_{n}$ e, para todo $n \in \mathbb{N}, \mu\left(U_{n}\right)<\infty$.

ii) $\mu$ é localmente finita se, para todo $x \in X$, existe uma vizinhança aberta $U$ de $x$ tal que $\mu(U)<\infty$.

Definição 1.14. Uma família $\mathcal{G}$ de subconjuntos de $X$ é uma partição boreliana de um conjunto de Borel $A$ se $\mathcal{G}$ é uma família enumerável disjunta de borelianos com $\bigcup \mathcal{G}=A$.

Definição 1.15. Seja $\mu$ uma medida num espaço topológico $(X, \tau)$.

i) Dizemos que $\mu$ é concentrada sobre um conjunto $A \subset X$ se $\mu(X \backslash A)=0$.

ii) O suporte de $\mu$ é o complemento da união de todos os conjuntos abertos $V$ em $X$ tais que $\mu(V)=0$, i.e

$$
\text { spt } \mu:=X \backslash \bigcup\{V \mid V \in \tau \text { e } \mu(V)=0\} .
$$

Na situação da definição acima, em geral não é verdade que $\mu$ seja concentrada em seu suporte, ou seja, que $\mu(X \backslash$ spt $\mu)=0$. A Proposição 1.35 em [Ter19, pág. 12] fornece duas condições suficientes para que esta propriedade seja cumprida.

Teorema 1.16 (Aproximação por conjuntos fechados e abertos). Suponhamos que $\mu$ é uma medida num espaço métrico $(X, d)$, todos os conjuntos abertos em $X$ são $\mu$-mensuráveis e $B \in \mathscr{B}_{X}$.

i) Se $\mu(B)<\infty$ e $\epsilon>0$, então $B$ contem um conjunto fechado $C$ para o qual

$$
\mu(B \backslash C)<\epsilon .
$$

ii) Se $B$ pode ser coberto por uma família enumerável de conjuntos abertos $\left(V_{i}\right)_{i \in \mathbb{N}} \operatorname{com} \mu\left(V_{i}\right)<\infty$ para todo $i \in \mathbb{N} e \epsilon>0$, então $B$ está contido num conjunto aberto $W$ para o qual

$$
\mu(W \backslash B)<\epsilon .
$$

Demonstração. Ver o Teorema 2.2.2. em [Fed96, pág. 60 e 61]. Também o Teorema 1.10 em [Mat95, pág. 11 e 12].

Definição 1.17 (Medida Borel-regular). Uma medida sobre um espaço topológico $(X, \tau)$ é chamada Borel-regular se $\mu$ é boreliana e, para cada $A \subset X$, existe $B \in \mathscr{B}_{X}$ para o qual $A \subset B$ e $\mu(A)=\mu(B)$. 
Teorema 1.18. Sejam $\mu$ uma medida Borel-regular em $X$ e $A \subset X \mu$-mensurável com $\mu(A)<\infty$. Então,

i) $\mu\llcorner A$ é Borel-regular.

ii) Existem borelianos $B$ e $D$ tais que $D \subset A \subset B$ e $\mu(B \backslash D)=0$.

Demonstração. De fato, usando a Borel regularidade de $\mu$ para $A$ existe $B \in \mathscr{B}_{X}$ tal que $A \subset B$ e $\mu(A)=\mu(B), \operatorname{logo}$ como $A$ é $\mu$-mensurável temos $\mu(B \backslash A)=0$.

1) Dado $C \subset X$, seja $D \in \mathscr{B}_{X} \operatorname{com} B \cap C \subset D$ e $\mu(B \cap C)=\mu(D)$. Então $C \subset D \cup B^{c}:=E$, e

$$
\mu\llcorner A(E)=\mu(A \cap E) \leq \mu(B \cap E)=\mu(B \cap D) \leq \mu(D)=\mu(B \cap C)=\mu(A \cap C)=\mu\llcorner A(C) .
$$

Como $C \subset E$, temos também $\mu\llcorner A(C) \leq \mu\llcorner A(E)$, de modo que $\mu\llcorner A(E)=\mu\llcorner A(C)$. Portanto $\mu\llcorner A$ é Borel-regular.

2) Pela Borel regularidade de $\mu$, para $B \backslash A$ existe $E \in \mathscr{B}_{X}, B \backslash A \subset E$ e $\mu(E)=\mu(B \backslash A)=0$. Agora, como $\left(B \cap A^{c}\right)=B \backslash A \subset E$ temos $E^{c} \subset\left(B \cap A^{c}\right)^{c}=B^{c} \cup A$, daí

$$
B \backslash E=B \cap E^{c} \subset B \cap\left(B^{c} \cup A\right)=A .
$$

Assim, temos o boreliano $D:=B \backslash E \subset A$ com,

$$
\mu(B \backslash D)=\mu(B \backslash(B \backslash E))=\mu(E)=0 .
$$

Observação 1.19. O item $i$ ) do Teorema 1.18 também é válido para qualquer medida Borel-regular em $X$ e $A$ boreliano, não necessariamente de medida finita. Além disso, do item $i i$ ) pode-se afirmar que, se $\mu$ é uma medida Borel-regular num espaço métrico, o Teorema 1.16 é válido para $B \in \sigma(\mu)$, não necessariamente boreliano.

Teorema 1.20 (Critério de Caratheodory). Uma medida $\mu$ num espaço métrico $(X, d)$ é de Borel se, e somente se,

$$
\mu(A \cup B)=\mu(A)+\mu(B)
$$

para todo $A, B \subset X$ que satisfaz $d(A, B):=\inf \{d(a, b) \mid a \in A, b \in B\}>0$.

Demonstração. Ver o Teorema 1.18 em [Ter19, pág. 6].

\subsection{Funções mensuráveis}

Agora vamos estender a noção de mensurabilidade de conjuntos para funções, considerando $X$ um conjunto não vazio, $Y$ um espaço topológico e $\mu$ uma medida em $X$.

Definição 1.21. Seja $\mu$ uma medida em $X$ e $P(X)$ uma propriedade relativa aos pontos de $X$. Diz-se que $P$ vale em quase todo $X$ com respeito à medida $\mu$ se o complemento do conjunto $\{x \in X \mid P(X)\}$ tem medida nula, i.e. $P$ vale $\mu$-q.t. $x \in X$ se $\mu(X \backslash\{x \in X \mid P(X)\})=0$. Similarmente, dizemos que:

- Se $A, B \subset X, B$ contem $\mu$-q.t. $A$ se, $\mu(A \backslash B)=0$.

- Se $f$ e $g$ duas funções com valores em $\overline{\mathbb{R}}$, então $f(x)=g(x)$ para $\mu$-q.t. $x$ se

$$
\mu(X \backslash\{x \mid f(x)=g(x)\})=0 .
$$

Neste caso, podemos usar a frase equivalente " $f$ e $g$ são $\mu$-quase sempre iguais". 
Definição 1.22 (Funções mensuráveis). Sejam $\mu$ uma medida em $X$ e $Y$ um espaço topológico. Uma função $f: \operatorname{dom} f \subset X \rightarrow Y$ é chamada mensurável em relação a $\mu$ ou $\mu$-mensurável se as seguintes condições forem válidas:

i) seu domínio cobre $\mu$-q.t. $X$, i.e. $\mu(X \backslash \operatorname{dom} f)=0$,

ii) para todo $E \subset Y$ aberto, $f^{-1}(E)$ é $\mu$-mensurável.

Definição 1.23. Seja $\mu$ uma medida sobre o espaço topológico $(X, \tau)$. Dizemos que:

i) Uma função $f: A \rightarrow \overline{\mathbb{R}}$ é uma função de Borel se $A$ é um conjunto de Borel e, para todo $c \in \mathbb{R}$, o conjunto $\{x \in A \mid f(x)<c\}$ é de Borel.

ii) Se $Y$ é um espaço topológico, a aplicação $f: X \rightarrow Y$ é uma aplicação de Borel se, para todo aberto $U \subset Y$ o conjunto $f^{-1}(U)$ é de Borel.

Notação. Seja $f$ uma função com valores em $\overline{\mathbb{R}}$. Usaremos as notações:

$$
f^{+}=\max \{f, 0\}, \quad f^{-}=\max \{-f, 0\} \quad \text { e } \quad f=f^{+}+f^{-} .
$$

Todas as propriedades das funções $\mu$-mensuráveis podem ser consultadas no 2.3.2 em [Fed96, pág. 73 e 74], também no Teorema 1.42 em [Ter19, pág. 15]. Para nosso uso, iremos destacar a seguinte propriedade:

Proposição 1.24. Sejam $X$ um conjunto e $\mu$ uma medida em $X$. Uma função $f: \operatorname{dom} f \subset X \rightarrow \overline{\mathbb{R}}$ definida $\mu$-q.s. em $X$ é $\mu$-mensurável se, e somente se, para todo $T \subset X e-\infty<a<b<\infty$,

$$
\mu(T \cap\{x \mid f(x) \leq a\})+\mu(T \cap\{x \mid f(x) \geq b\}) \leq \mu(T) .
$$

Demonstração. Ver 2.3.2 (7) em [Fed96, pág. 74 e 75].

Teorema 1.25 (Teorema de Lusin). Sejam $\mu$ uma medida Borel-regular num espaço métrico X, $f$ uma função $\mu$-mensurável com valores num espaço métrico separável $Y, A$ um conjunto $\mu$ mensurável para o qual $\mu(A)<\infty e \epsilon>0$. Então $A$ contém um conjunto fechado $C$ tal que $\mu(A \backslash C)<\epsilon$ e $\left.f\right|_{C}$ é continua.

Demonstração. Ver o Teorema 2.3.5 em [Fed96, pág. 76 e 77].

\subsection{Integrais}

Para nosso uso, mencionaremos aqui apenas algumas propriedades e observações sobre a Teoria da Integração conforme o descrito em [Fol99, Cap. 2], [Fed96, Sec. 2.4] e [Ter19, Sec 1.3].

Notação. Dados $\mu$ uma medida num conjunto $X$ e $f$ uma função $\mu$-mensurável em $X$ a valores em $[0, \infty]$, a integral de $f$ com respeito a $\mu$ é denotada por

$$
\int f d \mu \text { ou } \int f(x) d \mu(x) .
$$

Se $f$ for uma função $\mu$-mensurável em $X$ a valores em $\overline{\mathbb{R}}$, diz-se que $f$ é integrável se $\int f^{+} d \mu<\infty$ ou $\int f^{-} d \mu<\infty$; em caso afirmativo, definimos

$$
\int f d \mu:=\int f^{+} d \mu-\int f^{-} d \mu
$$

Se $\int f^{+} d \mu<\infty$ e $\int f^{-} d \mu<\infty$ (ou, equivalentemente, se $\int|f| d \mu<\infty$ ), diz-se que $f$ é somável. Alguns autores usam a nomenclatura "integrável" para o que chamamos de "somável" e "quase integrável" para o que chamamos de "integrável". 
Finalmente, se $A \in \sigma(\mu)$ e $f$ for uma função $\mu$-mensurável em $X$ a valores em $\overline{\mathbb{R}}$, definimos

$$
\int_{A} f d \mu:=\int \chi_{A} f d \mu
$$

caso $\chi_{A} f$ seja integrável, onde $\chi_{A}$ denota a função característica de $A$.

Definição 1.26. Sejam $\mu$ uma medida sobre o conjunto $X$ e $f$ uma função $X \rightarrow[0, \infty]$, não necessariamente mensurável. Definimos a integral inferior de $f$ por

$$
\int_{*} f d \mu:=\sup \left\{\int \phi d \mu \mid \phi \mu \text {-mensurável, } \operatorname{Im} \phi \subset[0, \infty) \text { enumerável, } \phi \leq f \mu \text {-q.s. }\right\},
$$

e analogamente a integral superior de $f$ como

$$
\int^{*} f d \mu:=\inf \left\{\int \psi d \mu \mid \psi \mu \text {-mensurável, } \operatorname{Im} \phi \subset[0, \infty) \text { enumerável, } f \leq \psi \mu \text {-q.s. }\right\} \text {. }
$$

Proposição 1.27. Se uma função $f: X \rightarrow[0, \infty]$ é $\mu$-mensurável, então

$$
\int f d \mu=\int_{*} f d \mu=\int^{*} f d \mu .
$$

Observação 1.28. Sejam $g$ uma função com valores em $\overline{\mathbb{R}}$ e $\mu$ uma medida no conjunto $X$. Suponha que $g(x) \geq 0$ para $\mu$-q.t. $x \in X$ e defina, para todo $A \subset X$,

$$
\nu(A):=\int_{A}^{*} g d \mu:=\int^{*} \chi_{A} g d \mu .
$$

Então $\nu$ é uma medida em $X$, e todo conjunto $\mu$-mensurável é $\nu$-mensurável. Além disso, se $\mu$ for $\sigma$-finita, $g$ é $\mu$-mensurável e: 1 ) se $\mu$ for regular, $\nu$ também o é; 2) se $X$ for um espaço topológico e $\mu$ for Borel-regular, $\nu$ também o é.

\subsection{Construção de Caratheodory}

Nesta seção, apresentaremos a construção de Caratheodory, a qual é um instrumento importante para obter medidas borelianas regulares em espaços métricos. Para mais detalhes, remetemos o leitor para o parágrafo 2.10.1 em [Fed96]. Isto será de utilidade na Seção 3.4.

Teorema 1.29 (Construção de Caratheodory). Sejam $(X, d)$ um espaço métrico, $\mathcal{F}$ uma família de subconjuntos de $X$ e $\zeta$ uma função não-negativa definida sobre $\mathcal{F}$ tal que, para todo $S \in \mathcal{F}$, $0 \leq \zeta(S) \leq \infty$. Para todo $0<\delta \leq \infty$, seja

$$
\mathcal{F}_{\delta}=\mathcal{F} \cap\{S \mid \operatorname{diam} S \leq \delta\} .
$$

Para todo $A \subset X$ definimos

$$
\mu_{\delta}(A):=\inf \left\{\sum_{S \in \mathcal{G}} \zeta(S) \mid A \subset \bigcup \mathcal{G}, \mathcal{G} \subset \mathcal{F}_{\delta} \text { enumerável }\right\} .
$$

$O$ fato de que $\mu_{\delta} \geq \mu_{\delta^{\prime}}$ para $0<\delta<\delta^{\prime} \leq \infty$ implica, para todo $A \subset X$, a existência de

$$
\nu(A)=\lim _{\delta \rightarrow 0^{+}} \mu_{\delta}(A)=\sup _{\delta>0} \mu_{\delta}(A) .
$$

Então $\mu_{\delta}$ e $\nu$ são medidas em $X$. 
Observação 1.30. Claramente $\mu_{\delta}$ e $\nu$ são medidas sobre $X$. Do Critério de Caratheodory, Teorema 1.20 , segue-se que todos os conjuntos abertos de $X$ são $\nu$-mensuráveis. De fato,

$$
\mu_{\delta}(A \cup B) \geq \mu_{\delta}(A)+\mu_{\delta}(B)
$$

sempre que $d(A, B)>\delta>0$, porque, para cada cobertura $\mathcal{G}$ de $A \cup B$ consistindo de conjuntos cujos diâmetros não excedam $\delta$, as famílias

$$
\mathcal{G}_{A}=\mathcal{G} \cap\{S \mid S \cap A \neq \emptyset\} \quad \text { e } \quad \mathcal{G}_{B}=\mathcal{G} \cap\{S \mid S \cap B \neq \emptyset\}
$$

são disjuntas e cobrem $A$ e $B$ respectivamente.

Além disso, se todos os membros de $\mathcal{F}$ são conjuntos de Borel, então cada subconjunto de $X$ está contido num conjunto de Borel com igual medida $\mu_{\delta}$ para todo $\delta>0$, e $\nu$ é uma medida Borelregular. De fato, dados $A \subset X$ e $\delta>0$, para cada $k \in \mathbb{N}$, existe $\left(B_{n, k}\right)_{n \in \mathbb{N}}$ subfamília enumerável de $\mathcal{F}_{\delta}$ (em particular, todos os $B_{n, k}$ são borelianos) com $A \subset \bigcup_{n \in \mathbb{N}} B_{n, k} \mathrm{e}$

$$
\sum_{n \in \mathbb{N}} \zeta\left(B_{n, k}\right) \leq \mu_{\delta}(A)+\frac{1}{k}
$$

Pondo $B_{\delta}:=\bigcap_{k \in \mathbb{N}}\left(\bigcup_{n \in \mathbb{N}} B_{n, k}\right)$, temos que $B_{\delta}$ é um conjunto de Borel tal que $A \subset B_{\delta}$ e, para todo $k \in \mathbb{N}, B_{\delta} \subset \bigcup_{n \in \mathbb{N}} B_{n, k}$. Logo, para todo $k \in \mathbb{N}$

$$
\mu_{\delta}\left(B_{\delta}\right) \leq \mu_{\delta}(A)+\frac{1}{k}
$$

Portanto $\mu_{\delta}\left(B_{\delta}\right) \leq \mu_{\delta}(A)$ e, como $A \subset B_{\delta}$, temos também $\mu_{\delta}(A) \leq \mu_{\delta}\left(B_{\delta}\right)$, de modo que $\mu_{\delta}\left(B_{\delta}\right)=$ $\mu_{\delta}(A)$. Finalmente, pondo $B:=\bigcap_{n \in \mathbb{N}} B_{1 / n} \in \mathscr{B}_{X}$, por monotonicidade tem-se, para todo $\delta>0$, $\mu_{\delta}(B)=\mu_{\delta}(A), \log \mathrm{o}$

$$
\nu(A)=\lim _{\delta \rightarrow 0^{+}} \mu_{\delta}(A)=\lim _{\delta \rightarrow 0^{+}} \mu_{\delta}(B)=\nu(B) .
$$

Então $\nu$ é Borel-regular, como afirmado.

Definição 1.31. Com a notação do teorema e observação acima, diremos que $\nu$ é o resultado da construção de Caratheodory com método $\zeta$ sobre $\mathcal{F}$, e nos referiremos a $\mu_{\delta}$ como a $\delta$-aproximação de $\nu$.

Exemplo 1.32 (Medidas de Hausdorff). Seja $m$ um real não negativo. Tome $\mathcal{F}=2^{X}$ e $\zeta: 2^{X} \rightarrow$ $[0, \infty]$ dada por, para todo $S \subset X$,

$$
\zeta(S):=\alpha(m) \frac{(\operatorname{diam} S)^{m}}{2^{m}}
$$

onde $\alpha(m)=\frac{\pi^{m / 2}}{\Gamma(m / 2+1)}$ (de modo a coincidir com o volume da bola unitária de $\mathbb{R}^{m}$ caso $m$ seja inteiro). O resultado da construção de Caratheodory com método $\zeta$ sobre $2^{X}$ chama-se medida de Hausdorff $m$-dimensional em $X$, denotada por $\mathcal{H}^{m}$.

Proposição 1.33 ([Fed96, 2.2.13]). Sejam $X$ um espaço métrico completo separável, $Y$ um espaço Hausdorff e $\mu$ uma medida em $Y$. Se $f: X \rightarrow Y$ é contínua e todo subconjunto fechado de $Y$ é $\mu$-mensurável, então para todo $B \in \mathscr{B}_{X}, f(B)$ é $\mu$-mensurável. 
Definição 1.34. Sejam $X$ e $Y$ espaços topológicos e a função $f: X \rightarrow Y$. A função de multiplicidade de $f, N(f, y): Y \rightarrow[0, \infty]$ é definida por

$$
N(f, y)= \begin{cases}\#\left(f^{-1}\{y\}\right) & \text { se } f^{-1}\{y\} \text { é finito } \\ \infty & \text { caso contrario }\end{cases}
$$

Teorema 1.35. Sejam $(X, d)$ um espaço métrico separável, $\nu$ uma medida sobre o espaço topológico $Y, f: X \rightarrow Y$ e suponha que para todo subconjunto de Borel $A \subset X, f(A)$ é $\nu$-mensurável. Seja, para $S \subset X, \zeta(S)=\nu(f(S))$, e $\mu$ a medida sobre $X$ obtida pela aplicaçãa da construção de Caratheodory com o método $\zeta$ na família de todos os subconjuntos de Borel em $X$, então para todo $A \subset X$ boreliano, $N\left(\left.f\right|_{A}, \cdot\right)$ é $\nu$-mensurável $e$

$$
\mu(A)=\int N\left(\left.f\right|_{A}, y\right) d \nu(y) .
$$

Demonstração. Ver Teorema 2.10.10 em [Fed96, pág 176]. 


\section{Capítulo 2}

\section{Teoremas de Cobertura}

Os teoremas de cobertura são ferramentas fundamentais em diferentes campos da Análise, usados para criar conexões entre propriedades locais e globais de medidas, e também refletem a geometria do espaço. Grosso modo, os vários teoremas de cobertura que estudaremos têm todos o mesmo objetivo qualitativo: a partir de uma cobertura arbitrária de um conjunto num espaço métrico, tenta-se extrair uma subfamília que seja, em algum sentido a ser precisado, a mais disjunta possível e que cubra quase todo o conjunto dado.

O foco do nosso estudo será dirigido a três tipos teoremas de cobertura: teoremas de cobertura para famílias adequadas em relação a uma medida, o teorema de Besicovitch-Federer e teoremas de cobertura para relações de cobertura de tipo $\mu$-Vitali, os quais serão úteis para a teoria de diferenciação de medidas que iremos desenvolver no Capítulo 3.

Salvo menção em contrário, todos os teoremas deste capítulo são devidos a Federer (cf. [Fed96, seção 2.8]).

Notação. Consideramos um espaço métrico $(X, d)$ fixo, $\mathcal{F}$ uma família de conjuntos fechados não vazios em $X$ e $\mu$ uma medida de Borel sobre $X$, a qual é finita nas partes limitadas de $X$.

\subsection{Famílias adequadas}

Definição 2.1 (Coberturas finas e adequadas). Dado $A \subset X$, diz-se que

i) $\mathcal{F}$ cobre $A$ finamente se, para todo $a \in A$, e todo $\epsilon>0$, existe $F \in \mathcal{F}$ tal que $a \in F \subset \mathbb{U}(a, \epsilon)$.

ii) $\mathcal{F}$ é $\mu$-adequada para $A$ se, para todo $U \subset X$ aberto, existe $\mathcal{G} \subset \mathcal{F}$ disjunta enumerável tal que

$$
\bigcup \mathcal{G} \subset U \quad \text { e } \quad \mu((U \cap A) \backslash \bigcup \mathcal{G})=0 .
$$

Teorema 2.2. Seja $\mathcal{K} \subset 2^{X}$ enumerável. Suponha que, para todo $A \in \mathcal{K}$, existe um número real $0<\sigma(A)<1$ tal que, para todo $W \subset X$ aberto, existe $\mathcal{H} \subset \mathcal{F}$ disjunta enumerável com

$$
\bigcup \mathcal{H} \subset W \quad \text { e } \quad \mu((W \cap A) \backslash \bigcup \mathcal{H}) \leq \sigma(A) \mu(W \cap A)
$$

Então, $\mathcal{F}$ é $\mu$-adequada para $\bigcup \mathcal{K}$.

Demonstração. É suficiente considerar o caso em que todos os elementos de $\mathcal{K}$ são limitados, portanto $\mu$-finitos. Com efeito, no caso geral podemos substituir $\mathcal{K}$ por

$$
\mathcal{K}_{b}:=\{A \cap \mathbb{U}(0, i) \mid A \in \mathcal{K}, i \in \mathbb{N}\} .
$$


Então $\mathcal{K}_{b}$ é enumerável, $\bigcup \mathcal{K}_{b}=\bigcup \mathcal{K}$ e, para todo $A_{b} \in \mathcal{K}_{b}$, escolhemos $A \in \mathcal{K}$ e $i \in \mathbb{N}$ tais que $A_{b}=A \cap \mathbb{U}(0, i)$ e definimos $\sigma_{b}\left(A_{b}\right):=\sigma(A)$. Para todo $W \subset X$ aberto, podemos aplicar a hipótese para o aberto $W \cap \mathbb{U}(0, i)$ para concluir que existe $\mathcal{H} \subset \mathcal{F}$ disjunta enumerável tal que

$$
\bigcup \mathcal{H} \subset W \cap \mathbb{U}(0, i) \quad \text { e } \quad \mu((W \cap \mathbb{U}(0, i) \cap A) \backslash \bigcup \mathcal{H}) \leq \sigma(A) \mu(W \cap \mathbb{U}(0, i) \cap A) .
$$

Portanto,

$$
\bigcup \mathcal{H} \subset W \quad \text { e } \quad \mu\left(\left(W \cap A_{b}\right) \backslash \bigcup \mathcal{H}\right) \leq \sigma_{b}\left(A_{b}\right) \mu\left(W \cap A_{b}\right),
$$

o que mostra que $\mathcal{K}_{b}$ e $\sigma_{b}\left(A_{b}\right)$ satisfazem a hipótese do teorema.

Suponhamos, pois, que todos os elementos de $\mathcal{K}$ sejam limitados. Seja $\left(B_{i}\right)_{i \in \mathbb{N}}$ uma sequência em $\mathcal{K}$ tal que, para todo $A \in \mathcal{K}, I_{A}:=\left\{i \in \mathbb{N} \mid B_{i}=A\right\}$ é infinito. Fixe $V \subset X$ aberto. Definamos indutivamente sequências $\left(W_{i}\right)_{i \geq 0}$ e $\left(\mathcal{G}_{i}\right)_{i \geq 0}$ (onde, para cada $i \geq 0, W_{i} \subset X$ aberto e $\mathcal{G}_{i} \subset \mathcal{F}$ finita disjunta) por:

1) $W_{0}:=V$ e $\mathcal{G}_{0}:=\emptyset$.

2) $W_{1}:=W_{0} \backslash \bigcup \mathcal{G}_{0}=V$. Aplique hipótese para $A=B_{1} \in \mathcal{K}$, o que fornece $0<\sigma\left(B_{1}\right)<1$ e $\mathcal{H}=\left\{\mathcal{H}_{i} \mid i \in \mathbb{N}\right\} \subset \mathcal{F}$ disjunta tal que $\bigcup \mathcal{H} \subset W_{1}$ e $\mu\left(\left(W_{1} \cap B_{1}\right) \backslash \bigcup \mathcal{H}\right) \leq \sigma\left(B_{1}\right) \mu\left(W_{1} \cap B_{1}\right)$.

a) Se $\mu\left(W_{1} \cap B_{1}\right)=0$, defina $\mathcal{G}_{1}:=\emptyset$ de modo que $\mathcal{G}_{1} \subset \mathcal{F}$ finito disjunto e

$$
\mu\left(\left(W_{1} \cap B_{1}\right) \backslash \bigcup \mathcal{G}_{1}\right)=0 \leq \sigma\left(B_{1}\right)^{\frac{1}{2}} \mu\left(W_{1} \cap B_{1}\right) .
$$

b) Se $\mu\left(W_{1} \cap B_{1}\right)>0$, então $\sigma\left(B_{1}\right) \mu\left(W_{1} \cap B_{1}\right)<\sigma\left(B_{1}\right)^{\frac{1}{2}} \mu\left(W_{1} \cap B_{1}\right)$. Como $\left\{W_{1} \backslash \bigcup_{i=1}^{n} \mathcal{H}_{i}\right\}_{n \in \mathbb{N}}$ decresce para $W_{1} \backslash \bigcup \mathcal{H}$ e como $\mu\left\llcorner B_{1}\right.$ é uma medida Boreliana finita, pela continuidade para baixo, Teorema 1.4 item $v$ ), da medida $\mu\left\llcorner B_{1}\right.$ obtém-se $n \in \mathbb{N}$ suficientemente grande tal que

$$
\mu\left(\left(W_{1} \cap B_{1}\right) \backslash \bigcup_{i=1}^{n} \mathcal{H}_{i}\right)<\sigma\left(B_{1}\right)^{\frac{1}{2}} \mu\left(W_{1} \cap B_{1}\right) .
$$

Defina $\mathcal{G}_{1}:=\left\{\mathcal{H}_{i} \mid 1 \leq i \leq n\right\}$

Em qualquer dos dois casos, obtém-se $\mathcal{G}_{1} \subset \mathcal{F}$ finita disjunta tal que

$$
\bigcup \mathcal{G}_{1} \subset W_{1} \quad \text { e } \quad \mu\left(\left(W_{1} \cap B_{1}\right) \backslash \bigcup \mathcal{G}_{1}\right)<\sigma\left(B_{1}\right)^{\frac{1}{2}} \mu\left(W_{1} \cap B_{1}\right) .
$$

3) Dado $n \in \mathbb{N}$, suponhamos $\left(W_{i}\right)_{1 \leq i \leq n}$ e $\left(\mathcal{G}_{i}\right)_{1 \leq i \leq n}$ tais que, para $1 \leq i \leq n$,

- $\mathcal{G}_{i} \subset \mathcal{F}$ finita disjunta com $\bigcup \mathcal{G}_{i} \subset W_{i}$;

- $W_{i}=W_{i-1} \backslash \bigcup \mathcal{G}_{i-1}$ (portanto, $W_{i}$ é aberto em $X$ );

- $\mu\left(\left(W_{i} \cap B_{i}\right) \backslash \bigcup \mathcal{G}_{i}\right)<\sigma\left(B_{i}\right)^{\frac{1}{2}} \mu\left(W_{i} \cap B_{i}\right)$.

Definimos $W_{n+1}:=W_{n} \backslash \bigcup \mathcal{G}_{n}$ e, para definir $\mathcal{G}_{n+1}$, reaplicamos o argumento do passo 2) com $W_{n+1}$ no lugar de $W_{1}$ e $B_{n+1}$ no lugar de $B_{1}$.

Note que $\left(W_{i}\right)_{i \in \mathbb{N}}$ é uma sequência decrescente de abertos de $V$ e, se $1 \leq i<j, \mathcal{G}_{i}$ é disjunta de $\mathcal{G}_{j}$, pois $\bigcup \mathcal{G}_{j} \subset W_{j} \subset W_{i+1}=W_{i} \backslash \bigcup \mathcal{G}_{i}$. Portanto, $\mathcal{G}:=\bigcup_{i \in \mathbb{N}} \mathcal{G}_{i} \subset \mathcal{F}$ é enumerável e disjunta, e $\bigcup \mathcal{G}=\bigcup_{i \in \mathbb{N}}\left(\bigcup \mathcal{G}_{i}\right) \subset W_{1}=V$. Além disso, para todo $i \in \mathbb{N}$, tem-se $\bigcup \mathcal{G}_{i}=W_{i} \backslash W_{i+1}$, donde

$$
V \backslash \bigcap_{i \in \mathbb{N}} W_{i}=\bigcup_{i \in \mathbb{N}} W_{i} \backslash W_{i+1}=\bigcup_{i \in \mathbb{N}}\left(\bigcup \mathcal{G}_{i}\right)=\bigcup \mathcal{G}
$$


de modo que $V \backslash \bigcup \mathcal{G}=\bigcap_{i \in \mathbb{N}} W_{i}$. Assim, para todo $A \in \mathcal{K},(V \cap A) \backslash \bigcup \mathcal{G}=A \cap\left(\bigcap_{i \in \mathbb{N}} W_{i}\right)$, o que implica, tendo em vista que $\mu\left\llcorner A\right.$ é uma medida Boreliana finita e que $\left(W_{i}\right)_{i \in \mathbb{N}}$ é uma sequência decrescente de abertos:

$$
\mu((V \cap A) \backslash \bigcup \mathcal{G})=\lim \mu\left(A \cap W_{i}\right) .
$$

Seja $\ell:=\lim _{i \rightarrow \infty} \mu\left(A \cap W_{i}\right)<\infty$. Para todo $i \in I_{A}=\left\{i \in \mathbb{N} \mid B_{i}=A\right\}$, tem-se

$$
\begin{aligned}
\mu\left(A \cap W_{i+1}\right) & =\mu\left(B_{i} \cap W_{i+1}\right)=\mu\left(B_{i} \cap\left(W_{i} \backslash \bigcup \mathcal{G}_{i}\right)\right)=\mu\left(\left(B_{i} \cap W_{i}\right) \backslash \bigcup \mathcal{G}_{i}\right)< \\
& <\sigma\left(B_{i}\right)^{\frac{1}{2}} \mu\left(W_{i} \cap B_{i}\right)=\sigma(A)^{\frac{1}{2}} \mu\left(W_{i} \cap A\right) .
\end{aligned}
$$

Como $I_{A}$ é infinito, temos que $\left\{\mu\left(A \cap W_{i+1}\right)\right\}_{i \in I_{A}}$ e $\left\{\mu\left(A \cap W_{i}\right)\right\}_{i \in I_{A}}$ são ambas subsequências de $\left\{\mu\left(A \cap W_{i}\right)\right\}_{i \in \mathbb{N}}$, logo convergem ambas para $\ell$; daí podemos concluir que $\ell \leq \sigma(A)^{\frac{1}{2}} \ell$, o que implica $\ell=0$, pois $0<\sigma(A)<1$.

Está provado, pois, que $\mu((V \cap A) \backslash \bigcup \mathcal{G})=0$ para todo $A \in \mathcal{K}$. Como $\mathcal{K}$ é enumerável, segue

$$
\mu((V \cap \bigcup \mathcal{K}) \backslash \bigcup \mathcal{G}) \leq \sum_{A \in \mathcal{K}} \mu((V \cap A) \backslash \bigcup \mathcal{G})=0
$$

donde a tese.

Corolário 2.3. Seja $\mathcal{K} \subset 2^{X}$ enumerável. Se $\mathcal{F}$ é $\mu$-adequada para cada $A \in \mathcal{K}$, então $\mathcal{F}$ é $\mu$ adequada para $\bigcup \mathcal{K}$.

Demonstração. Para todo $A \in \mathcal{K}$, seja $0<\sigma(A)<1$ arbitrário. Como $\mathcal{F}$ é $\mu$-adequada para $A$, temos que para todo $U \subset X$ aberto, existe $\mathcal{G} \subset \mathcal{F}$ disjunta enumerável tal que

$$
\bigcup \mathcal{G} \subset U \quad \text { e } \quad \mu((U \cap A) \backslash \bigcup \mathcal{G})=0 \leq \sigma(A) \mu(U \cap A) .
$$

Então pelo Teorema $2.2, \mathcal{F}$ é $\mu$-adequada para $\bigcup \mathcal{K}$.

O seguinte teorema é uma versão abstrata do teorema de cobertura de Vitali ou "five times covering lemma" (cf. [EG91, Teorema 1.24], [Sim83, Lema 3.4]).

Teorema 2.4. Sejam $\delta$ uma função não negativa e limitada em $\mathcal{F}$ e $1<\tau<\infty$. Então $\mathcal{F}$ admite uma subfamília disjunta $\mathcal{G}$ tal que, para todo $T \in \mathcal{F}$, existe $S \in \mathcal{G}$ com

$$
T \cap S \neq \emptyset \quad \text { e } \delta(T) \leq \tau \delta(S) .
$$

Demonstração. Seja $\Omega$ a classe de todas as subfamílias disjuntas $\mathcal{H} \subset \mathcal{F}$ satisfazendo a seguinte propriedade: para todo $T \in \mathcal{F}$,

$$
\begin{aligned}
& \text { ou } T \cap S=\emptyset \text { para todo } S \in \mathcal{H}, \\
& \text { ou } T \cap S \neq \emptyset \text { e } \delta(T) \leq \tau \delta(S) \text { para algum } S \in \mathcal{H} .
\end{aligned}
$$

$\Omega$ está parcialmente ordenado pela inclusão e podemos aplicar o Lema de Zorn para concluir que $\Omega$ admite um elemento maximal $\mathcal{G}$. Seja $\mathcal{K}:=\mathcal{F} \cap\{T \mid T \cap \bigcup \mathcal{G}=\emptyset\}$. Se $\mathcal{K} \neq \emptyset$, tendo em vista que $\tau>1$ e $\sup \{\delta(T) \mid T \in \mathcal{K}\}<\infty$ (pois $\delta$ é limitada, por hipótese), poderíamos escolher um $W \in \mathcal{K}$ tal que

$$
\sup \{\delta(T) \mid T \in \mathcal{K}\} \leq \tau \delta(W) .
$$

Note que $W \notin \mathcal{G}$, pois $W \in \mathcal{G}$ contradiz a definição de $\mathcal{K}$, e daí se conclui que $\mathcal{G} \subsetneq \mathcal{G} \cup\{W\}=\widetilde{\mathcal{G}}$. Agora, para todo $T \in \mathcal{F}$, se $T \cap \bigcup \mathcal{G}=\emptyset$ e $T \cap W \neq \emptyset$, então $T \in \mathcal{K}, \operatorname{logo} \delta(T) \leq \tau \delta(W)$. Portanto $\widetilde{\mathcal{G}} \in \Omega$ contrariando a maximalidade de $\mathcal{G}$. 
Então $\mathcal{K}=\emptyset$, o que significa que, para todo $T \in \mathcal{F}, T \notin\{T \mid T \cap \bigcup \mathcal{G}=\emptyset\}$, ou seja, $T \cap \bigcup \mathcal{G} \neq \emptyset$. Como $\mathcal{G} \in \Omega$, pela definição de $\Omega$ conclui-se que existe $S \in \mathcal{G}$ tal que $T \cap S \neq \emptyset$ e $\delta(T) \leq \tau \delta(S)$.

Os corolários a seguir obtém-se como consequência do Teorema 2.4, e para seu uso como suas aplicações usaremos a definição a seguir.

Definição 2.5 (Ampliação). Seja $\delta$ uma função não negativa em $\mathcal{F}$ e $1<\tau<\infty$. Definimos, para cada membro $S \in \mathcal{F}$, a sua $(\delta, \tau)$-ampliação como

$$
\widehat{S}:=\bigcup\{T \mid T \in \mathcal{F}, T \cap S \neq \emptyset, \delta(T) \leq \tau \delta(S)\} .
$$

Corolário 2.6. Seja $\mathcal{G}$ uma subfamília disjunta de $\mathcal{F}$ satisfazendo a tese do Teorema 2.4. Então

$$
\bigcup \mathcal{F} \subset \bigcup\{\widehat{S} \mid S \in \mathcal{G}\}
$$

Corolário 2.7. Sejam $A \subset X$ e $\mathcal{G}$ uma subfamília disjunta de $\mathcal{F}$ satisfazendo a tese do Teorema 2.4. Se $\mathcal{F}$ cobre $A$ finamente e $\mathcal{H}$ é um subconjunto finito qualquer de $\mathcal{G}$, então

$$
A \backslash \bigcup \mathcal{H} \subset \bigcup\{\widehat{S} \mid S \in \mathcal{G} \backslash \mathcal{H}\}
$$

Demonstração. Note que $\bigcup \mathcal{H}$ é fechado, por ser uma união finita de fechados. Como $\mathcal{F}$ cobre $A$ finamente, cada ponto de $A \backslash \bigcup \mathcal{H}$ pertence a algum $T \in \mathcal{F}$ tal que $T \cap \bigcup \mathcal{H}=\emptyset$. Daí, pelo Teorema 2.4 existe $S \in \mathcal{G} \backslash \mathcal{H}$ tal que $T \cap S \neq \emptyset$ e $\delta(T) \leq \tau \delta(S)$, de modo que $T \subset \widehat{S}$. Portanto,

$$
A \backslash \bigcup \mathcal{H} \subset \bigcup\{\widehat{S} \mid S \in \mathcal{G} \backslash \mathcal{H}\}
$$

Teorema 2.8. Sejam $A \subset X, \delta$ uma função limitada não negativa em $\mathcal{F}, 1<\tau<\infty, 1<\lambda<\infty$. Se $\mathcal{F}$ cobre A finamente e $\mu(\widehat{S})<\lambda \mu(S)$ sempre que $S \in \mathcal{F}$ e $\widehat{S}$ é a $(\delta, \tau)$-ampliação de $S$, então, $\mathcal{F}$ é $\mu$-adequada para $A$.

Demonstração. Pelo Corolário 2.3 podemos assumir que $A$ é limitado. Para todo aberto limitado $V \subset X$ temos que

$$
\mathcal{F}^{\prime}:=\mathcal{F} \cap\{S \mid S \subset V\}
$$

cobre $V \cap A$ finamente; portanto, pelo Corolário 2.7 existe uma subfamília disjunta $\mathcal{G}^{\prime}$ de $\mathcal{F}^{\prime}$ tal que, para toda $\mathcal{H} \subset \mathcal{G}^{\prime}$ finita,

$$
(V \cap A) \backslash \bigcup \mathcal{H} \subset \bigcup\left\{\widehat{S} \mid S \in \mathcal{G}^{\prime} \backslash \mathcal{H}\right\}
$$

Note que, para todo $S \in \mathcal{G}^{\prime}, \mu(S)>0$ (pois, por hipótese, $\mu(S)>\lambda^{-1} \mu(\widehat{S})$ ). Além disso,

$$
\sum_{S \in \mathcal{G}^{\prime}} \mu(\widehat{S}) \leq \lambda \sum_{S \in \mathcal{G}^{\prime}} \mu(S) \leq \lambda \mu(V)<\infty .
$$

Conclui-se, portanto, que $\mathcal{G}^{\prime}$ é enumerável. Agora,

$$
\sum_{S \in \mathcal{G}^{\prime}} \mu(\widehat{S})=\sup \left\{\sum_{S \in \mathcal{H}} \mu(\widehat{S}) \mid \mathcal{H} \subset \mathcal{G}^{\prime} \text { finito }\right\} .
$$


Daí, para todo $\epsilon>0$, existe uma subfamília finita $\mathcal{H}^{\prime} \subset \mathcal{G}^{\prime}$ tal que

$$
\sum_{S \in \mathcal{G}^{\prime}} \mu(\widehat{S})<\sum_{S \in \mathcal{H}^{\prime}} \mu(\widehat{S})+\epsilon
$$

donde

$$
\sum_{S \in \mathcal{G}^{\prime} \backslash \mathcal{H}^{\prime}} \mu(\widehat{S})<\epsilon
$$

Logo, tendo em vista que $\mathcal{H}^{\prime}$ é finita,

$$
\mu\left((V \cap A) \backslash \bigcup \mathcal{G}^{\prime}\right) \leq \mu\left((V \cap A) \backslash \bigcup \mathcal{H}^{\prime}\right) \leq \mu\left(\bigcup\left\{\widehat{S} \mid S \in \mathcal{G}^{\prime} \backslash \mathcal{H}^{\prime}\right\}\right) \leq \sum_{S \in \mathcal{G}^{\prime} \backslash \mathcal{H}^{\prime}} \mu(\widehat{S})<\epsilon .
$$

Como $\epsilon>0$ é arbitrário, conclui-se que $\mu\left((V \cap A) \backslash \bigcup \mathcal{G}^{\prime}\right)=0$.

Agora, para $V \subset X$ um aberto arbitrário (i.e. não necessariamente limitado), podemos tomar um aberto limitado $W \subset V$ tal que $V \cap A=W \cap A$ (por exemplo, tomamos $W$ como sendo a interseç̧ão de $V$ com uma bola aberta de raio suficientemente grande que contenha o limitado $A$ ). Daí, aplicando o caso já provado para o aberto $W$, existe $\mathcal{G} \subset \mathcal{F}$ enumerável disjunta tal que $\bigcup \mathcal{G} \subset W \subset V$ e $\mu((V \cap A) \backslash \bigcup \mathcal{G})=\mu((W \cap A) \backslash \bigcup \mathcal{G})=0$.

\subsection{Espaços métricos direcionalmente limitados}

A fim de enunciar e demonstrar o Teorema da Cobertura de Besicovitch-Federer na próxima seção, introduziremos a noção de espaço métrico direcionalmente limitado, proposta por Federer no parágrafo 2.8.9. de [Fed96, pág. 145]. Tentaremos detalhar e ilustrar as ideias geométricas subjacentes (tais ideias nos parecem estar implícitas no Federer, mas o que segue é a nossa interpretação) a esta noção nas seguintes definições e exemplos.

Definição 2.9 (Ponto metricamente intermediário a dois pontos dados num espaço métrico). Sejam $(X, d)$ um espaço métrico e $a, b \in X$. Diz-se que $x \in X$ é um ponto metricamente intermediário aos pontos a e $b$ se $d(a, b)=d(a, x)+d(x, b)$.

Exemplo 2.10. Sejam dois pontos distintos $a, b \in \mathbb{R}^{2}$ com a distância euclidiana temos que $x$ é um ponto metricamente intermediário entre $a$ e $b$ se ele está em $L=\{\alpha a+\beta b \mid \alpha \geq 0, \beta \geq 0, \alpha+\beta=1\}$ como na Figura 2.1. Agora, se $\mathbb{R}^{2}$ tem a distância Manhattan (i.e. a distância induzida pela norma $\left.\|\cdot\|_{1}\right)$, o $x$ é um ponto metricamente intermediário entre $a$ e $b$ se ele está em $S=\left[a_{1}, b_{1}\right] \times\left[a_{2}, b_{2}\right]$ como na Figura 2.2.

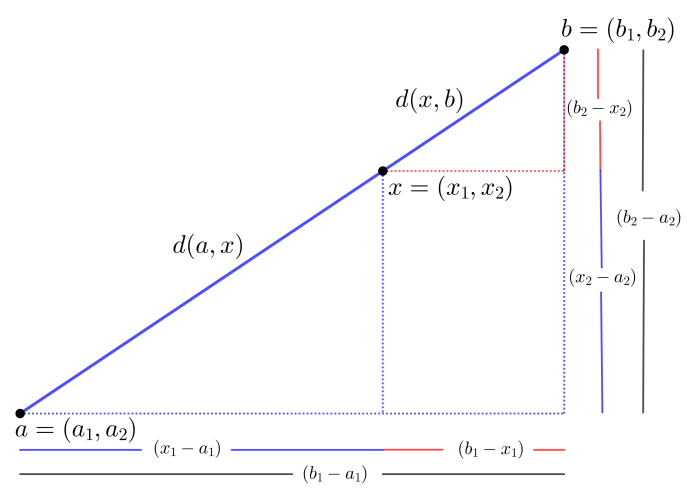

Figura 2.1: $O$ ponto $x$ metricamente intermediário entre a e $b$ dois pontos distintos em $\mathbb{R}^{2}$ com a distância euclidiana. 


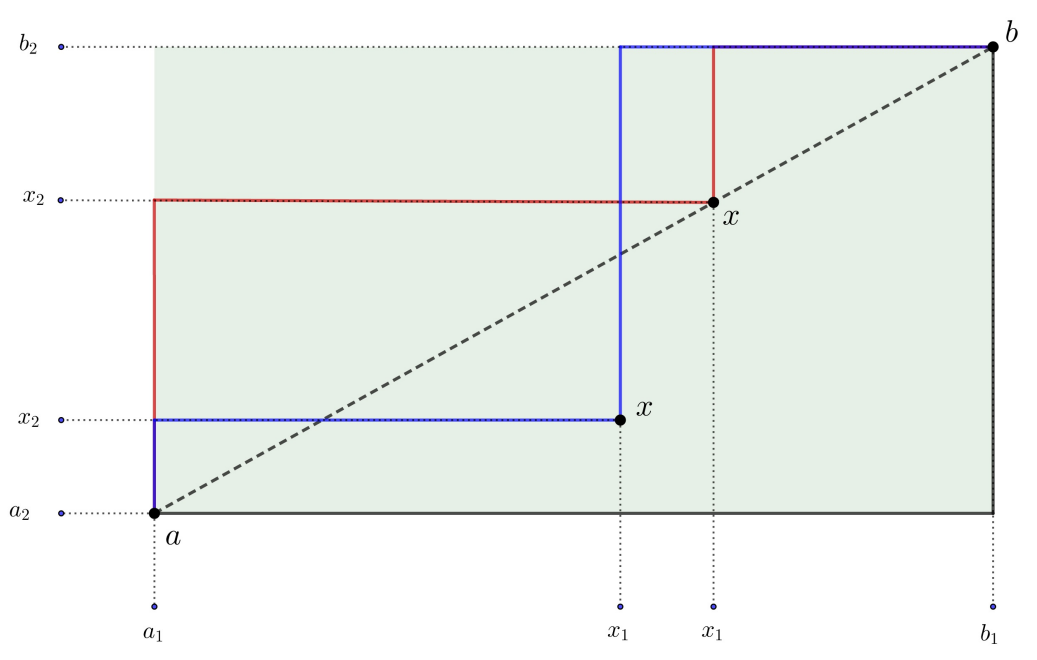

Figura 2.2: $O$ ponto $x$ metricamente intermediário entre a e $b$ dois pontos distintos em $\mathbb{R}^{2}$ com a distância Manhattan.

Definição 2.11 (Conjunto suficientemente espalhado em torno de um ponto). Sejam $(X, d)$ um espaço métrico, $a \in X, B \subset X \backslash\{a\}$ e $\eta>0$. Diz-se que $B$ é $\eta$-suficientemente espalhado em torno de $a$ se, para todos $b \neq c$ em $B$ com $d(b, a) \geq d(c, a)$, para todo $x$ metricamente intermediário a $b$ e $a$ na esfera de centro $a$ e raio $d(c, a)$, tem-se $d(x, c) \geq \eta d(c, a)$.

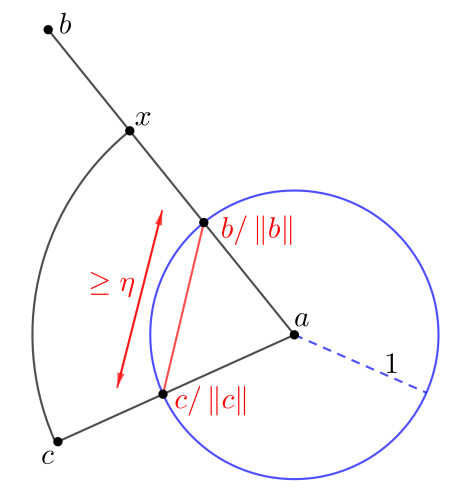

Figura 2.3: Temos $B \subset \mathbb{R}^{n} \backslash\{a\}$ conjunto suficientemente espalhado ao ponto a em $\mathbb{R}^{n}$ com a distância euclidiana.

Definição 2.12 (Métricas direcionalmente limitadas). Sejam $(X, d)$ um espaço métrico, $A \subset X$, $\xi \in(0, \infty], \eta \in\left(0, \frac{1}{3}\right]$ e $\zeta$ um inteiro positivo. Diz-se que

i) $d$ é $(\xi, \eta, \zeta)$-direcionalmente limitada em $A$ se, para todo $a \in A$ e todo $B \subset A \cap(\mathbb{U}(a, \xi) \backslash\{a\})$ $\eta$-suficientemente espalhado em torno de $a$, tem-se $\operatorname{card}(B) \leq \zeta$.

ii) $X$ é $(\xi, \eta, \zeta)$-direcionalmente limitado se $d$ for $(\xi, \eta, \zeta)$-direcionalmente limitada em $A=X$.

iii) $(X, d)$ é um espaço métrico direcionalmente limitado se for $(\xi, \eta, \zeta)$-direcionalmente limitado para algum terno $(\xi, \eta, \zeta)$. 
Seguindo [Fed96], apresentamos dois exemplos de espaços métricos direcionalmente limitados. O Exemplo 2.13, no qual $X$ é um espaço vetorial de dimensão finita, motiva o termo "direcional". O segundo exemplo 2.14, no qual $X$ é uma variedade Riemanniana (de classe maior ou igual a 2) munido da métrica intrínseca, foi o que levou a Federer a formular o conceito geral.

Em [Ito18] é provado que, no espaço euclidiano, a métrica parabólica não é direcionalmente limitada; em [BL16] são apresentados dois espaços métricos que não são direcionalmente limitados.

Exemplo 2.13 (Espaços normados de dimensão finita). Seja $(X,\|\cdot\|)$ um espaço normado de dimensão finita. Para $\xi=\infty$ e para todo $\eta \in\left(0, \frac{1}{3}\right]$, existe $\zeta$ inteiro positivo tal que $X$ é $(\xi, \eta, \zeta)$ direcionalmente limitado.

Com efeito, sejam $a \in X$ e $B \subset X \backslash\{a\} \eta$-suficientemente espalhado em torno de $a$. Fazendo uma translação da situação toda, basta considerar o caso $a=0$. Dados $b, c \in B \operatorname{com} b \neq c$ e $\|b\| \geq\|c\|$, o ponto

$$
x:=\frac{\|c\|}{\|b\|} b
$$

está na esfera de centro 0 e raio $\|c\|$ e está metricamente intermediário entre 0 e $b$. Portanto, $\|x-c\| \geq \eta\|c\|$, o que é equivalente a

$$
\left\|\frac{b}{\|b\|}-\frac{c}{\|c\|}\right\| \geq \eta
$$

Portanto, conclui-se que dois pontos distintos quaisquer $b, c$ de $B$ se projetam na esfera unitária de $(X,\|\cdot\|)$ em pontos cuja distância é maior ou igual a $\eta$.

Segue-se que a projeção radial define uma bijeção de $B$ sobre um conjunto de pontos da esfera unitária cuja distância um do outro é maior ou igual a $\eta$. Denote por $\zeta$ o supremo dos cardinais de todos os subconjuntos da esfera unitária de $(X,\|\cdot\|)$ que gozam da referida propriedade; então é claro que $\operatorname{card}(B) \leq \zeta$.

Basta verificar, pois, que $\zeta$ é finito. De fato, como a referida esfera unitária é compacta, podemos cobri-la com um número finito $N$ de abertos com diâmetro estritamente menor que $\eta$; daí $\zeta \leq N$, pois, para qualquer conjunto de pontos da esfera com cardinal maior ou igual a $N+1$, pelo princípio da casa dos pombos ao menos dois pontos de tal conjunto pertencerão ao mesmo aberto da cobertura e, portanto, a distância entre eles será estritamente menor que $\eta$.

Para o seguinte exemplo, a teoria e notação sobre variedades e métricas Riemannianas pode ser consultada em [dC15, Cap. 0 e 1]; consultar também a Lista de Símbolos (ver pág. ix).

Exemplo 2.14 (Variedades Riemannianas). Sejam (M,g) uma variedade Riemanniana e $d$ a distância riemanniana em $\mathrm{M}$. Verifiquemos que, para todo $A \subset \mathrm{M}$ compacto, $d$ é $(\xi, \eta, \zeta)$-direcionalmente limitada em $A$ se tomarmos $\xi>0$ tal que $\mathbb{U}(p, \xi)$ seja uma bola normal para todo $p \in A$ (que existe, em virtude da compacidade de $A), \eta \in\left(0, \frac{1}{3}\right]$ qualquer e $\zeta$ inteiro positivo convenientemente escolhido, conforme abaixo.

1) Existe $\lambda>0$ tal que, substituindo $\xi$ por $\xi / 2$, se necessário, $\lambda$ é constante de Lipschitz para $\exp _{p}: \mathbb{U}\left(0_{p}, \xi\right) \subset \mathrm{T}_{p} \mathrm{M} \rightarrow \mathbb{U}(p, \xi) \subset \mathrm{M}$ para todo $p \in A$.

Com efeito, como $K:=\bigcup_{p \in A} \mathbb{B}\left(0_{p}, \xi / 2\right) \subset$ dom exp $\subset$ TM é compacto e a função dom exp $\rightarrow \mathbb{R}$ dada por $v_{p} \mapsto\left\|\left(\exp _{p}\right)_{* v_{p}}\right\|_{\mathrm{L}\left(\mathrm{T}_{p} \mathrm{M}, \mathrm{T}_{\exp _{p}\left(v_{p}\right)} \mathrm{M}\right)}$ é contínua (vide a Observação 2.15 ao final da prova), tem-se

$$
\lambda:=\sup \left\{\left\|\left(\exp _{p}\right)_{*} v_{p}\right\|_{\mathrm{L}\left(\mathrm{T}_{p} \mathrm{M}, \mathrm{T}_{\exp p}\left(v_{p}\right) \mathrm{M}\right)} \mid v_{p} \in K\right\}<\infty .
$$


Afirmo que, para todo $p \in A, \lambda$ é constante de Lipschitz para

$$
\exp _{p}: \mathbb{U}\left(0_{p}, \xi / 2\right) \subset \mathrm{T}_{p} \mathrm{M} \rightarrow \mathbb{U}(p, \xi / 2) \subset \mathrm{M} .
$$

De fato, dados $p \in A, v, w \in \mathbb{U}\left(0_{p}, \xi / 2\right) \subset \mathrm{T}_{p} \mathrm{M}$ e $\gamma:[0,1] \rightarrow \mathbb{U}\left(0_{p}, \xi / 2\right)$ curva seccionalmente $\mathrm{C}^{1}$ ligando $v$ a $w$, tem-se $\Gamma:=\exp _{p} \circ \gamma$ seccionalmente $C^{1}$ ligando $\exp _{p} v$ a $\exp _{p} w$. Portanto,

$$
\begin{aligned}
d\left(\exp _{p} v, \exp _{p} w\right) & \leq \ell(\Gamma)=\int_{0}^{1}\left\|\Gamma^{\prime}(t)\right\| \mathrm{d} t \\
& =\int_{0}^{1}\left\|\left(\exp _{p}\right)_{* \gamma(t)} \cdot \gamma^{\prime}(t)\right\| \mathrm{d} t \\
& \leq \lambda \int_{0}^{1}\left\|\gamma^{\prime}(t)\right\| \mathrm{d} t=\lambda \ell(\gamma) .
\end{aligned}
$$

Tomando-se o ínfimo do segundo membro para $\gamma$ no conjunto das curvas seccionalmente $\mathrm{C}^{1}$ a valores em $\mathbb{U}\left(0_{p}, \xi / 2\right)$ ligando $v$ a $w$, conclui-se que

$$
d\left(\exp _{p} v, \exp _{p} w\right) \leq \lambda\|v-w\|_{\mathrm{T}_{p} \mathrm{M}}
$$

o que prova a afirmação.

2) Sejam $a \in A$ e $B \subset \mathbb{U}(a, \xi) \backslash\{a\} \eta$-suficientemente espalhado em torno de $a$. Provemos que card $(B) \leq \zeta$, onde $\zeta$ é a cardinalidade máxima de um subconjunto da esfera unitária do $\mathbb{R}^{n}$ (munido da norma euclidiana) cujos pontos distam entre si $\frac{\eta}{\lambda}$ ou mais; conforme visto no exemplo anterior, $\zeta$ é um inteiro positivo.

Com efeito, dados $b \neq c \in B$ com $d(b, a) \geq d(c, a)$, seja $x$ o ponto obtido pela intersecção da esfera geodésica de centro $a$ e raio $d(a, c)$ com o traço da geodésica radial de $a$ até $c$ (a qual é minimizante, pois $\mathbb{U}(a, \xi) \subset \mathrm{M}$ é uma bola normal). Então $x$ está metricamente intermediário entre $a$ e $b$, de modo que, pela hipótese sobre $B, d(x, c) \geq \eta d(a, c)$. Denotando com um "til" as imagens dos pontos $a, b, c, x$ em $\mathbb{U}\left(0_{a}, \xi\right) \subset \mathrm{T}_{a} \mathrm{M}$ por $\exp _{a}^{-1}, \tilde{x}$ é um ponto na intersecção do segmento $\left[\tilde{a}=0_{a}, \tilde{b}\right]$ com a esfera de centro $0_{a}$ e raio $d(a, c)=\|\tilde{c}\|_{\mathrm{T}_{a} \mathrm{M}}$ de $\left(\mathrm{T}_{a} \mathrm{M}, \mathrm{g}_{a}\right)$; em vista do item anterior, tem-se

$$
\|\tilde{x}-\tilde{c}\|_{\mathrm{T}_{a} \mathrm{M}} \geq \frac{1}{\lambda} d(x, c) \geq \frac{\eta}{\lambda} d(a, c)=\frac{\eta}{\lambda}\|\tilde{c}\|_{\mathrm{T}_{a} \mathrm{M}} .
$$

Daí se conclui que

$$
\|\underbrace{\| \frac{\tilde{x}}{\|\tilde{x}\|}}_{=\frac{\tilde{b}}{\|\tilde{b}\|}}-\frac{\tilde{c}}{\|\tilde{c}\|}\|_{\mathrm{T}_{a} \mathrm{M}} \geq \frac{\eta}{\lambda} .
$$

Portanto, a projeção radial de $\mathrm{T}_{a} \mathrm{M}$ composta com $\exp _{a}^{-1}$ leva $B$ bijetivamente sobre um conjunto da esfera unitária de $\left(\mathrm{T}_{a} \mathrm{M}, \mathrm{g}_{a}\right)$ cujos pontos distam entre si $\frac{\eta}{\lambda}$ ou mais. Como $\left(\mathrm{T}_{a} \mathrm{M}, \mathrm{g}_{a}\right)$ é linearmente isométrico ao $\mathbb{R}^{n}$ com a norma euclidiana, segue-se que o cardinal de um tal conjunto é menor ou igual a $\zeta$, i.e. card $(B) \leq \zeta$, como afirmado.

Observação 2.15. Sejam $M$ variedade diferenciável e $E, F$ fibrados riemannianos sobre $M$. Então a aplicação $\|\cdot\|: \mathrm{L}(\mathrm{E}, \mathrm{F}) \rightarrow \mathbb{R}$ dada por $T \in \mathrm{L}\left(\mathrm{E}_{q}, \mathrm{~F}_{q}\right) \mapsto\|T\|_{\mathrm{L}\left(\mathrm{E}_{q}, \mathrm{~F}_{q}\right)}$ (i.e. associa cada $T$ à sua norma de operador) é contínua.

Com efeito, em alguma vizinhança $U$ de cada ponto de $\mathrm{M}$ podemos trivializar $\mathrm{E}$ e $\mathrm{F}$ por referenciais ortonormais $\mathcal{E}:=\left(E_{1}, \ldots, E_{n}\right)$ e $\mathcal{F}:=\left(F_{1}, \ldots, F_{m}\right)$, com respectivos correferenciais duais $\left(E^{1}, \ldots, E^{n}\right)$ e $\left(F^{1}, \ldots, F^{m}\right)$. Então $\left(E^{i} \otimes F_{j}\right)_{i, j}$ pode ser usado para trivializar $\left.\mathrm{L}(\mathrm{E}, \mathrm{F})\right|_{U} \equiv$ $U \times \mathrm{L}\left(\mathbb{R}^{n}, \mathbb{R}^{m}\right)$; como as trivializações por $\mathcal{E}$ e $\mathcal{F}$ são isometrias lineares nas fibras, o representante 
de $\|\cdot\|$ na trivialização acima é dado pela projeção no segundo fator seguida pela norma de operador de $\mathrm{L}\left(\mathbb{R}^{n}, \mathbb{R}^{m}\right)$, a qual é contínua.

Para mostrar que, na prova do teorema acima, a função dom exp $\rightarrow \mathbb{R}$ dada por

$$
v_{p} \mapsto\left\|\left(\exp _{p}\right)_{* v_{p}}\right\|_{\mathrm{L}\left(\mathrm{T}_{p} \mathrm{M}, \mathrm{T}_{\exp _{p}\left(v_{p}\right)} \mathrm{M}\right)}
$$

é contínua, tomamos $\mathcal{U}:=\operatorname{dom} \exp \left(\right.$ um aberto de $\mathrm{TM}$ contendo a seção nula), $\mathrm{E}:=\left.\mathrm{VTM}\right|_{\mathcal{U}}$ (i.e. a restrição do fibrado vertical de $\tau_{\mathrm{M}}: \mathrm{TM} \rightarrow \mathrm{M}$ a $\mathcal{U}$ ) e $\mathrm{F}:=\exp ^{*} \mathrm{TM}$ (i.e. o fibrado pullback de TM pela exponencial exp $: \mathcal{U} \subset \mathrm{TM} \rightarrow \mathrm{M}$ ), os quais são fibrados riemannianos com as métricas induzidas pelo tensor métrico g de $M$. A função em questão é obtida pela composição de $\|\cdot\|: \mathrm{L}(\mathrm{E}, \mathrm{F}) \rightarrow \mathbb{R}$ (a qual é contínua, como vimos acima) com a aplicação $\mathrm{C}^{\infty} \mathcal{U} \rightarrow \mathrm{L}(\mathrm{E}, \mathrm{F})$ dada por $v_{p} \mapsto\left(\exp _{p}\right)_{* v_{p}} \in \mathrm{L}\left(\mathrm{T}_{v_{p}}\left(\mathrm{~T}_{p} \mathrm{M}\right), \mathrm{T}_{\exp _{p}\left(v_{p}\right)} \mathrm{M}\right) \equiv \mathrm{L}(\mathrm{E}, \mathrm{F})_{v_{p}}$.

Como a discussão sobre famílias de bolas fechadas envolve uma referência constante a centros e raios, e como nem o centro nem o raio de uma bola precisam ser únicos, provaremos certas proposições sobre os subconjuntos $P \subset X \times\{r \mid 0<r<\infty\}$ e, posteriormente, associando-se a cada par $(a, r) \in P$ a bola fechada $\mathbb{B}(a, r)$, serão obtidos resultados sobre suas famílias correspondentes $\{\mathbb{B}(a, r) \mid(a, r) \in P\}$.

Definição 2.16 (Conjunto $\tau$-controlado). Sejam $(X, d)$ um espaço métrico, $P \subset X \times(0, \infty)$ e $1<\tau<\infty$. Diz-se que $P$ é $\tau$-controlado se, para todos $(a, r),(b, s)$ distintos em $P$,

$$
d(a, b)>r>s / \tau \quad \text { ou } \quad d(a, b)>s>r / \tau .
$$

Em particular, com a condição acima, tem-se $a \neq b$ e $\mathbb{B}(a, r) \neq \mathbb{B}(b, s)$, pois $b \notin \mathbb{B}(a, r)$ ou $a \notin \mathbb{B}(b, r)$.

Temos uma vaga intuição geométrica sobre o significado da definição acima, mas não temos clareza sobre qual foi a ideia que motivou Federer. Eis o que interpretamos: associando-se a cada par $(a, r) \in P$ a bola fechada $\mathbb{B}(a, r)$, a ideia é que as tais bolas fiquem "suficientemente separadas", de modo a serem verificadas as observações abaixo:

1. Duas tais bolas, digamos, associadas as pares $(a, r)$ e $(b, s)$, podem se intersectar, mas a intersecção não pode conter ambos os centros $a$ e $b$. Isso decorre da primeira desigualdade na definição, i.e. $d(a, b)>r$ ou $d(a, b)>s$.

2. A segunda desigualdade, i.e. $r>s / \tau$ ou $s>r / \tau$, controla, de certa forma, o tamanho da intersecção das bolas da família. Por exemplo, com esta condição, garante-se a existência de um $0<k<1$ tal que, se multiplicarmos os raios de todas as bolas por esta mesma contante $k$, as bolas ficam duas a duas disjuntas; $k=\frac{1}{1+\tau}$ funciona para isso.

Lema 2.17. Sejam $(X, d)$ um espaço métrico, $1<\tau<\infty, 0<\lambda<\infty$ e $Q \subset X \times(0, \lambda)$ não vazio. Então $Q$ possui um subconjunto $R$ tal que:

i) $S e(a, r),(b, s) \in R$ são distintos, então $d(a, b)>r+s$.

ii) Para todo $(a, r) \in Q$, existe $(b, s) \in R$ tal que $d(a, b) \leq r+s$ e $s>r / \tau$.

Pensando na interpretação dos pares ordenados em $X \times(0, \lambda)$ em termos de bolas fechadas, o lema acima é uma versão abstrata do Teorema 2.4, ou seja, uma abstração do teorema de cobertura de Vitali no caso em que $\mathcal{F}$ é uma família de bolas fechadas e a função $\delta$ daquele teorema é o raio das bolas (num espaço métrico em que o raio das bolas não fique intrinsecamente determinado, $\delta$ é uma escolha do raio para cada bola da família $\mathcal{F}$ ). 
Demonstração. Consideremos $\Omega$ a classe de todos os subconjuntos $R$ de $Q$ que satisfazem $i$ ) assim como a seguinte condição:

iii) Para todo $(a, r) \in Q$

$$
\begin{aligned}
& \text { ou } d(a, b)>r+s, \text { para todo }(b, s) \in R, \\
& \text { ou } d(a, b) \leq r+s \text { e } s>r / \tau \text { para algum }(b, s) \in R .
\end{aligned}
$$

Temos que $\Omega$ é diferente de vazio (pois $\emptyset \in \Omega$ ) e está ordenado parcialmente pela inclusão. Dado $T \subset \Omega$ totalmente ordenado, $R:=\bigcup T \in \Omega$ é um limitante superior para $T$. Podemos, pois, aplicar o Lema de Zorn para concluir que $\Omega$ admite um elemento maximal.

Supondo que $R$ seja um tal elemento maximal em $\Omega$ e não satisfaça $i i)$, a condição iii) implica que existe $(a, r) \in Q$ tal que, para todo $(b, s) \in R, d(a, b)>r+s$. Ou seja, o conjunto

$$
K:=Q \cap\{(a, r) \mid d(a, b)>r+s, \text { para todo }(b, s) \in R\}
$$

é não vazio. É possível tomar $(c, t) \in K \operatorname{com} \tau t>\sup \{r \mid(a, r) \in K\}$ porque $\tau>1$ e o sup é finito (pois $\lambda<\infty)$. Note que $(c, t) \notin R$, caso contrário obtemos uma contradição com $(b, s)=(c, t)$, i.e. $0=d(c, c)>2 t$.

Seja $\widetilde{R}:=R \cup\{(c, t)\} \supsetneq R$. Provemos que $\widetilde{R} \in \Omega$. De fato, é claro que $\widetilde{R}$ satisfaz $i)$. Agora, dado $(a, r) \in Q$, se não valer a primeira condição de iii $)$ para $\widetilde{R}$, existe $(b, s) \in \widetilde{R}$ tal que $d(a, b) \leq r+s$. Se $(b, s) \in R$, então vale a segunda condição de $i i i)$ porque $R \in \Omega$. Se, por outro lado $d(a, b)>r+s$ para todo $(b, s) \in R$ e $d(a, c) \leq r+t$, então $(a, r) \in K$, $\log _{\widetilde{R}} r<\tau t$ pela escolha de $(c, t)$, portanto vale a segunda condição de $i i i) \operatorname{com}(b, s)=(c, t)$. Então $\widetilde{R} \in \Omega$ contrariando a maximalidade de $R$. Portanto, qualquer elemento maximal $R$ de $\Omega$ deve satisfazer a condição $i i$ ).

Teorema 2.18. Sejam $(X, d)$ um espaço métrico e $A \subset X$. Suponha que d seja $(\xi, \eta, \zeta)$-direcionalmente limitada em $A$ e que $\tau \in \mathbb{R}$ satisfaça

$$
1<\tau<2-\eta, \quad \eta+\frac{\tau}{2-\eta}+\tau(\tau-1)<1 .
$$

Seja $P$ um subconjunto $\tau$-controlado de $A \times(0, \infty)$. Se existir $(a, r) \in P$ tal que, para todo $(b, s) \in P$,

$$
d(a, b)<\xi, \quad d(a, b) \leq r+s \quad e \quad s>r / \tau,
$$

então card $P \leq 2 \zeta+1$.

Note que, como $0<\eta \leq \frac{1}{3}$, temos que $\eta+\frac{1}{2-\eta} \leq \frac{1}{3}+\frac{3}{5}<1$. Assim, qualquer número um pouco maior que 1 é uma escolha permissível para $\tau$.

Demonstração. Suponha que exista $(a, r) \in P$ satisfazendo a hipótese. Com $k=\frac{2-\eta}{\tau}>1$, definamos

$$
\begin{aligned}
& P_{1}:=P \cap\{(b, s) \mid 0<d(a, b) \leq k r\} \\
& P_{2}:=P \cap\{(b, s) \mid d(a, b)>k r\} .
\end{aligned}
$$

Como $P$ é $\tau$-controlado, para $j=1,2$ a projeção no primeiro fator induz uma correspondência um a um entre $P_{j}$ e o conjunto

$$
B_{j}=\left\{b \mid(b, s) \in P_{j} \text { para algum } s\right\} .
$$

Verifiquemos que $B_{j}$ é $\eta$-suficientemente espalhado em torno de $a$, no sentido da Definição 2.11 . Para todo $(b, s)$ e $(c, t)$ membros distintos de $P_{j}$, com $d(a, b) \geq d(a, c)$ e $x \in X$ metricamente 
intermediário entre $b$ e $a$ (cf. 2.9) na esfera de centro $a$ e raio $d(a, c)$, tem-se:

$$
d(b, c) \leq d(b, x)+d(x, c)=d(a, b)-d(a, x)+d(x, c)=d(a, b)-d(a, c)+d(x, c) .
$$

Portanto,

$$
d(x, c) \geq d(a, c)+d(b, c)-d(a, b) .
$$

1) No caso $j=1$, usamos as desigualdades

$$
\begin{array}{llrl}
d(b, c)>\inf \{s, t\}>r / \tau, & & k r \geq d(a, b), \\
d(a, c)>\inf \{r, t\}>r / \tau, & & k \tau-1=1-\eta>0 .
\end{array}
$$

Daí,

$$
\begin{aligned}
d(x, c) & >d(a, c)+\frac{r}{\tau}-k r=d(a, c)-\frac{r}{\tau}(k \tau-1)=d(a, c)-\frac{r}{\tau}(1-\eta)> \\
& >d(a, c)(1-(1-\eta))=d(a, c) \eta
\end{aligned}
$$

2) Para o caso $j=2$, pela hipótese e pela definição de $P_{2}$, temos $d(a, b) \leq r+s, d(a, c)>k r$, bem como

$$
\begin{array}{lllll}
d(b, c)>s & \text { ou } & d(b, c)>t>s / \tau, & \text { portanto } & d(b, c)-s>t-\tau t \\
d(a, c)>t & \text { ou } & d(a, c)>r>t / \tau, & \text { portanto } & d(a, c)>t / \tau .
\end{array}
$$

Assim,

$$
\begin{aligned}
d(x, c) & \stackrel{(2.2)}{\geq} d(a, c)+d(b, c)-d(a, b) \geq \\
& \geq d(a, c)+d(b, c)-(r+s)=(d(a, c)-r)+(d(b, c)-s)> \\
& >d(a, c)-r+t(1-\tau)=d(a, c)-r-t(\tau-1)^{-r>-k^{-1} d(a, c),-t>-\tau d(a, c)} \\
& >d(a, c)-k^{-1} d(a, c)-\tau d(a, c)(\tau-1)= \\
& =d(a, c)\left(1-k^{-1}-\tau(\tau-1)\right)=d(a, c)\left(1-\frac{\tau}{2-\eta}-\tau(\tau-1)\right)> \\
& >d(a, c) \eta
\end{aligned}
$$

Portanto, em qualquer dos casos $j=1$ ou $j=2$, temos $d(x, c) \geq \eta d(a, c)$, provando que $B_{j}$ é $\eta$-suficientemente espalhado em torno de $a$, como afirmado. Finalmente, como a métrica $d$ é $(\xi, \eta, \zeta)$-direcionalmente limitada em $A$ e $B_{j} \subset A \cap \mathbb{U}(a, \xi) \backslash\{a\}$, segue card $P_{j}=$ card $B_{j} \leq \zeta$. Portanto, como $P=P_{1} \cup P_{2} \cup\{a\}$, conclui-se que card $P \leq 2 \zeta+1$.

Corolário 2.19. Sejam $(X, d)$ um espaço métrico e $A \subset X$. Suponha que d seja $(\xi, \eta, \zeta)$-direcionalmente limitada em $A$ e que $\tau \in \mathbb{R}$ satisfaça (2.1). Se $0<\lambda<\xi / 2$ e $Q$ é um subconjunto $\tau$-controlado de $A \times\{r \mid 0<r<\lambda\}$, então a família de bolas fechadas $\{\mathbb{B}(a, r) \mid(a, r) \in Q\}$ é a união de $2 \zeta+1$ subfamilias disjuntas.

Demonstração. Seja $\mathcal{G}:=\{\mathbb{B}(a, r) \mid(a, r) \in Q\}$. Sabemos que existe uma correspondência um a um entre $\mathcal{G}$ e $Q$ pelo fato de que $Q$ é $\tau$-controlado. Definiremos os subconjuntos $Q_{j}$ e $R_{j}$ de $Q$ por indução, iniciando com $Q_{0}=Q$ e $R_{0}=\emptyset$. Dado $j \geq 1$, assumindo-se os $Q_{i}$ e $R_{i}$ definidos para $i<j$, defina

$$
Q_{j}:=Q_{j-1} \backslash R_{j-1} .
$$

O Lema 2.17 garante a existência de $R_{j} \subset Q_{j}$ satisfazendo as condições:

1) Para todo $(a, r)$ e $(b, s)$ elementos distintos de $R_{j}, d(a, b)>r+s$. 
2) Para todo $(a, r) \in Q_{j}$ existe $(b, s) \in R_{j} \operatorname{com} d(a, b) \leq r+s$ e $s>r / \tau$.

Para cada $j$, da condição 1$)$ conclui-se que $\left\{\mathbb{B}(a, r) \mid(a, r) \in R_{j}\right\}$ é disjunto. Concluiremos a prova mostrando que

$$
Q_{2 \zeta+2}=\bigcap_{j=1}^{2 \zeta+1}\left(Q \backslash R_{j}\right)=Q \backslash \bigcup_{j=1}^{2 \zeta+1} R_{j}
$$

é vazio. De fato, se $(a, r) \in Q_{2 \zeta+2}$, podemos usar a condição 2) para escolher

$$
\left(b_{j}, s_{j}\right) \in R_{j} \quad \operatorname{com} \quad d(a, b) \leq r+s_{j} \quad \text { e } \quad s_{j}>r / \tau
$$

para $j=1,2, \ldots, 2 \zeta+1$. Obtemos, assim, o conjunto $\tau$-controlado

$$
P:=\{(a, r)\} \cup\left\{\left(b_{j}, s_{j}\right) \mid j=1,2, \ldots, 2 \zeta+1\right\} .
$$

Como os pares $\left(b_{j}, s_{j}\right),(a, r)$ são dois a dois distintos, temos card $P=2 \zeta+2$. No entanto, como $d\left(a, b_{j}\right) \leq r+s_{j}<2 \lambda<\xi$, o Teorema 2.18 implica que card $P \leq 2 \zeta+1$, chegando-se a uma contradição. Daí $Q_{2 \zeta+2}=\emptyset$, como afirmado. Logo, $\bigcup_{j=1}^{2 \zeta+1} R_{j}=Q$ e, portanto,

$$
\mathcal{G}=\bigcup_{j=1}^{2 \zeta+1}\left\{\mathbb{B}(a, r) \mid(a, r) \in R_{j}\right\} .
$$

\subsection{Teorema de Cobertura de Besicovitch-Federer}

Iniciamos esta seção com o clássico Teorema de Cobertura de Besicovitch, inicialmente estabelecido para o plano por Besicovitch em [Bes45] e [Bes46]. O enunciado abaixo e seu corolário são generalizações para o $\mathbb{R}^{n}$ de resultados obtidos por Besicovitch no $\mathbb{R}^{2}$ nos artigos mencionados supra. Cabe mencionar, no entanto, que os resultados originais de Besicovitch no plano parecem ser mais gerais.

Teorema 2.20 (Teorema de cobertura de Besicovitch, [EG91, Teorema 1.27]). Existe uma constante inteira positiva $N_{n}$, dependendo apenas da dimensão $n$ do espaço $X=\mathbb{R}^{n}$, tal que, se $\mathcal{F}$ for uma coleção de bolas fechadas de raios estritamente positivos com $\sup \{\operatorname{diam} B \mid B \in \mathcal{F}\}<\infty$, então o conjunto $A$ formado pelos centros das bolas de $\mathcal{F}$ pode ser coberto pela reunião de $N_{n}$ subfamílias disjuntas de $\mathcal{F}$. Ou seja, existem subfamilias disjuntas $\mathcal{G}_{1}, \ldots, \mathcal{G}_{N_{n}} \subset \mathcal{F}$ tais que

$$
A \subset \bigcup_{i=1}^{N_{n}} \bigcup_{B \in \mathcal{G}_{i}} B .
$$

Corolário 2.21 ([EG91, Teorema 1.28]). Sejam $\mu$ uma medida de Borel em $\mathbb{R}^{n}$ e $\mathcal{F}$ uma coleção de bolas fechadas de raios estritamente positivos em $\mathbb{R}^{n}$. Seja $A$ o conjunto dos centros das bolas de $\mathcal{F}$. Suponha $\mu(A)<\infty e \inf \{r \mid \mathbb{B}(a, r) \in \mathcal{F}\}=0$ para todo a $\in$ A. Então, para todo aberto $U \subset \mathbb{R}^{n}$, existe uma família enumerável $\mathcal{G}$ de bolas disjuntas de $\mathcal{F}$ tal que

$$
\bigcup \mathcal{G} \subset U \quad \text { e } \quad \mu((U \cap A) \backslash \bigcup \mathcal{G})=0 .
$$

Além da referência citada junto ao enunciado acima, o leitor interessado poderá encontrar mais detalhes e ideias sobre a geometria do Teorema de Cobertura de Besicovitch para famílias de bolas em $\mathbb{R}^{n}$ em [Hei01], [Tal13], [BL16] e [Mat95]. 
Tendo em vista o Exemplo 2.13, o teorema acima e seu corolário são casos particulares, respectivamente, do Teorema 2.22 e do Corolário 2.24, que estudaremos a seguir, devidos a Federer ([Fed96, 2.8.14, 2.8.15]), nos quais o teorema de cobertura de Besicovitch clássico é estendido a espaços métricos direcionalmente limitados. Esta versão generalizada, que chamaremos Teorema de Cobertura de Besicovitch-Federer, é sobre a geometria das bolas no espaço envolvendo a função de distância, e sua aplicabilidade é, em geral, mais ampla que a dos teoremas de cobertura vistos anteriormente na Seção 2.1 (por exemplo, é necessária alguma estrutura adicional para a " $(\delta, \tau)$-ampliação" do Teorema 2.8).

Outras versões deste teorema podem ser consultadas em [Ito18] para bolas parabólicas no espaço euclidiano, embora a métrica parabólica não seja direcionalmente limitada, e em [Rig18] onde se foca numa propriedade de cobertura chamada de Propriedade de Cobertura Fraca de Besicovitch, analisando resultados recentes sobre a validade ou não validade desta propriedade de cobertura.

Não imporemos nenhuma hipótese adicional à medida $\mu$ sobre $X$ (recorde que $\mu$ é uma medida boreliana em $X$, finita nas partes limitadas de $X$, conforme fixado no início do capítulo), mas assumiremos que $\mathcal{F}$ é uma família de bolas fechadas com raios estritamente positivos. Dado $A \subset X$, exigiremos que todos os pontos de $A$ sejam o centro (não apenas um elemento) de algum membro da família de bolas fechadas $\mathcal{F}$ e que a métrica $d$ satisfaça a condição geométrica de limitação direcional conforme a Definição 2.12 .

Teorema 2.22 (Teorema de cobertura de Besicovitch-Federer). Sejam $(X, d)$ um espaço métrico, $d(\xi, \eta, \zeta)$-direcionalmente limitada em $A \subset X, 0<\lambda<\xi / 2, \mathcal{F}$ uma familia de bolas fechadas em $X$ com raios estritamente positivos e menores que $\lambda$. Suponha que cada ponto de $A$ é o centro de algum membro de $\mathcal{F}$. Então $A$ está contido na união de $2 \zeta+1$ subfamílias disjuntas de $\mathcal{F}$.

Este teorema será demonstrado a partir do lema a seguir.

Lema 2.23. Seja $(X, d)$ um espaço métrico. Se $1<\tau<\infty, 0<\lambda<\infty$ e $P \subset X \times\{r \mid 0<r<\lambda\}$, então $P$ tem um subconjunto $\tau$-controlado $Q$ tal que

$$
\{a \mid(a, r) \in P \text { para algum } r\} \subset \bigcup\{\mathbb{B}(a, r) \mid(a, r) \in Q\} .
$$

Demonstração. Seja $\Omega \subset 2^{P}$ a classe de todos os subconjuntos $\tau$-controlados $Q$ de $P$ com a seguinte propriedade: para todo $(b, s) \in P$,

$$
\begin{aligned}
& \text { ou } d(a, b) \leq r \text { para algum }(a, r) \in Q, \\
& \text { ou } d(a, b)>r>s / \tau \text { para todo }(a, r) \in Q .
\end{aligned}
$$

Note que $\Omega$ é não vazio (pois $\emptyset \in \Omega$ ) e está parcialmente ordenado pela inclusão. Dado um subconjunto $T$ totalmente ordenado em $\Omega, Q:=\bigcup T \in \Omega$ é uma cota superior para $T$. Podemos, pois, aplicar o Lema de Zorn para concluir que $\Omega$ tem um elemento maximal. Seja $Q$ um maximal de $\Omega$. O lema se cumpre, pois, caso contrário, temos

$$
K:=P \cap\{(b, s) \mid d(a, b)>r, \text { para todo }(a, r) \in Q\} \neq \emptyset .
$$

Podemos escolher $(c, t) \in K \operatorname{com} \tau t>\sup \{s \mid(b, s) \in K\}$ (pois $\tau>1$ e o sup no segundo membro é finito). Definamos $\widetilde{Q}:=Q \cup\{(c, t)\}$. Então $(c, t) \notin Q$ (caso contrário $(c, t) \in Q \cap K$, o que acarreta a contradição $0=d(c, c)>t), \operatorname{logo} Q \subsetneq \widetilde{Q}$.

Para todo $(a, r) \in Q$, temos $d(a, c)>r$. Daí, como $(c, t) \in P$ e $Q \in \Omega$, para $(b, s)=(c, t)$ deve valer a segunda condição na definição de $\Omega$, i.e. $d(a, c)>r>t / \tau$ para todo $(a, b) \in Q$. Então $\widetilde{Q}$ é $\tau$-controlada. 
Agora, suponhamos que existe $(b, s) \in P$ tal que a primeira condição na definição de $\Omega$ não valha para $\widetilde{Q}$, i.e. $d(a, b)>r$ para todo $(a, r) \in \widetilde{Q}$. Em particular, como $Q \subset \widetilde{Q}$, temos que a última desigualdade vale para todo $(a, r) \in Q$, e tendo em vista que $Q \in \Omega$ isso implica $d(a, b)>r>s / \tau$ para todo $(a, r) \in Q$. Daí $(b, s) \in K, \operatorname{logo} t>s / \tau$ pela escolha de $(c, t)$, e $d(c, b)>t$ vale pela hipótese assumida sobre $(b, s)$ com $(a, r)=(c, t)$. Assim, $(b, s)$ cumpre a segunda condição da definição de $\Omega$ para $\widetilde{Q}$.

Portanto $\widetilde{Q} \in \Omega$ o que contradiz a maximalidade de $Q$. Está provado que $K=\emptyset$, o que significa que, para todo $(b, s) \in P$, existe $(a, r) \in Q$ tal que $d(a, b) \leq r$, i.e. $b \in \mathbb{B}(a, r)$.

Prova do Teorema 2.22. Escolhendo $\tau$ de modo a satisfazer (2.1) do Teorema 2.18 e aplicando o Lema $2.23 \mathrm{com}$

$$
P:=\{(a, r) \mid a \in A, \mathbb{B}(a, r) \in \mathcal{F}\} \subset X \times\{r \mid 0<r<\lambda\},
$$

obtemos um subconjunto $\tau$-controlado $Q$ de $P$ tal que $A$ é coberto pela família de bolas fechadas

$$
\mathcal{G}:=\{\mathbb{B}(a, r) \mid(a, r) \in Q\} \subset \mathcal{F} .
$$

Pelo Corolário 2.19 , temos que $\mathcal{G}$ é a união de $2 \zeta+1$ subfamílias disjuntas $\mathcal{G}_{1}, \ldots, \mathcal{G}_{2 \zeta+1} \subset \mathcal{G} \subset \mathcal{F}$. Portanto,

$$
A \subset \bigcup_{j=1}^{2 \zeta+1} \bigcup \mathcal{G}_{j} .
$$

Corolário 2.24. Sejam $(X, d)$ um espaço métrico, $A \subset X$ separável, $d(\xi, \eta, \zeta)$-direcionalmente limitada em $A, 0<\lambda<\xi / 2, \mathcal{F}$ uma família de bolas fechadas em $X$ com raios estritamente positivos e menores que $\lambda$. Suponha que cada ponto de $A$ é o centro de algum membro de $\mathcal{F} e$ $\inf \{r \mid \mathbb{B}(a, r) \in \mathcal{F}\}=0$ para todo $a \in A$. Então $\mathcal{F}$ é $\mu$-adequada para $A$.

Demonstração. Mostraremos que a hipótese do Teorema 2.2 se satisfaz com

$$
\mathcal{K}=\{A\} \quad \text { e } \quad \sigma(A)=\frac{2 \zeta}{2 \zeta+1}<1 .
$$

Seja $W$ aberto em $X$. Podemos supor que $\mu(A \cap W)<\infty$, caso contrário a desigualdade na hipótese do teorema 2.2 se verifica trivialmente. Cada ponto de $A \cap W$ é o centro de algum membro da família

$$
\mathcal{F}^{\prime}:=\mathcal{F} \cap\{\mathbb{B}(a, r) \mid a \in A, r>0 \text { e } \mathbb{B}(a, r) \subset W\} .
$$

Portanto, o Teorema 2.22 implica que $A \cap W$ está contido na união de $2 \zeta+1$ subfamílias disjuntas $\mathcal{H}_{1}, \mathcal{H}_{2}, \ldots, \mathcal{H}_{2 \zeta+1}$ de $\mathcal{F}^{\prime}$ i.e.

$$
A \cap W \subset \bigcup_{j=1}^{2 \zeta+1} \bigcup \mathcal{H}_{j} .
$$

Como $A$ é separável, qualquer família de conjuntos com interior não vazio em $A$ é enumerável. Assim, cada $\mathcal{H}_{j}$ é enumerável e, portanto, $\bigcup \mathcal{H}_{j}$ é um F- $\sigma$ e, em particular, um boreliano. Por outro lado, a desigualdade

$$
\sum_{j=1}^{2 \zeta+1} \mu\left(A \cap \bigcup \mathcal{H}_{j}\right) \geq \mu(A \cap W),
$$

permite escolher um inteiro $j$ para o qual

$$
\mu\left(A \cap \bigcup \mathcal{H}_{j}\right) \geq \frac{\mu(A \cap W)}{2 \zeta+1} .
$$


Daí,

$$
\begin{aligned}
\mu\left((A \cap W) \backslash \bigcup \mathcal{H}_{j}\right) & =\mu\left(A \cap\left(W \backslash \bigcup \mathcal{H}_{j}\right)\right)= \\
& =\mu\left\llcorner A\left(W \backslash \bigcup \mathcal{H}_{j}\right)^{\mu} \stackrel{A}{A \text { boreliana }}=\right. \\
& =\mu\left\llcorner A(W)-\mu\left\llcorner A\left(\bigcup \mathcal{H}_{j}\right)=\mu(A \cap W)-\mu\left(A \cap \bigcup \mathcal{H}_{j}\right) \leq\right.\right. \\
& \leq \mu(A \cap W)-\frac{\mu(A \cap W)}{2 \zeta+1}=\left(1-\frac{1}{2 \zeta+1}\right) \mu(A \cap W) .
\end{aligned}
$$

Portanto,

$$
\mu\left((A \cap W) \backslash \bigcup \mathcal{H}_{j}\right) \leq \frac{2 \zeta}{2 \zeta+1} \mu(A \cap W)=\sigma(A) \mu(A \cap W),
$$

mostrando que $\mathcal{F}$ é $\mu$-adequada para $\bigcup \mathcal{K}=A$, pelo Teorema 2.2 .

Para o Corolário 2.26 vamos usar a Propriedade de Vitali simétrica (SVP), descrita na Definição 3.10 em [Sim14, pág. 17].

Definição 2.25 (Propriedade de Vitali simétrica (SVP)). Uma medida $\mu$ num espaço métrico $X$ satisfaz a propriedade de Vitali simétrica se, para todo $A \subset X \operatorname{com} \mu(A)<\infty$ e para toda cobertura $\mathcal{F}$ de $A$ for bolas fechadas com raios estritamente positivos que cubra $A$ finamente no sentido forte (i.e. para todo $x \in A$, temos $\inf \{r>0 \mid \mathbb{B}(x, r) \in \mathcal{F}\}=0$ ), existe uma subfamília enumerável disjunta $\mathcal{G} \subset \mathcal{F}$ tal que $\mu(A \backslash \bigcup \mathcal{G})=0$.

Corolário 2.26. Sejam $(X, d)$ um espaço métrico separável $(\xi, \eta, \zeta)$-direcionalmente limitado, e $\mu$ uma medida exterior Boreliana finita nos limitados de $X$. Então $\mu$ satisfaz a propriedade de Vitali simétrica.

Demonstração. Sejam $A \subset X \operatorname{com} \mu(A)<\infty$ e $\mathcal{F}$ uma família de bolas fechadas com raios estritamente positivos que cobre $A$ finamente no sentido forte. Em particular, $A$ é separável e, substituindose $\mathcal{F}$ por $\mathcal{F}^{\prime}:=\{B \in \mathcal{F} \mid B=\mathbb{B}(a, r), r<\xi / 4\}$, se necessário, a hipótese do Corolário 2.24 se verifica. Daí, pelo referido corolário, temos que $\mathcal{F}$ é $\mu$-adequada para $A$, i.e para todo $U \subset X$ aberto existe $\mathcal{G} \subset \mathcal{F}$ disjunta enumerável tal que $\cup \mathcal{G} \subset U$ e $\mu((U \cap A) \backslash \cup \mathcal{G})=0$. Em particular para $U=X$, obtém-se $\mathcal{G} \subset \mathcal{F}$ disjunta enumerável tal que $\mu(A \backslash \bigcup \mathcal{G})=0$.

\subsection{Relações de cobertura}

Apresentaremos agora a noção de $\mu$-relação de Vitali, a qual será usada na teoria de derivação a ser discutida no Capítulo 3. Lembre que $\mu$ é uma medida de Borel em $X$, finita nas partes limitadas de $X$, fixada no início deste capítulo.

Definição 2.27 (Relação de cobertura). Uma relação de cobertura em $X$ é um subconjunto $C$ de

$$
\{(x, S) \mid x \in S \subset X\} .
$$

Para a relação de cobertura $C$ em $X$ e $Z \subset X$, denotamos por $C(Z)$ o subconjunto de $2^{X}$ dado por

$$
C(Z):=\{S \subset X \mid \exists x \in Z,(x, S) \in C\} .
$$

Definição 2.28 (Relação de cobertura fina). Dados $x \in X$ e $C$ uma relação de cobertura em $X$, diz-se que $C$ é fina em $x$ se,

$$
\inf \{\operatorname{diam} S \mid(x, S) \in C\}=0 .
$$


Definição 2.29 (Relação de Vitali). Seja $V$ uma relação de cobertura em $X$. Diz-se que $V$ é uma $\mu$-relação de Vitali em $X$ se:

i) $V(X) \subset \mathscr{B}_{X}$.

ii) Para todo $x \in X, V$ é fina em $x$.

iii) Para todo $Z \subset X$ e todo $C \subset V$ tal que $C$ é fina em todo ponto de $Z$, existe uma subfamília enumerável disjunta $\mathcal{F}$ de $C(Z)$ tal que $\mu(Z \backslash \bigcup \mathcal{F})=0$.

Observação 2.30. Note que, se $V=\{(x, \mathbb{B}(x, r)) \mid x \in X, r>0\}$, e se $\mu$ for finita, então $V$ é uma $\mu$-relação de Vitali em $X$ se, e somente se, $\mu$ tem a propriedade de Vitali simétrica (SVP) descrita na Definição 2.25. Para uma medida $\mu$ não necessariamente finita, vale a implicação: se $V$ for uma $\mu$-relação de Vitali em $X$, então $\mu$ tem SVP.

Definição 2.31 (Limite ao longo de uma cobertura). Sejam $C$ uma relação de cobertura em $X$ tal que $C$ é fina em $x \in X$ e $f$ uma função de conjunto a valores em $\overline{\mathbb{R}}$. Definimos

$(C) \limsup _{x} f=(C) \limsup _{S \rightarrow x} f(S):=\lim _{\epsilon \rightarrow 0^{+}} \sup \{f(S) \mid(x, S) \in C, \operatorname{diam} S \leq \epsilon, S \in \operatorname{dom} f\}$,

$(C) \liminf _{x} f=(C) \liminf _{S \rightarrow x} f(S):=\lim _{\epsilon \rightarrow 0^{+}} \inf \{f(S) \mid(x, S) \in C, \operatorname{diam} S \leq \epsilon, S \in \operatorname{dom} f\}$.

Usaremos $(C) \lim _{x}$ nos pontos onde $(C) \limsup _{x}$ coincide com $(C) \liminf _{x}$ i.e.

$$
(C) \lim _{x} f:=(C) \limsup _{x} f=(C) \liminf _{x} f .
$$

Observação 2.32. Na definição acima, podemos substituir $\lim _{\epsilon \rightarrow 0^{+}}$por $\inf _{\epsilon>0}$ no caso do $(C) \lim \sup$ ou por $\sup _{\epsilon>0}$ no caso do $(C) \lim$ inf i.e.

$$
\begin{aligned}
& (C) \limsup _{x} f=\inf _{\epsilon>0} \sup \{f(S) \mid(x, S) \in C, \operatorname{diam} S \leq \epsilon, S \in \operatorname{dom} f\} \\
& (C) \liminf _{x} f=\sup _{\epsilon>0} \inf \{f(S) \mid(x, S) \in C, \operatorname{diam} S \leq \epsilon, S \in \operatorname{dom} f\} .
\end{aligned}
$$

Definição 2.33. Dados $1<\tau<\infty$ e $\delta: V(X) \rightarrow[0, \infty)$, definimos, para cada $S \in V(X)$, sua $(\delta, \tau)$-ampliação como na Definição 2.5 , i.e.

$$
\widehat{S}=\bigcup\{T \mid T \in V(X), T \cap S \neq \emptyset, \delta(T) \leq \tau \delta(S)\}
$$

Note que $\widehat{S}$ depende de $V(X)$; se for necessário indicar esta dependência, usaremos a notação $\widehat{S}_{V(X)}$.

A seguir, investigaremos alguns critérios para que uma relação de cobertura seja uma $\mu$-relação de Vitali. Iniciemos com o enunciado de um teorema de Federer:

Teorema ([Fed96, 2.8.17]). Sejam $V$ uma relação de cobertura em $X$ com $V(X)$ contida nas partes fechadas limitadas de $X$ e $V$ fina em cada ponto de $X, \delta: V(X) \rightarrow[0, \infty)$ e $1<\tau<\infty$. Para cada $S \in V(X)$, denotamos por $\widehat{S}$ a $(\delta, \tau)$-ampliação de $S$. Suponha que, para $\mu$-q.t. $x \in X$,

$$
0 \leq(V) \limsup _{S \rightarrow x}\left(\delta(S)+\frac{\mu(\widehat{S})}{\mu(S)}\right)<\infty .
$$

Então $V$ é uma $\mu$-relação de Vitali em $X$.

Parece-nos que o enunciado acima contém uma pequena imprecisão, conforme mostra o contraexemplo abaixo. A demonstração dada por Federer tem um problema na chamada que se faz ao Teorema 2.8.7 (que corresponde ao nosso Teorema 2.8); no referido teorema, a hipótese exige uma 
desigualdade estrita $\mu(\widehat{S})<\lambda \mu(S)$, mas o argumento do Federer, pelo que apuramos, só permite concluir a desigualdade não estrita. Esta pequena imprecisão será corrigida no Teorema 2.34, abaixo, colocando-se a hipótese adicional de que, para $\mu$-q.t. $x \in X, \mu$ é estritamente positiva em todo $S \in V(\{x\})$.

Contra-exemplo para o enunciado de 2.8.1\%. Seja $X=\mathbb{R}^{n}, \mu=\mathcal{L}^{n}$ e

$$
V=\left\{(x, \mathbb{B}(x, r)) \mid x \in \mathbb{R}^{n}, 0 \leq r<\infty\right\}
$$

(atenção: bolas degeneradas fazem parte de $V$ ). Tome $\delta:=\operatorname{diam}$ e $\tau=2$. Então $V(X) \subset$ $\{$ fechados e limitados de $X\}$ e, para todo $x \in X, V$ é fina em $x$ e

$$
0 \leq(V) \limsup _{S \rightarrow x}\left(\delta(S)+\frac{\mu(\widehat{S})}{\mu(S)}\right)=5^{n}<\infty .
$$

Por outro lado, tomando $Z=\mathbb{R}^{n}$ (ou qualquer conjunto de medida estritamente positiva) e $C=$ $\left.\{(x,\{x\}\}) \mid x \in \mathbb{R}^{n}\right\} \subset V$, tem-se $C(Z)=\{$ singletons de $Z\}$, o qual não admite subconjunto enumerável que cubra $\mu$-q.t. $Z$. Portanto, $V$ não é uma $\mu$-relação de Vitali em $X$.

Teorema 2.34. Sejam $V$ uma relação de cobertura em $X$ com $V(X)$ contida nas partes fechadas limitadas de $X$ e $V$ fina em cada ponto de $X, \delta: V(X) \rightarrow[0, \infty)$ e $1<\tau<\infty$. Para cada $S \in V(X)$, denotamos por $\widehat{S}$ a $(\delta, \tau)$-ampliação de $S$, como na Definição 2.33. Suponhamos que, para $\mu$-q.t. $x \in X, \mu$ é estritamente positiva em todo $S \in V(\{x\})$ e

$$
0 \leq(V) \limsup _{S \rightarrow x}\left(\delta(S)+\frac{\mu(\widehat{S})}{\mu(S)}\right)<\infty .
$$

Então $V$ é uma $\mu$-relação de Vitali em $X$.

Demonstração. Da hipótese segue diretamente que $V(X) \subset \mathscr{B}_{X}$ e que $V$ é fina em cada ponto de $X$. Resta provar a condição iii) na definição de $\mu$-relação de Vitali. Suponha $Z \subset X$ e $C \subset V$ fina em cada ponto de $Z$. Sem perda de generalidade, podemos supor que $Z \subset\{x \mid \forall S \in V(\{x\}), \mu(S)>0\}$, pois o complementar do último conjunto é $\mu$-nulo por hipótese.

Defina, para cada $n \in \mathbb{N}$,

$$
\begin{aligned}
& C_{n}:=C \cap\{(x, S) \mid x \in Z, \delta(S)+\mu(\widehat{S}) / \mu(S)<n\}, \\
& A_{n}:=Z \cap\left\{x \mid 0 \leq(V) \limsup _{S \rightarrow x}\left(\delta(S)+\frac{\mu(\widehat{S})}{\mu(S)}\right)<n\right\} .
\end{aligned}
$$

Afirmo que $C_{n}$ é uma relação de cobertura em $X$ fina em cada ponto de $A_{n}$. Com efeito, é claro que é uma relação de cobertura, pois é um subconjunto de $V$. Além disso, dado $x \in A_{n}$, o fato de que $0 \leq(V) \lim \sup _{S \rightarrow x}\left(\delta(S)+\frac{\mu(\widehat{S})}{\mu(S)}\right)<n$ implica que existe $\epsilon_{0}>0$ tal que, para $0<\epsilon<\epsilon_{0}$, tem-se

$$
0 \leq \sup \left\{\delta(S)+\frac{\mu(\widehat{S})}{\mu(S)} \mid(x, S) \in V, \operatorname{diam} S \leq \epsilon\right\}<n .
$$

O mesmo vale com $C$ no lugar de $V$ na linha acima, pois $C$ é fina em $x$ (logo vale o $0 \leq$ sup, i.e. o conjunto onde se está tomando o supremo é não vazio) e $C \subset V(\operatorname{logo}$ vale o sup $<n)$. Portanto, para $0<\epsilon<\epsilon_{0}$, existe $S \in C\{x\}$ tal que diam $S \leq \epsilon$ e $\delta(S)+\frac{\mu(\widehat{S})}{\mu(S)}<n$, i.e. para $0<\epsilon<\epsilon_{0}$ existe $S \in C_{n}(\{x\})$ com diam $S \leq \epsilon$, o que mostra que $C_{n}$ é fina em $x$, como afirmado. 
Assim, $C_{n}\left(A_{n}\right)$ é uma coleção de fechados de $X$ que cobre $A_{n}$ finamente, $\left.\delta\right|_{C_{n}\left(A_{n}\right)}: C_{n}\left(A_{n}\right) \rightarrow$ $[0, \infty)$ é limitada por $n$ e, para todo $S \in C_{n}\left(A_{n}\right)$, tem-se $\frac{\mu(\widehat{S})}{\mu(S)}<n$ (lembre que $A_{n} \subset Z \subset$ $\{x \mid \forall S \in V(\{x\}), \mu(S)>0\})$. Aqui, $\widehat{S}$ denota a $(\delta, \tau)$-ampliação de $S$ com respeito a $V(X)$ e $\delta: V(X) \rightarrow[0, \infty)$; denotando por $\widehat{S}_{C_{n}\left(A_{n}\right)}$ a $(\delta, \tau)$-ampliação de $S$ com respeito a $C_{n}\left(A_{n}\right)$ e $\left.\delta\right|_{C_{n}\left(A_{n}\right)}: C_{n}\left(A_{n}\right) \rightarrow[0, \infty)$, é claro que $\widehat{S}_{C_{n}\left(A_{n}\right)} \subset \widehat{S}$, portanto $\mu\left(\widehat{S}_{C_{n}\left(A_{n}\right)}\right)<n \mu(S)$.

Podemos aplicar, pois, o Teorema $2.8 \mathrm{com} A_{n}$ no lugar de $A, C_{n}\left(A_{n}\right)$ no lugar de $\mathcal{F},\left.\delta\right|_{C_{n}\left(A_{n}\right)}$ e $\lambda=n+1>1$. Conclui-se, pois, que $C_{n}\left(A_{n}\right)$ é $\mu$-adequada para $A_{n}$. Como $C_{n}\left(A_{n}\right) \subset C(Z)$, segue-se que $C(Z)$ é $\mu$-adequada para $A_{n}$, para todo $n \in \mathbb{N}$. Do Corolário 2.3, conclui-se que $C(Z)$ é $\mu$-adequada para $\bigcup_{n \in \mathbb{N}} A_{n}$, portanto $\mu$-adequada para $Z$, uma vez que $\mu\left(Z \backslash \bigcup_{n \in \mathbb{N}} A_{n}\right)=0$.

Teorema 2.35. Seja $\mathcal{K} \subset 2^{X}$ enumerável tal que $X=\bigcup \mathcal{K}$. Suponha que a métrica $d$ de $X$ é $(\xi, \eta, \zeta)$-direcionalmente limitada cada membro de $\mathcal{K}$, e que $X$ seja separável. Então

$$
V:=\{(x, \mathbb{B}(x, r)) \mid x \in X, 0<r<\infty\}
$$

é uma $\mu$-relação de Vitali em $X$.

Demonstração. É claro que $V(X) \subset \mathscr{B}_{X}$ e que $V$ é fina em cada ponto de $X$. Sejam $Z \subset X$ e $C \subset V$ tais que $C$ é fina em cada ponto de $Z$. Para cada $A \in \mathcal{K}$, aplicando o Corolário 2.24 com $A \cap Z$ no lugar de $A$, temos que $C(Z)$ é $\mu$-adequada para $A \cap Z$. Daí, como $\mathcal{K}$ é enumerável, segue do Corolário 2.3 que $C(Z)$ é $\mu$-adequada para $Z \cap(\bigcup \mathcal{K})=Z$.

Corolário 2.36. Com a mesma hipótese do Teorema 2.35, $\mu$ tem a propriedade de Vitali simétrica em $X$.

Demonstração. Ver o Corolário 2.26 e a Observação 2.30.

Exemplo 2.37. O Teorema 2.35 e o Corolário 2.36 valem, em particular, se $X$ for uma variedade Riemanniana munida da métrica Riemanniana $d$. De fato, $X$ é $\sigma$-compacta (i.e. é a união de uma coleção enumerável de conjuntos compactos) e, pelo Exemplo 2.14, $d$ é direcionalmente limitada em todo compacto de $X$.

Para o seguinte teorema, a medida $\mu$ sobre $X$ pode ser qualquer, i.e. podemos dispensar as restrições sobre $\mu$ impostas no início do capítulo.

Teorema 2.38. Seja $\left(\mathcal{P}_{i}\right)_{i \in \mathbb{N}}$ uma sequência de partições borelianas enumeráveis de $X$ tal que:

i) cada membro de $\mathcal{P}_{1}$ é limitado;

ii) para todo $i \in \mathbb{N}$, cada membro de $\mathcal{P}_{i}$ é reunião de uma subfamília de $\mathcal{P}_{i+1}$;

iii) $\sup \left\{\operatorname{diam} S \mid S \in \mathcal{P}_{i}\right\} \stackrel{i \rightarrow \infty}{\rightarrow} 0$

Então $V=\left\{(x, S) \mid \exists j \in \mathbb{N}, S \in \mathcal{P}_{j}, x \in S\right\}$ é uma $\mu$-relação de Vitali.

Demonstração. Pelas hipóteses, para cada $i \in \mathbb{N}, \mathcal{P}_{i}$ é uma partição Boreliana de $X$ então $\mathcal{P}_{i}$ é uma família enumerável disjunta de Borelianos com $\bigcup \mathcal{P}_{i}=X$. É claro que $V(X)=\bigcup_{i \in \mathbb{N}} \mathcal{P}_{i} \subset \mathscr{B}_{X}$, o qual é enumerável pois cada $\mathcal{P}_{i}$ é enumerável. Em vista da condição iiii) $V$ é fina em cada ponto de $X$.

Sejam $Z \subset X$ e $C \subset V$ tal que $C$ seja fina em cada ponto de $Z$. Para cada $x \in Z$, definamos $N_{x}:=\left\{i \in \mathbb{N} \mid \exists S \in C(Z) \cap \mathcal{P}_{i}, x \in S\right\}$. Então $N_{x}$ é não vazio, logo possui um menor elemento; sejam $i_{x}:=\min N_{x}$ e $S_{x}$ o único membro de $C(Z) \cap \mathcal{P}_{i_{x}}$ tal que $x \in S_{x}$. Note que esta unicidade decorre do fato de que cada $\mathcal{P}_{i}$ é disjunta. 
Segue da condição $i i$ ) que, para todo $S \in C(Z)$ tal que $x \in S$, tem-se $S \subset S_{i_{x}}$. Daí, se $x \neq y \in Z$, ou $S_{x}=S_{y}$, ou $S_{x} \cap S_{y}=\emptyset$; pois, se $S_{x} \cap S_{y} \neq \emptyset$, então $i_{x}=i_{y}$ porque se $i_{x}<i_{y}$, teríamos $S_{y} \subset S_{x}$, contrariando a minimalidade de $i_{y}$, e de forma análoga não se pode ter $i_{y}<i_{x}$. Assim, $S_{x}=S_{y}$ pelo fato de $\mathcal{P}_{i_{x}}$ ser disjunta. Então $\left\{S_{i_{x}} \mid x \in Z\right\}$ é uma subfamília disjunta de $C(Z)$ que cobre $Z$. Tal subfamília é enumerável, pois $V(X)=\bigcup_{i \in \mathbb{N}} \mathcal{P}_{i}$ é enumerável, uma vez que cada $\mathcal{P}_{i}$ é enumerável. 


\section{Capítulo 3}

\section{Diferenciação de medidas}

Para uma classe de medidas Borel-regular com propriedades de finitude adequada em espaços métricos, definiremos a noção de derivada de uma medida em relação a outra. Serão estudadas propriedades desta noção de diferenciabilidade e versões abstratas do Teorema Fundamental do Cálculo nesse contexto. A seguir, serão introduzidas noções de densidades de medidas, com as quais poderemos reformular as noções clássicas de limite e continuidade de funções no âmbito da teoria da medida, resultando nas noções chamadas de "limite e continuidade aproximada". Finalmente, na Secção 3.4, combinaremos estes fatos sobre a teoria de diferenciação de medidas para apresentar a fórmula da área para aplicações contínuas entre espaços métricos, sob suposições de regularidade mínima no Teorema 3.29.

Notação. Neste capítulo, fixamos um espaço métrico $(X, d)$ e denotamos por $\mathcal{M}$ o conjunto de todas as medidas (exteriores) Borel-regulares em $X$, finitas nas partes limitadas de $X$. Fixamos $\mu \in \mathcal{M}$ e $V$ uma $\mu$-relação de Vitali, como na Definição 2.29.

Definição 3.1. Para cada $\nu \in \mathcal{M}$, associaremos a medida $\nu_{\mu}$ definida, para todo $A \subset X$, por:

$$
\nu_{\mu}(A):=\inf \left\{\nu(B) \mid B \in \mathscr{B}_{X}, \mu(A \backslash B)=0\right\} .
$$

No Teorema 3.3 será mostrado que $\nu_{\mu}$ é, de fato, uma medida Borel-regular em $X$. Também será provado que $\nu_{\mu}$ coincide com a parte absolutamente contínua da decomposição de Lebesgue de $\nu$ com respeito a $\mu$. Preliminarmente, note que, pelo fato de ser $\nu$ Borel-regular, $\nu_{\mu} \leq \nu$.

\subsection{Derivadas}

Os resultados centrais desta seção são o Teorema 3.8, onde mostraremos que a derivada $\mathrm{D}(\nu, \mu, V, x)$ (Definição 3.5) existe $\mu$-q.s., e o Teorema 3.10, onde mostramos que a medida $\nu_{\mu}$ pode ser obtida integrando $\mathrm{D}(\nu, \mu, V, x)$, o que corresponde ao Teorema Fundamental do Cálculo nesse contexto.

Definição 3.2. Com $\nu \in \mathcal{M}$ e $\mu \in \mathcal{M}$, dizemos que:

i) $\nu$ é absolutamente contínua em relação a $\mu$ se para todo $A \subset X, \mu(A)=0$ implica $\nu(A)=0$. Notação: $\nu \ll \mu$.

ii) $\nu$ e $\mu$ são mutuamente singulares se existe um $B \in \mathscr{B}_{X}$ tal que $\nu(B)=\mu(X \backslash B)=0$. Notação: $\nu \perp \mu$.

Teorema 3.3. Para cada $\nu \in \mathcal{M}$, existe um $B \in \mathscr{B}_{X}$ tal que

$$
\nu_{\mu}=\nu\llcorner B \quad \text { e } \quad \mu(X \backslash B)=0 .
$$

Portanto, $\nu_{\mu} \in \mathcal{M}, \nu\left\llcorner B^{c} \in \mathcal{M}\right.$ e $\nu=\nu_{\mu}+\nu\left\llcorner B^{c}\right.$. Além disso, $\nu_{\mu} \ll \mu e \nu\left\llcorner B^{c} \perp \mu\right.$; a igualdade $\nu=\nu_{\mu}$ vale se, e somente se, $\nu$ é absolutamente contínua com relação a $\mu$. 


\section{Demonstração.}

1) Mostremos que $\nu_{\mu}$ é uma medida de Borel em $X$.

a) Inicialmente, verifiquemos que $\nu_{\mu}$ é uma medida em $X$. É claro que $\nu_{\mu}(\emptyset)=0$. Para provar a subaditividade enumerável, seja $\left(A_{i}\right)_{i \in \mathbb{N}}$ uma sequência de subconjuntos de $X$ tal que $\sum_{n \in \mathbb{N}} \nu_{\mu}\left(A_{n}\right)<\infty$. Fixe $\epsilon>0$. Pela definição de $\nu_{\mu}$, para cada $i \in \mathbb{N}$, existe $E_{i} \in \mathscr{B}_{X}$ tal que $\mu\left(A_{i} \backslash E_{i}\right)=0$ e

$$
\nu\left(E_{i}\right) \leq \nu_{\mu}\left(A_{i}\right)+\epsilon 2^{-i} .
$$

Portanto, $\cup_{i \in \mathbb{N}} E_{i} \in \mathscr{B}_{X}$ e

$$
\begin{aligned}
\mu\left(\left(\bigcup_{i \in \mathbb{N}} A_{i}\right) \backslash \bigcup_{j \in \mathbb{N}} E_{j}\right) & =\mu\left(\left(\bigcup_{i \in \mathbb{N}} A_{i}\right) \bigcap\left(\bigcap_{j \in \mathbb{N}} E_{j}^{c}\right)\right)=\mu\left(\bigcup_{i \in \mathbb{N}}\left(A_{i} \bigcap\left(\bigcap_{j \in \mathbb{N}} E_{j}^{c}\right)\right)\right) \leq \\
& \leq \sum_{i \in \mathbb{N}} \mu\left(A_{i} \bigcap\left(\bigcap_{j \in \mathbb{N}} E_{j}^{c}\right)\right) \leq \\
& \leq \sum_{i \in \mathbb{N}} \mu\left(A_{i} \backslash E_{i}\right)=0 .
\end{aligned}
$$

Logo,

$$
\nu_{\mu}\left(\bigcup_{i \in \mathbb{N}} A_{i}\right) \leq \nu\left(\bigcup_{i \in \mathbb{N}} E_{i}\right) \leq \sum_{i \in \mathbb{N}} \nu\left(E_{i}\right) \leq \sum_{i \in \mathbb{N}} \nu_{\mu}\left(A_{i}\right)+\epsilon .
$$

Pela arbitrariedade de $\epsilon>0$ fixado, segue

$$
\nu_{\mu}\left(\bigcup_{i \in \mathbb{N}} A_{i}\right) \leq \sum_{i \in \mathbb{N}} \nu_{\mu}\left(A_{i}\right),
$$

como queríamos.

b) Verifiquemos, pelo critério de Carathéodory, Teorema 1.20, que todos os borelianos são $\nu_{\mu^{-}}$ mensuráveis. Sejam $A_{1}, A_{2} \subset X$ tais que $\delta:=d\left(A_{1}, A_{2}\right)>0$. Pondo $A:=A_{1} \cup A_{2}$, mostremos que $\nu_{\mu}\left(A_{1}\right)+\nu_{\mu}\left(A_{2}\right) \leq \nu_{\mu}(A)$. Podemos supor que $\nu_{\mu}\left(A_{1}\right)<\infty$ e $\nu_{\mu}\left(A_{2}\right)<\infty$, caso contrário $\nu_{\mu}(A)=\infty$ por monotonicidade e a desigualdade se verifica trivialmente. Dado $\epsilon>0$, tomemos $E \in \mathscr{B}_{X}$ tal que

$$
\mu(A \backslash E)=0 \quad \text { e } \quad \nu(E) \leq \nu_{\mu}(A)+\frac{\epsilon}{2} .
$$

Cortando $E$ com a união das vizinhanças abertas de raio $\delta / 2$ de $A_{1}$ e $A_{2}$, podemos supor $E:=E_{1} \cup E_{2} \operatorname{com} E_{i}$ boreliano contido na vizinhança aberta de raio $\delta / 2$ de $A_{i}$ para $i \in\{1,2\}$.

Como $A \backslash E=\left(A_{1} \backslash E_{1}\right) \cup\left(A_{2} \backslash E_{2}\right)$ e $d\left(A_{1} \backslash E_{1}, A_{2} \backslash E_{2}\right) \geq d\left(A_{1}, A_{2}\right)>0$, o Critério de Carathéodory aplicado à medida boreliana $\mu$ fornece $\mu\left(A_{1} \backslash E_{1}\right)+\mu\left(A_{2} \backslash E_{2}\right)=\mu(A \backslash E)=0$, $\operatorname{logo} \mu\left(A_{i} \backslash E_{i}\right)=0$ para $i \in\{1,2\}$. Portanto,

$$
\nu_{\mu}\left(A_{1}\right)+\nu_{\mu}\left(A_{2}\right) \leq \nu\left(E_{1}\right)+\nu\left(E_{2}\right)=\nu(E) \leq \nu_{\mu}(A)+\epsilon .
$$

Pela arbitrariedade de $\epsilon>0$ tomado, segue a desigualdade que queríamos mostrar.

2) Seja $A \subset X$ um boreliano. Afirmamos que existe $B \subset A$ boreliano tal que $\nu_{\mu}(A)=\nu(B)$ e $\mu(A \backslash B)=0$. Com efeito, para cada $n \in \mathbb{N}$, escolhamos $D_{n} \in \mathscr{B}_{X}$ tal que

$$
\mu\left(A \backslash D_{n}\right)=0 \quad \text { e } \quad \nu\left(D_{n}\right) \leq \nu_{\mu}(A)+\frac{1}{n} .
$$


Substituindo $D_{n}$ por $D_{n} \cap A \in \mathscr{B}_{X}$, se necessário, podemos supor que $D_{n} \subset A$.

Pondo $B:=\bigcap_{n \in \mathbb{N}} D_{n} \subset A$, temos $B \in \mathscr{B}_{X}$ e

$$
\begin{aligned}
\mu(A \backslash B) & =\mu\left(A \backslash\left(\bigcap_{n \in \mathbb{N}} D_{n}\right)\right)=\mu\left(A \cap\left(\bigcup_{n \in \mathbb{N}} D_{n}^{c}\right)\right)=\mu\left(\bigcup_{n \in \mathbb{N}}\left(A \cap D_{n}^{c}\right)\right) \leq \\
& \leq \sum_{n \in \mathbb{N}} \mu\left(A \cap D_{n}^{c}\right)=\sum_{n \in \mathbb{N}} \mu\left(A \backslash D_{n}\right)=0,
\end{aligned}
$$

portanto $\nu_{\mu}(A) \leq \nu(B)$. Além disso para todo $i \in \mathbb{N}$,

$$
\nu(B)=\nu\left(\bigcap_{n \in \mathbb{N}} D_{n}\right) \leq \nu\left(D_{i}\right) \leq \nu_{\mu}(A)+\frac{1}{i} .
$$

Como $i$ é arbitrário, segue $\nu(B) \leq \nu_{\mu}(A)$, portanto $\nu_{\mu}(A)=\nu(B)$, como queríamos.

3) Sejam $A \subset X$ um boreliano limitado e $B \in \mathscr{B}_{X}$ dado pelo item anterior, i.e. tal que $B \subset A$, $\nu_{\mu}(A)=\nu(B)$ e $\mu(A \backslash B)=0$. Mostremos que, para todo $S \subset A, \nu_{\mu}(S)=\nu(B \cap S)$. Com efeito:

a) Caso 1: $S$ é um boreliano contido em A. Como $B \cap S \in \mathscr{B}_{X}$ e $S \backslash(B \cap S)=S \backslash B \subset A \backslash B$ temos $\mu(S \backslash(B \cap S)) \leq \mu(A \backslash B)=0$, portanto

$$
\nu_{\mu}(S) \leq \nu(B \cap S) .
$$

Analogamente, substituindo-se $S$ por $A \backslash S$, conclui-se que

$$
\nu_{\mu}(A \backslash S) \leq \nu(B \backslash S) .
$$

Logo,

$$
\nu_{\mu}(S)+\nu_{\mu}(A \backslash S)=\nu_{\mu}(A)=\nu(B)=\nu(B \cap S)+\nu(B \backslash S) \geq \nu_{\mu}(S)+\nu_{\mu}(A \backslash S) .
$$

Portanto, a desigualdade acima deve ser uma igualdade e, pelo fato de que $\nu(A)<\infty$ (pois $A$ é limitado e $\nu \in \mathcal{M}$, logo é finita nos limitados), as duas desigualdades anteriores também devem ser igualdades. Em particular, $\nu_{\mu}(S)=\nu(B \cap S)$, estando provada a afirmação no caso em que $S$ é boreliano.

b) Caso geral: $S$ é um subconjunto arbitrário de $A$. Como $\nu$ e $\mu$ são Borel-regular, existe $T^{\prime} \in \mathscr{B}_{X}$ tal que

$$
S \subset T^{\prime}, \quad \mu(S)=\mu\left(T^{\prime}\right), \quad \nu(S)=\nu\left(T^{\prime}\right) .
$$

Seja $T:=A \cap T^{\prime} \in \mathscr{B}_{X}$. Temos $S \subset T \subset A$ e

$$
\mu(S) \leq \mu(T)=\mu\left(A \cap T^{\prime}\right) \leq \mu\left(T^{\prime}\right)=\mu(S) .
$$

Assim, $\mu(S)=\mu(T)$, e de forma análoga, temos $\nu(S)=\nu(T)$.

Como $S \subset A$, temos $\mu(S)<\infty$ e $\nu(S)<\infty$. Daí segue da Observação 1.6 que $T$ é tanto um $\mu$-envoltório como um $\nu$-envoltório de $S$. Então, para todo conjunto $\mu$-mensurável $E$, temos $\mu(S \cap E)=\mu(T \cap E)$ e, para todo $E \nu$-mensurável, tem-se $\nu(S \cap E)=\nu(T \cap E)$. Em particular, para todo $E \in \mathscr{B}_{X}, \mu(T \backslash E)=0$ se, e somente se, $\mu(S \backslash E)=0$, e daí segue

$$
\begin{aligned}
\nu_{\mu}(S) & =\inf \left\{\nu(E) \mid E \in \mathscr{B}_{X}, \mu(S \backslash E)=0\right\}= \\
& =\inf \left\{\nu(E) \mid E \in \mathscr{B}_{X}, \mu(T \backslash E)=0\right\}= \\
& =\nu_{\mu}(T) .
\end{aligned}
$$


Por outro lado, por a) e pelo fato de que $T$ é um $\nu$-envoltório de $S$, temos

$$
\nu_{\mu}(T)=\nu(T \cap B)=\nu(S \cap B) .
$$

Logo, $\nu_{\mu}(S)=\nu(B \cap S)$, como queríamos.

4) Seja $\left(A_{n}\right)_{n \in \mathbb{N}}$ uma sequência disjunta de borelianos limitados tal que $\dot{\bigcup}_{n \in \mathbb{N}} A_{n}=X$. Segue da parte 2) que, para cada $n \in \mathbb{N}, A_{n}$ contém um $B_{n} \in \mathscr{B}_{X}$, tal que $\mu\left(A_{n} \backslash B_{n}\right)=0$ e $\nu_{\mu}\left(A_{n}\right)=\nu\left(B_{n}\right)$. Da parte 3), tem-se, para todo $n \in \mathbb{N}, \nu_{\mu}\left\llcorner A_{n}=\nu\left\llcorner B_{n}\right.\right.$.

Daí, pondo $B:=\dot{\bigcup}_{n \in \mathbb{N}} B_{n} \in \mathscr{B}_{X}$, temos

$$
\nu_{\mu}=\sum_{n \in \mathbb{N}} \nu_{\mu}\left\llcorner A_{n}=\sum_{n \in \mathbb{N}} \nu\left\llcorner B_{n}=\nu\llcorner B\right.\right.
$$

Além disso, $\mu(X \backslash B)=\sum_{n \in \mathbb{N}} \mu\left(A_{n} \backslash B_{n}\right)=0$.

5) Se $A \subset X$ e $\mu(A)=0$, para $E=\emptyset \in \mathscr{B}_{X}$ tem-se $\mu(A \backslash E)=\mu(A)=0$, logo $\nu_{\mu}(A) \leq \nu(E)=$ $\nu(\emptyset)=0$, donde se conclui que $\nu_{\mu} \ll \mu$.

6) Finalmente, se $\nu \ll \mu$, tomando $B$ como em 4), tem-se $\nu\left\llcorner B^{c}=0\right.$, pois $\mu\left(B^{c}\right)=0$. Logo, $\nu=\nu\left\llcorner B+\nu\left\llcorner B^{c}=\nu\left\llcorner B=\nu_{\mu}\right.\right.\right.$. Reciprocamente, se $\nu=\nu_{\mu}$, então $\nu \ll \mu$ pela parte 5).

Observação 3.4. O Teorema 3.3 fornece a Decomposição de Lebesgue de $\nu$ com respeito a $\mu$, i.e. a parte absolutamente contínua é $\nu_{\mu}=\nu\left\llcorner B\right.$ e a parte singular é $\nu\left\llcorner B^{c}\right.$. A existência desta decomposição também pode ser obtida a partir do Lema 3.36 em [Ter19, pag. 74].

Definição 3.5 ( $V$-Derivada). Seja $\nu \in \mathcal{M}$. Com a notação da Definição 2.31 sobre limites ao longo de coberturas, para cada $x \in X$ definimos

$$
\underline{\mathrm{D}}(\nu, \mu, V, x):=(V) \liminf _{x} \frac{\nu}{\mu}, \quad \text { e } \quad \overline{\mathrm{D}}(\nu, \mu, V, x):=(V) \limsup _{x} \frac{\nu}{\mu} .
$$

Nos pontos onde os dois limites acima coincidem, definiremos a $V$-derivada de $\nu$ como

$$
\mathrm{D}(\nu, \mu, V, x):=\overline{\mathrm{D}}(\nu, \mu, V, x)=\underline{\mathrm{D}}(\nu, \mu, V, x) .
$$

Consideraremos $S \in V(X)$ tal que $\mu(S)=0$ no domínio de $\nu / \mu$, adotando, por convenção aritmética da reta estendida, $0 / 0:=0$ e, se $c>0, c / 0:=\infty$. Como as medidas são finitas nos conjuntos limitados, para o cômputo dos $(V) \limsup$ ou $(V) \lim$ inf basta considerar os conjuntos em $V(X)$ com medidas finitas.

Lema 3.6. Se $\alpha \in \mathcal{M}, \beta \in \mathcal{M}, 0<c<\infty$ e $A \subset\{x \mid \underline{\mathrm{D}}(\alpha, \beta, V, x)<c\}$, então $\alpha_{\mu}(A) \leq c \beta_{\mu}(A)$.

Demonstração. Fixe $\epsilon>0$. Pela definição de $\beta_{\mu}$, existe $E \in \mathscr{B}_{X} \operatorname{com} \mu(A \backslash E)=0$ tal que

$$
\beta(E) \leq \beta_{\mu}(A)+\frac{\epsilon}{2} .
$$

Agora, usando a hipótese $\beta \in \mathcal{M}$, existe uma sequência $\left(V_{i}\right)_{i \in \mathbb{N}}$ de conjuntos abertos com medida $\beta$ finita tal que $E \subset \bigcup_{i \in \mathbb{N}} V_{i}$. Então, pelo Teorema 1.16, $E$ está contido num aberto $W$ para o qual

$$
\beta(W \backslash E)<\frac{\epsilon}{2} .
$$

Daí,

$$
\beta(W)=\beta(E)+\beta(W \backslash E) \leq \beta_{\mu}(A)+\epsilon .
$$


Note que $A \backslash W \subset A \backslash E$, $\operatorname{logo} \mu(A \backslash W) \leq \mu(A \backslash E)=0$. E, como $\alpha \in \mathcal{M}$, pelo Teorema 3.3 temos $\alpha_{\mu} \ll \mu$, portanto $\alpha_{\mu}(A \backslash W)=0$.

Definamos

$$
C:=V \cap\left\{(x, S) \mid S \subset W, \frac{\alpha(S)}{\beta(S)}<c\right\} .
$$

Dado $x \in A \cap W$, o fato de $(V) \liminf _{x} \frac{\alpha}{\beta}<c$ garante que a existência de $\epsilon_{0}>0$ tal que, para $0<\epsilon<\epsilon_{0}$, tem-se

$$
0 \leq \inf \left\{\frac{\alpha(S)}{\beta(S)} \mid(x, S) \in V, \operatorname{diam} S \leq \epsilon\right\}<c .
$$

Portanto, se $0<\epsilon<\epsilon_{0}$, existe $S \in C$ com diam $S \leq \epsilon$, o que mostra que $C$ é fina em cada ponto de $A \cap W$. Note que, para todo $S \in C(A \cap W)$ tem-se $\frac{\alpha(S)}{\beta(S)}<c, \operatorname{logo} \alpha(S) \leq c \beta(S)$.

Assim, como $V$ é uma $\mu$-relação de Vitali, $C(A \cap W)$ tem uma subfamília enumerável disjunta $\mathcal{G}$ cobrindo $\mu$-q.t. $A \cap W$ i.e. $\mu((A \cap W) \backslash \bigcup \mathcal{G})=0$. Logo, lembrando que $\alpha_{\mu}(A \backslash W)=0$, segue

$$
\alpha_{\mu}(A)=\alpha_{\mu}(A \cap W) \leq \alpha(\bigcup \mathcal{G})=\sum_{S \in \mathcal{G}} \alpha(S) \leq c \sum_{S \in \mathcal{G}} \beta(S) \leq c \beta(W) \leq c\left(\beta_{\mu}(A)+\epsilon\right)
$$

Como isso vale para todo $\epsilon$ tal que $0<\epsilon<\epsilon_{0}$, temos $\alpha_{\mu}(A) \leq c \beta_{\mu}(A)$, como queríamos.

Corolário 3.7. Se $\nu \in \mathcal{M}$ e $0<c<\infty$ então

i) $A \subset\{x \mid \underline{\mathrm{D}}(\nu, \mu, V, x)<c\}$ implica $\nu_{\mu}(A) \leq c \mu(A)$,

ii) $A \subset\{x \mid \overline{\mathrm{D}}(\nu, \mu, V, x)>c\}$ implica $\nu_{\mu}(A) \geq c \mu(A)$.

Demonstração. Pelo Teorema 3.3, note que $\mu_{\mu}=\mu$, pois $\mu \ll \mu$ i.e. $\mu$ é absolutamente contínua com respeito a si mesma. O item $i)$ é consquência do Lema 3.6 aplicado para $A \subset\{x \mid \underline{\mathrm{D}}(\nu, \mu, V, x)<c\}$, o que acarreta

$$
\nu_{\mu}(A) \leq c \mu_{\mu}(A)=c \mu(A) .
$$

Agora, para o item $i i)$, o fato de que $(V) \lim \sup _{x} \frac{\nu}{\mu}>c$ garante a existência de $\epsilon_{0}>0$ tal que, para $0<\epsilon<\epsilon_{0}$, tem-se

$$
\sup \left\{\frac{\nu(S)}{\mu(S)} \mid(x, S) \in V, \operatorname{diam} S \leq \epsilon\right\}>c .
$$

Isto implica que para $0<\epsilon<\epsilon_{0}$,

$$
0 \leq \inf \left\{\frac{\mu(S)}{\nu(S)} \mid(x, S) \in V, \operatorname{diam} S \leq \epsilon\right\}<\frac{1}{c} .
$$

Logo,

$$
\underline{\mathrm{D}}(\mu, \nu, V, x)=(V) \liminf _{x} \frac{\mu}{\nu} \leq \frac{1}{c} .
$$

Daí, pelo Lema 3.6, temos $\mu(A)=\mu_{\mu}(A) \leq c^{-1} \nu_{\mu}(A)$, donde $c \mu(A) \leq \nu_{\mu}(A)$.

Teorema 3.8. Se $\nu \in \mathcal{M}$, então $0 \leq \mathrm{D}(\nu, \mu, V, x)<\infty$ para $\mu$-q.t. $x \in X$.

Demonstração.

1) Definamos

$$
C:=V \cap\{(x, S) \mid \mu(S)=0\} \quad \text { e } \quad P:=\{x \mid C \text { é fina em } x\} .
$$


Pelo fato de que $V$ é uma $\mu$-relação de Vitali, temos que $C(P)$ admite uma subfamília enumerável disjunta $\mathcal{G}$ cobrindo $\mu$-q.t. $P$, i.e. $\mu(P \backslash \bigcup \mathcal{G})=0$. Então encontramos que

$$
\mu(P)=\mu(P \cap \bigcup \mathcal{G})+\mu(P \backslash \bigcup \mathcal{G}) \leq \mu(\bigcup \mathcal{G})=\sum_{S \in \mathcal{G}} \mu(S)=0
$$

2) Seja $Q:=\{x \mid \overline{\mathrm{D}}(\nu, \mu, V, x)=\infty\}$. Note que, para cada $c>0, Q \subset\{x \mid \overline{\mathrm{D}}(\nu, \mu, V, x)>c\}$. Para qualquer subconjunto limitado $A$ de $Q$, pelo Corolário 3.7

$$
\mu(A) \leq \frac{1}{c} \nu_{\mu}(A)<\infty .
$$

Assim, tomando $c \rightarrow \infty$, conclui-se que $\mu(A)=0$. Podemos inferir que $\mu(Q)=0$. De fato, como $X$ é união enumerável de uma família disjunta $\left(A_{n}\right)_{n \in \mathbb{N}}$ de borelianos limitados, temos $Q=\bigcup_{n \in \mathbb{N}}\left(Q \cap A_{n}\right)$. Para cada $n, Q \cap A_{n}$ é um subconjunto limitado de $Q$, logo $\mu\left(Q \cap A_{n}\right)=0$, donde $\mu(Q) \leq \sum_{n \in \mathbb{N}} \mu\left(Q \cap A_{n}\right)=0$.

3) Agora, para cada $0<a<b$, definamos

$$
R(a, b):=\{x \mid \underline{\mathrm{D}}(\nu, \mu, V, x)<a<b<\overline{\mathrm{D}}(\nu, \mu, V, x)\} .
$$

Segue do Corolário 3.7 que, para qualquer subconjunto limitado $A$ de $R(a, b)$,

$$
b \mu(A) \leq \nu_{\mu}(A) \leq a \mu(A)<\infty,
$$

o que implica, tendo em vista que $a<b$ e $\mu(A)<\infty$, que $\mu(A)=0$. De forma análoga a 2 ), conclui-se que $\mu(R(a, b))=0$.

Finalmente, note que o conjunto dos pontos onde a derivada deixa de existir e é finita está contido na união de $P, Q$ e dos conjuntos $R(a, b)$ correspondendo a todos os racionais $a<b$, i.e. está contido numa união enumerável de conjuntos $\mu$-nulos, a qual é $\mu$-nula. Consequentemente $\mathrm{D}(\nu, \mu, V, x)$ existe e é finita em $\mu$-q.t. $x \in X$, como queríamos.

Lema 3.9. Se $\nu \in \mathcal{M}$, então $\mathrm{D}(\nu, \mu, V, \cdot)$ é $\mu$-mensurável.

Demonstração. Sejam $0<a<b<\infty$ e $A, B$ conjuntos limitados tais que

$$
A \subset P:=\{x \mid \mathrm{D}(\nu, \mu, V, x)<a\}, \quad \text { e } \quad B \subset Q:=\{x \mid \mathrm{D}(\nu, \mu, V, x)>b\} .
$$

1) Como $\mu, \nu_{\mu} \in \mathcal{M}$, pela Borel-regularidade existem borelianos $A^{\prime}$ e $B^{\prime}$ tais que

$$
\begin{array}{lll}
A \subset A^{\prime}, & \mu(A)=\mu\left(A^{\prime}\right), & \nu_{\mu}(A)=\nu_{\mu}\left(A^{\prime}\right) \\
B \subset B^{\prime}, & \mu(B)=\mu\left(B^{\prime}\right), & \nu_{\mu}(B)=\nu_{\mu}\left(B^{\prime}\right) .
\end{array}
$$

Note que, como $A$ e $B$ têm medidas $\mu$ e $\nu_{\mu}$ finitas (pois são limitados), segue da Observação 1.6 que $A^{\prime}$ é um envoltório de $A$ e $B^{\prime}$ um envoltório de $B$, ambos com respeito a $\mu$ e $\nu_{\mu}$. Daí, como $B^{\prime} \in \mathscr{B}_{X}$, segue

$$
\mu\left(A^{\prime} \cap B^{\prime}\right)=\mu\left(A \cap B^{\prime}\right) \quad \text { e } \quad \nu_{\mu}\left(A^{\prime} \cap B^{\prime}\right)=\nu_{\mu}\left(A \cap B^{\prime}\right) .
$$

Analogamente, como $A^{\prime} \in \mathscr{B}_{X}$, segue

$$
\mu\left(A^{\prime} \cap B^{\prime}\right)=\mu\left(A^{\prime} \cap B\right) \quad \text { e } \quad \nu_{\mu}\left(A^{\prime} \cap B^{\prime}\right)=\nu_{\mu}\left(A^{\prime} \cap B\right) .
$$


Do Corolário 3.7, para $A \cap B^{\prime} \subset P$ e $A^{\prime} \cap B \subset Q$ vemos que

$$
\begin{aligned}
& \nu_{\mu}\left(A \cap B^{\prime}\right) \leq a \mu\left(A \cap B^{\prime}\right), \\
& \nu_{\mu}\left(A^{\prime} \cap B\right) \geq b \mu\left(A^{\prime} \cap B\right) .
\end{aligned}
$$

Portanto, $b \mu\left(A^{\prime} \cap B^{\prime}\right) \leq a \mu\left(A^{\prime} \cap B^{\prime}\right)$. Tendo em vista que $a<b$ e que $\mu\left(A^{\prime} \cap B^{\prime}\right) \leq \mu\left(A^{\prime}\right)=$ $\mu(A)<\infty$, segue $\mu\left(A^{\prime} \cap B^{\prime}\right)=0$.

Agora,

$$
\mu(A \cup B)=\mu\left((A \cup B) \cap\left(A^{\prime} \cup B^{\prime}\right)\right)=\mu\left\llcorner(A \cup B)\left(A^{\prime} \cup B^{\prime}\right)\right.
$$

e, como $\mu\llcorner(A \cup B)$ é uma medida boreliana finita, segue

$$
\begin{aligned}
\mu(A \cup B) & =\mu\left\llcorner(A \cup B)\left(A^{\prime}\right)+\mu\llcorner(A \cup B)\left(B^{\prime}\right)-\underbrace{\mu\left\llcorner(A \cup B)\left(A^{\prime} \cap B^{\prime}\right)\right.}_{\leq \mu\left(A^{\prime} \cap B^{\prime}\right)=0} \geq\right. \\
& \geq \mu\left(A^{\prime}\right)+\mu\left(B^{\prime}\right)=\mu(A)+\mu(B) .
\end{aligned}
$$

Portanto, $\mu(A \cup B)=\mu(A)+\mu(B)$.

2) Como $X$ é união enumerável de uma família disjunta $\left(E_{n}\right)_{n \in \mathbb{N}}$ de borelianos limitados, todo $T \subset X$ pode ser escrito como $T=\dot{\bigcup}_{n \in \mathbb{N}}\left(T \cap E_{n}\right)$, donde $T \cap P=\dot{\bigcup}_{n \in \mathbb{N}}\left(T \cap P \cap E_{n}\right)$ e $T \cap Q=\bigcup_{n \in \mathbb{N}}\left(T \cap Q \cap E_{n}\right)$.

Para cada $n \in \mathbb{N}, T \cap P \cap E_{n}$ e $T \cap Q \cap E_{n}$ são subconjuntos limitados de $P$ e $Q$, respectivamente; daí, pelo item 1), para cada $n \in \mathbb{N}$,

$$
\mu\left(T \cap P \cap E_{n}\right)+\mu\left(T \cap Q \cap E_{n}\right)=\mu\left(\left(T \cap P \cap E_{n}\right) \cup\left(T \cap Q \cap E_{n}\right)\right) \leq \mu\left(T \cap E_{n}\right) .
$$

Então,

$$
\begin{aligned}
\mu(T \cap P)+\mu(T \cap Q) & \leq \sum_{n \in \mathbb{N}} \mu\left(T \cap P \cap E_{n}\right)+\sum_{n \in \mathbb{N}} \mu\left(T \cap Q \cap E_{n}\right) \leq \sum_{n \in \mathbb{N}} \mu\left(T \cap E_{n}\right)= \\
& =\sum_{n \in \mathbb{N}} \mu\left\llcorner T\left(E_{n}\right)=\mu\llcorner T(X)=\mu(T) .\right.
\end{aligned}
$$

Como $a, b \in \mathbb{R}$ tais que $0<a<b<\infty$ foram tomados arbitrariamente, segue da Proposição 1.24 que $\mathrm{D}(\nu, \mu, V, \cdot)$ é $\mu$-mensurável.

Teorema 3.10. Se $\nu \in \mathcal{M}$ e A é $\mu$-mensurável, então A é $\nu_{\mu}$-mensurável e

$$
\nu_{\mu}(A)=\int_{A} \mathrm{D}(\nu, \mu, V, x) d \mu(x) .
$$

Demonstração. Pela Borel-regularidade e pela $\sigma$-finitude de $\mu$ (note que $\mu$ é $\sigma$-finita porque, por hipótese, é finita nas partes limitadas de $X)$, existe $B \in \mathscr{B}_{X}$ tal que $A \subset B$ e $\mu(B \backslash A)=0$. Como $\nu_{\mu} \ll \mu$, temos $\nu_{\mu}(B \backslash A)=0$; portanto, $A$ é $\nu_{\mu}$-mensurável.

Sejam

$$
Z:=\{x \mid \mathrm{D}(\nu, \mu, V, x)=0\} \quad \text { e } \quad W:=\{x \mid \mathrm{D}(\nu, \mu, V, x)=\infty\} .
$$

Pelo Lema 3.9, os conjuntos $Z$ e $W$ são $\mu$-mensuráveis. E, pelo Teorema $3.8, \mu(W)=0, \operatorname{logo}$ $\nu_{\mu}(W)=0$. Por outro lado, para cada $c>0, Z \subset\{x \mid \underline{\mathrm{D}}(\nu, \mu, V, x)<c\}$. Portanto, para todo 
subconjunto limitado $A^{\prime}$ de $Z$ e para todo $c>0$, segue do Corolário 3.7 que

$$
\nu_{\mu}\left(A^{\prime}\right) \leq c \mu\left(A^{\prime}\right)<\infty \text {. }
$$

Logo, fazendo $c \rightarrow 0$, segue $\nu_{\mu}\left(A^{\prime}\right)=0$. Finalmente, como $Z$ pode ser escrito como união enumerável de limitados, por subaditividade enumerável conclui-se que $\nu_{\mu}(Z)=0$.

Fixe $1<t<\infty$. Definamos, para cada inteiro $n$,

$$
P_{n}:=A \cap\left\{x \mid t^{n} \leq \mathrm{D}(\nu, \mu, V, x)<t^{n+1}\right\} .
$$

Pelo Lema 3.9, os $P_{n}$ 's são $\mu$-mensuráveis e, portanto, como visto acima, $\nu_{\mu}$-mensuráveis. Além disso, $A \backslash(Z \cup W)=\dot{\bigcup}_{n \in \mathbb{Z}} P_{n}$ i.e. é a união enumerável dos conjuntos disjuntos $P_{n}$. Note que $\nu_{\mu}\left(A \backslash \dot{\cup}_{n \in \mathbb{Z}} P_{n}\right)=\nu_{\mu}(Z \cup W)=0$.

Do Corolário 3.7, segue

$$
\begin{aligned}
\nu_{\mu}(A) & =\nu_{\mu}\left(\bigcup_{n \in \mathbb{Z}} P_{n}\right)=\sum_{n \in \mathbb{Z}} \nu_{\mu}\left(P_{n}\right) \leq \sum_{n \in \mathbb{Z}} t^{n+1} \mu\left(P_{n}\right)=t \sum_{n \in \mathbb{Z}} t^{n} \mu\left(P_{n}\right) \leq \\
& \leq t \sum_{n \in \mathbb{Z}} \int_{P_{n}} \mathrm{D}(\nu, \mu, V, x) d \mu(x)=t \int_{A} \mathrm{D}(\nu, \mu, V, x) d \mu(x) .
\end{aligned}
$$

Por outro lado,

$$
\begin{aligned}
\nu_{\mu}(A) & =\sum_{n \in \mathbb{Z}} \nu_{\mu}\left(P_{n}\right) \geq \sum_{n \in \mathbb{Z}} t^{n} \mu\left(P_{n}\right)=t^{-1} \sum_{n \in \mathbb{Z}} t^{n+1} \mu\left(P_{n}\right) \\
& \geq t^{-1} \sum_{n \in \mathbb{Z}} \int_{P_{n}} \mathrm{D}(\nu, \mu, V, x) d \mu(x)=t^{-1} \int_{A} \mathrm{D}(\nu, \mu, V, x) d \mu(x) .
\end{aligned}
$$

Portanto, para todo $1<t<\infty, t^{-1} \int_{A} \mathrm{D}(\nu, \mu, V, x) d \mu(x) \leq \nu_{\mu}(A) \leq t \int_{A} \mathrm{D}(\nu, \mu, V, x) d \mu(x)$, Fazendo $t \rightarrow 1$, segue

$$
\nu_{\mu}(A)=\int_{A} \mathrm{D}(\nu, \mu, V, x) d \mu(x) .
$$

Teorema 3.11 (Teorema de diferenciação de Lebesgue). Seja $f$ uma função $\mu$-mensurável em $X$ com valores em $\overline{\mathbb{R}}$ tal que, para todo conjunto limitado $\mu$-mensurável $A$,

$$
\int_{A}|f| d \mu<\infty
$$

Então, para $\mu$-q.t. $x \in X$

$$
\text { (V) } \lim _{S \rightarrow x} \frac{1}{\mu(S)} \int_{S} f d \mu=f(x) .
$$

Demonstração. Suponhamos que $f$ é não negativa. Para todo $A \subset X$, definimos $\nu \operatorname{por}^{1}$

$$
\nu(A):=\int_{A}^{*} f d \mu .
$$

Pela Observação 1.28, $\nu$ é uma medida Borel-regular em $X$. Pela hipótese, $\nu$ é finita nos limitados de $X, \log o, \in \mathcal{M}$. É claro que $\nu \ll \mu$; portanto, pelo Teorema 3.3, $\nu=\nu_{\mu}$. Assim, do Teorema

\footnotetext{
${ }^{1}$ Equivalentemente, podemos definir $\nu$ por $A \mapsto \int_{A} f \mathrm{~d} \mu$ se $A \in \mathscr{B}_{X}$ e tomar a medida exterior induzida, i.e. $\forall E \subset X, \nu(E):=\inf \left\{\nu(A) \mid E \subset A \in \mathscr{B}_{X}\right\}$. As duas definições são equivalentes porque ambas resultam em medidas borelianas regulares que coincidem em $\mathscr{B}_{X}$.
} 
3.10, temos, para todo $A \mu$-mensurável,

$$
\int_{A} f(x) d \mu(x)=\nu(A)=\nu_{\mu}(A)=\int_{A} \mathrm{D}(\nu, \mu, V, x) d \mu(x) .
$$

e daí se conclui que, para $\mu$-q.t. $x \in X$,

$$
f(x)=\mathrm{D}(\nu, \mu, V, x)=(V) \lim _{S \rightarrow x} \frac{\nu(S)}{\mu(S)}=(V) \lim _{S \rightarrow x} \frac{1}{\mu(S)} \int_{S} f d \mu .
$$

Isso conclui a prova no caso que $f \geq 0$; no caso geral, podemos aplicar o anterior para $f^{+}$e $f^{-}$.

Corolário 3.12 (Pontos de Lebesgue). Seja $f$ uma função $\mu$-mensurável em $X$ com valores num espaço vetorial normado separável $Y$, e suponha que, para todo conjunto limitado $\mu$-mensurável $A$,

$$
\int_{A}|f| d \mu<\infty
$$

Então, para $\mu$-q.t $x \in X$,

$$
(V) \lim _{S \rightarrow x} \frac{1}{\mu(S)} \int_{S}|f(z)-f(x)| d \mu(z)=0
$$

Demonstração. A cada ponto $x \in X$ associaremos o conjunto $T(x)$ de todos os pontos $y \in Y$ para os quais

$$
(V) \lim _{S \rightarrow x} \frac{1}{\mu(S)} \int_{S}|f(z)-y| d \mu(z)=|f(x)-y| .
$$

Seja $C:=\left\{y_{i} \mid i \in \mathbb{N}\right\}$ um subconjunto denso enumerável de $Y$. Para cada $i \in \mathbb{N}$, o Teorema 3.11 aplicado a $\left|f-y_{i}\right|$ garante a existência de $P_{i} \subset X \mu$-nulo tal que $y_{i} \in T(x)$ se $x \in X \backslash P_{i}$. Tomemos $N:=P \cup \bigcup_{i \in \mathbb{N}} P_{i}$, onde $P$ é definido como na prova do Teorema 3.8. Então $N$ é $\mu$-nulo, pois é união enumerável de $\mu$-nulos. Seja $x \in X \backslash N$ e fixe $\epsilon>0$. Escolhamos $y_{i} \in C$ tal que $\left|f(x)-y_{i}\right|<\epsilon / 2$. Então, para todo $S \in V(\{x\})$,

$$
\begin{aligned}
\frac{1}{\mu(S)} \int_{S}|f(z)-f(x)| d \mu(z) & \leq \frac{1}{\mu(S)} \int_{S}\left(\left|f(z)-y_{i}\right|+\left|y_{i}-f(x)\right|\right) d \mu(z) \\
& \leq \frac{1}{\mu(S)} \int_{S}\left|f(z)-y_{i}\right| d \mu(z)+\left|y_{i}-f(x)\right|,
\end{aligned}
$$

de modo que

$$
\begin{aligned}
(V) \lim _{S \rightarrow x} \frac{1}{\mu(S)} \int_{S}|f(z)-f(x)| d \mu(z) & \leq(V) \lim _{S \rightarrow x} \frac{1}{\mu(S)} \int_{S}\left|f(z)-y_{i}\right| d \mu(z)+\left|y_{i}-f(x)\right| \\
& =2\left|f(z)-y_{i}\right|<\epsilon
\end{aligned}
$$

Como $\epsilon>0$ foi fixado arbitrariamente, conclui-se que (3.1) vale para todo $x$ no complementar do $\mu$-nulo $N$.

Definição 3.13. Com a notação do corolário anterior, o conjunto dos pontos onde (3.1) se verifica é chamado o $(\mu, V)$-conjunto Lebesgue de $f$.

Proposição 3.14. Seja $\nu \in \mathcal{M}$. Tem-se:

i) Para $\mu$-q.t. $x \in X$,

$$
\mathrm{D}\left(\nu_{\mu}, \mu, V, x\right)=\mathrm{D}(\nu, \mu, V, x) \quad e \quad \mathrm{D}\left(\nu-\nu_{\mu}, \mu, V, x\right)=0 .
$$


ii) Se $V_{1}$ e $V_{2}$ são duas $\mu$-relações Vitali, então, para $\mu$-q.t. $x \in X$,

$$
\mathrm{D}\left(\nu, \mu, V_{1}, x\right)=\mathrm{D}\left(\nu, \mu, V_{2}, x\right) .
$$

Demonstração. Note que $\nu_{\mu}=\left(\nu_{\mu}\right)_{\mu}$, pois $\nu_{\mu} \ll \mu$. Do Teorema 3.10 com $\nu_{\mu} \in \mathcal{M}$ no lugar de $\nu$, obtém-se, para todo $A \subset X \mu$-mensurável,

$$
\nu_{\mu}(A)=\int_{A} \mathrm{D}\left(\nu_{\mu}, \mu, V, x\right) d \mu(x) .
$$

Por outro lado, pelo Teorema 3.10 aplicado a $\nu$, o que vai acima também coincide com $\int_{A} \mathrm{D}(\nu, \mu, V, x) d \mu(x)$. Pela arbitrariedade do $A \subset X \mu$-mensurável tomado, conclui-se que, para $\mu$-q.t. $x \in X$,

$$
\mathrm{D}\left(\nu_{\mu}, \mu, V, x\right)=\mathrm{D}(\nu, \mu, V, x) .
$$

Portanto, para $\mu$-q.t. $x \in X$,

$$
\mathrm{D}\left(\nu-\nu_{\mu}, \mu, V, x\right)=0 .
$$

Finalmente, como $\nu_{\mu}$ foi definida sem referência a $V$, a igualdade enunciada no Teorema 3.10 vale para todo $A \mu$-mensurável com $V_{1}$ ou $V_{2}$ no lugar de $V$. Isso implica, para $\mu$-q.t. $x \in X$,

$$
\mathrm{D}\left(\nu, \mu, V_{1}, x\right)=\mathrm{D}\left(\nu, \mu, V_{2}, x\right) .
$$

\subsection{Densidade e Continuidade aproximada}

Nesta seção, estudamos a noção de densidade associada a uma medida $\mu \in \mathcal{M}$. Na Definição 3.18, por meio da referida noção, introduziremos os conceitos de limite e continuidade aproximados.

Observação 3.15. Note que, se $\mu \in \mathcal{M}$, todo $A \subset X$ tem um $\mu$-envoltório boreliano. De fato, se $A$ for limitado (portanto, $\mu(A)<\infty$ ), pela Borel-regularidade de $\mu$ existe $B \in \mathscr{B}_{X}$ tal que $A \subset B$ e $\mu(A)=\mu(B)<\infty$; daí, pela Observação 1.6, o boreliano $B$ é um $\mu$-envoltório de $A$.

No caso geral, podemos escrever $A$ como união de uma sequência crescente $\left(A_{n}\right)_{n \in \mathbb{N}}$ de limitados e, para cada $n \in \mathbb{N}$, o caso já provado garante a existência de um envoltório boreliano $B_{n}$ para $A_{n}$. Substituindo-se, para cada $n \in \mathbb{N}, B_{n}$ por $\bigcup_{i=1}^{n} B_{i}$ (que também será um envoltório boreliano de $A_{n}$ ), podemos supor que a sequência $\left(B_{n}\right)_{n \in \mathbb{N}}$ também é crescente. Daí $B:=\bigcup_{n \in \mathbb{N}} B_{n} \in \mathscr{B}_{X}$ é um envoltório boreliano de $A$, pois, para todo $T \in \sigma(\mu), \mu(T \cap B)=\lim \mu\left(T \cap B_{n}\right)=\lim \mu\left(T \cap A_{n}\right) \leq \mu(T \cap A)$.

Além disso, se $B \in \mathscr{B}_{X}$, segue do Teorema 1.18 e da Observação 1.19 que $\mu\llcorner B \in \mathcal{M}$.

Teorema 3.16. Se $A \subset X e$

$$
P:=\left\{x \mid(V) \lim _{S \rightarrow x} \frac{\mu(S \cap A)}{\mu(S)}=1\right\}, \quad Q:=\left\{x \mid(V) \lim _{S \rightarrow x} \frac{\mu(S \backslash A)}{\mu(S)}=0\right\} .
$$

Então $P$ e $Q$ são $\mu$-mensuráveis,

$$
\begin{aligned}
& \mu(A \backslash P)=0, \quad A \cup P \text { é um } \mu \text {-envoltório de } A, \\
& \mu(Q \backslash A)=0, \quad(A \cap Q)^{c} \text { é um } \mu \text {-envoltório de } A^{c} .
\end{aligned}
$$

Além disso, a $\mu$-mensurabilidade de A é equivalente a cada uma das duas condições:

$$
\mu(P \backslash A)=0 ; \quad \mu(A \backslash Q)=0 .
$$


Demonstração. Pela Observação 3.15, todo $A \subset X$ tem um $\mu$-envoltório boreliano.

1. Seja $B$ um $\mu$-envoltório boreliano de $A$. Então $B \in \mathscr{B}_{X}, A \subset B$ e, para todo conjunto $\mu$ mensurável $S$, em particular para $S \in V(X)$, temos $\mu(S \cap A)=\mu(S \cap B)=\mu\llcorner B(S)$. Logo, para todo $x \in X$,

$$
(V) \limsup _{S \rightarrow x} \frac{\mu(S \cap A)}{\mu(S)}=(V) \limsup _{S \rightarrow x} \frac{\mu\llcorner B(S)}{\mu(S)}=\overline{\mathrm{D}}(\mu\llcorner B, \mu, V, x),
$$

e o mesmo vale para $(V) \liminf$. Então, como $\mu\llcorner B \in \mathcal{M}$ temos que

$$
P=\{x \mid \mathrm{D}(\mu\llcorner B, \mu, V, x)=1\}
$$

é $\mu$-mensurável pelo Lema 3.9. Aplicando o Teorema 3.11 para $f=\chi_{B}$, conclui-se que $\mu(B \backslash P)=0$ e $\mu(P \backslash B)=0$.

Como $A \subset B$ temos $A \backslash P \subset B \backslash P, \log o \mu(A \backslash P)=0$. Além disso, como $P$ é $\mu$-mensurável e $(A \cup P) \backslash P=A \backslash P$ é $\mu$-nulo, conclui-se que $A \cup P$ é $\mu$-mensurável. Por outro lado, $A \subset A \cup P$ e $A \cup P \subset B \cup P=B \cup(P \backslash B)$.

Daí, para todo $T \mu$-mensurável, temos $\mu(T \cap A) \leq \mu(T \cap(A \cup P))$ e

$$
\begin{aligned}
\mu(T \cap(A \cup P)) & \leq \mu(T \cap(B \cup(P \backslash B))) \leq \mu(T \cap B)+\mu(T \cap(P \backslash B)) \leq \\
& \leq \mu(T \cap B)+\mu(P \backslash B)=\mu(T \cap B)=\mu(T \cap A),
\end{aligned}
$$

onde a última igualdade vale porque $B$ é $\mu$-envoltório de $A$. Portanto, $A \cup P$ é um $\mu$-envoltório de $A$.

2. De forma análoga, seja $C$ um $\mu$-envoltório boreliano de $A^{c}$. Então $C \in \mathscr{B}_{X}, A^{c} \subset C$ e, para todo $S \subset X \mu$-mensurável, em particular para $S \in V(X)$, temos $\mu(S \backslash A)=\mu(S \cap C)$. Logo, para todo $x \in X$,

$$
(V) \limsup _{S \rightarrow x} \frac{\mu(S \backslash A)}{\mu(S)}=(V) \limsup _{S \rightarrow x} \frac{\mu\llcorner C(S)}{\mu(S)}=\overline{\mathrm{D}}(\mu\llcorner C, \mu, V, x),
$$

e o mesmo vale para $(V) \lim$ inf. Então, como $\mu\llcorner C \in \mathcal{M}$, segue do Lema 3.9 que

$$
Q=\{x \mid \mathrm{D}(\mu\llcorner C, \mu, V, x)=0\}
$$

é $\mu$-mensurável. Aplicando o Teorema 3.11 para $f=\chi_{C}$, conclui-se que $\mu(Q \cap C)=0$ e $\mu\left((C \cup Q)^{c}\right)=0$.

Como $A^{c} \subset C$ temos $Q \backslash A=Q \cap A^{c} \subset Q \cap C$, portanto $\mu(Q \backslash A)=0$. Além disso, como $C$ é $\mu$-mensurável e $(A \cap Q)^{c} \backslash C \subset Q^{c} \backslash C=(Q \cup C)^{c}$ é $\mu$-nulo, conclui-se que $(A \cap Q)^{c}$ é $\mu$-mensurável. Por outro lado, como $A \cap Q \subset A$, temos

$$
A^{c} \subset(A \cap Q)^{c}=A^{c} \cup Q^{c} \subset C \cup Q^{c}=C \cup\left(C^{c} \cap Q^{c}\right)=C \cup(C \cup Q)^{c} .
$$

Daí, para todo $T \mu$-mensurável, temos

$$
\begin{aligned}
\mu\left(T \cap A^{c}\right) & \leq \mu\left(T \cap(A \cap Q)^{c}\right) \leq \mu\left(T \cap\left(C \cup(C \cup Q)^{c}\right)\right) \leq \mu(T \cap C)+\mu\left(T \cap(C \cup Q)^{c}\right) \leq \\
& \leq \mu(T \cap C)+\mu\left((C \cup Q)^{c}\right)=\mu(T \cap C)=\mu\left(T \cap A^{c}\right) .
\end{aligned}
$$

Portanto, $\mu\left(T \cap(A \cap Q)^{c}\right)=\mu\left(T \cap A^{c}\right)$, provando que $(A \cap Q)^{c}$ é um $\mu$-envoltório de $A^{c}$.

3. Agora, se $A$ for $\mu$-mensurável, como $A \cup P$ é um $\mu$-envoltório de $A$, temos

$$
\mu(P \backslash A)=\mu\left(P \cap A^{c}\right)=\mu\left((A \cup P) \cap A^{c}\right)=\mu\left(A \cap A^{c}\right)=0 .
$$


Por outro lado, como $(A \cap Q)^{c}$ é um $\mu$-envoltório de $A^{c}$, temos

$$
\mu(A \backslash Q)=\mu\left(A \cap Q^{c}\right)=\mu\left(A \cap\left(A^{c} \cup Q^{c}\right)\right)=\mu\left(A \cap(A \cap Q)^{c}\right)=\mu\left(A \cap A^{c}\right)=0 .
$$

Reciprocamente, suponha que $\mu(P \backslash A)=0$. Para todo $T \subset X$, temos

$$
\mu(T) \leq \mu(T \cap A)+\mu(T \backslash A) .
$$

Como $P$ é $\mu$-mensurável,

$$
\mu(T \cap A)=\mu((T \cap A) \cap P)+\mu((T \cap A) \backslash P) \leq \mu(T \cap P)+\mu(A \backslash P)=\mu(T \cap P),
$$

pois $\mu(A \backslash P)=0$. Por outro lado,

$$
\mu\left(T \cap A^{c}\right)=\mu\left(\left(T \cap A^{c}\right) \cap P\right)+\mu\left(\left(T \cap A^{c}\right) \cap P^{c}\right) \leq \mu\left(P \cap A^{c}\right)+\mu\left(T \cap P^{c}\right)=\mu\left(T \cap P^{c}\right),
$$

pois $\mu(P \backslash A)=0$.

Logo, para todo $T \subset X, \mu(T \cap A)+\mu\left(T \cap A^{c}\right) \leq \mu(T \cap P)+\mu\left(T \cap P^{c}\right)=\mu(T)$, donde a $\mu$-mensurabilidade de $A$.

De forma análoga se prova que, se $\mu(A \backslash Q)=0$, então $A$ é $\mu$-mensurável.

Definição 3.17 (Densidade). Para todo $A \subset X$ e $x \in X$, definimos a $(\mu, V)$-densidade de $A$ em $x$ por

$$
\Theta(\mu, A, x):=(V) \lim _{S \rightarrow x} \frac{\mu(S \cap A)}{\mu(S)},
$$

caso o limite exista.

Definição 3.18 (Limites aproximados). Sejam $Y$ um espaço topológico, $f: \operatorname{dom} f \subset X \rightarrow Y$ e $x \in X$. Um ponto $y \in Y$ é chamado de $(\mu, V)$-limite aproximado de $f$ em $x$ se, para toda vizinhança $W$ de $y \in Y$, o conjunto $X \backslash f^{-1}(W)$ tem $(\mu, V)$-densidade 0 em $x$.

Observação 3.19. Da Definição 3.18, no caso que $Y$ seja um espaço Hausdorff, pode haver no máximo um limite aproximado em $x$, o qual será denotado por

$$
(\mu, V) \text { ap } \lim _{x} f, \quad \text { ou } \quad(\mu, V) \text { ap } \lim _{z \rightarrow x} f(z) .
$$

De fato, se $Y$ for um espaço Hausdorff e a função $f$ tiver limites aproximados $y \neq y^{\prime}$ em $X$, teríamos dois abertos disjuntos $W$ e $W^{\prime}$ em $Y$ tais que $X \backslash f^{-1}(W)$ e $X \backslash f^{-1}\left(W^{\prime}\right)$ tem ambos densidade 0 em $x$. Como a união dos dois últimos conjuntos é $X$, conclui-se que $X$ tem densidade 0 em $x$, chegando-se a uma contradição.

Definição 3.20. Sejam $f: X \rightarrow \overline{\mathbb{R}}$ e $x \in X$. Definimos o limite superior aproximado de $x$,

$$
(\mu, V) \operatorname{ap} \limsup _{x} f \in \overline{\mathbb{R}}
$$

como sendo o ínfimo do conjunto de todos os números reais $t$ tal que $\{z \mid f(z)>t\}$ tem densidade 0 em $x$. Analogamente, definimos limite inferior aproximado de $x$,

$$
(\mu, V) \text { ap } \liminf _{x} f \in \overline{\mathbb{R}},
$$

como sendo o supremo do conjunto de todos os números reais $t$ tal que $\{z \mid f(z)<t\}$ tem densidade 0 em $x$. 
Definição 3.21. Dizemos que $f: X \rightarrow Y$ é $(\mu, V)$-aproximadamente contínua em $x$ se $x \in \operatorname{dom} f$ e

$$
(\mu, V) \text { ap } \lim _{x} f=f(x) .
$$

Segundo [Fed96], o Teorema de Lusin, descrito no Teorema 1.25, mostra uma conexão global entre mensurabilidade e continuidade; o seguinte teorema localiza essa relação.

Teorema 3.22. Seja $f$ uma função levando $\mu$-q.t. $X$ num espaço métrico separável $Y$. Então $f$ é $\mu$-mensurável se, e somente se $f$ é $(\mu, V)$-aproximadamente contínua em $\mu$-q.t. ponto de $X$.

Demonstração. Podemos decompor $X$ numa união enumerável disjunta de uma família de borelianos $\left(E_{n}\right)_{n \in \mathbb{N}}$ tal que, para todo $n \in \mathbb{N}, \mu\left(E_{n}\right)<\infty$. Para cada $n, k \in \mathbb{N}$, pelo Teorema de Lusin 1.25 podemos tomar um fechado $C_{n, k}$ em $X$ tal que $C_{n, k} \subset E_{n}, \mu\left(E_{n} \backslash C_{n, k}\right)<1 / k$ e $\left.f\right|_{C_{n, k}}$ contínua. Tomando-se uma enumeração $\phi: \mathbb{N} \rightarrow \mathbb{N} \times \mathbb{N}$ e pondo, para todo $n \in \mathbb{N}, C_{n}:=C_{\phi(n)}$, obtém-se uma sequência $\left(C_{n}\right)_{n \in \mathbb{N}}$ de fechados em $X$ tal que $\mu\left(X \backslash \bigcup_{n \in \mathbb{N}} C_{n}\right)=0$ e, para todo $n \in \mathbb{N},\left.f\right|_{C_{n}}$ é contínua.

Além disso, o Teorema 3.16 implica que, para todo $n \in \mathbb{N}$ e para $\mu$-q.t. $x \in C_{n}$,

$$
(V) \lim _{S \rightarrow x} \frac{\mu\left(S \backslash C_{n}\right)}{\mu(S)}=0
$$

para um tal $x, f$ é $(\mu, V)$-aproximadamente contínua em $x$, o que mostra que $f$ é $(\mu, V)$-aproximadamente contínua $\mu$-q.s. em $X$.

Reciprocamente, se $f$ é $(\mu, V)$-aproximadamente continua em $\mu$-q.t. ponto de $X$, e se $W$ é um subconjunto aberto em $Y$, temos, para todo $x \in f^{-1}(W)$,

$$
(V) \lim _{S \rightarrow x} \frac{\mu\left(S \backslash f^{-1}(W)\right)}{\mu(S)}=0,
$$

o que garante a $\mu$-mensurabilidade de $f^{-1}(W)$ pelo Teorema 3.16. Portanto, pela arbitrariedade do aberto $W \subset Y$ tomado, conclui-se que $f$ é $\mu$-mensurável.

\subsection{Derivação de medidas usando bolas centralizadas}

Proposição 3.23. Suponha $J \subset\{r \mid 0<r<\infty\}$ aberto. Sejam $\alpha, \beta$ medidas em $X$, com $\beta \in \mathcal{M}$. Então $\{x \mid \alpha(\mathbb{B}(x, r))>\beta(\mathbb{B}(x, r))$, para algum $r \in J\}$ é aberto.

Demonstração. De fato, suponha que $\alpha(\mathbb{B}(a, r))>\beta(\mathbb{B}(a, r))$, com $r \in J$. Como $\beta \in \mathcal{M}$, é finita nos limitados de $X$, a continuidade para baixo de $\beta$ permite concluir que $\beta(\mathbb{B}(x, \cdot))$ é contínua à direita. Assim, existe $\epsilon>0$ para o qual $r+\epsilon \in J$ e $\alpha(\mathbb{B}(a, r))>\beta(\mathbb{B}(a, r+2 \epsilon))$. Portanto, $x \in \mathbb{B}(a, r)$ implica que

$$
\alpha(\mathbb{B}(x, r+\epsilon)) \geq \alpha(\mathbb{B}(a, r))>\beta(\mathbb{B}(a, r+2 \epsilon)) \geq \beta(\mathbb{B}(x, r+\epsilon)) .
$$

Corolário 3.24. Sejam $\mu \in \mathcal{M}$ e $0<t<\infty$.

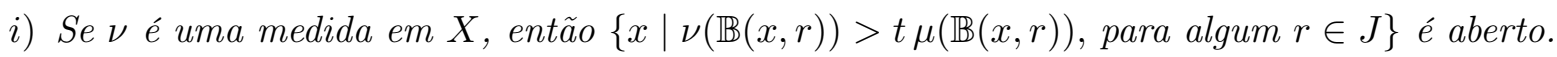

ii) Se $\nu \in \mathcal{M}$, então $\{x \mid \nu(\mathbb{B}(x, r))<t \mu(\mathbb{B}(x, r))$, para algum $r \in J\}$ é aberto.

Demonstração. O item $i$ ) se obtém aplicando a Proposição 3.23 para $\alpha=\nu$ e $\beta=t \mu \in \mathcal{M}$. Analogamente obtemos ii) aplicando de novo a Proposição 3.23 para $\alpha=\mu$ e $\beta=t^{-1} \nu \in \mathcal{M}$. 
Observação 3.25. Considere a relação de cobertura $V=\{(x, \mathbb{B}(x, r)) \mid x \in X, 0<r<\infty\}$. Dados $a, b \in \mathbb{R}, x \in X$ e $f$ uma função definida em $V(\{x\})=\{\mathbb{B}(x, r) \mid r>0\}$ a valores em $\overline{\mathbb{R}}$, tem-se:

a) Se $g(r):=f(\mathbb{B}(x, r))$ :

- $\lim \sup _{r \rightarrow 0} g(r) \leq a$ se, e somente se, para todo $\epsilon>0$, existe $r_{0}>0$ tal que $g(r) \leq a+\epsilon$ sempre que $0<r \leq r_{0}$.

- $\lim \sup _{r \rightarrow 0} g(r) \geq a$ se, e somente se, para todo $r_{0}>0$ e todo $\epsilon>0$, existe $0<r<r_{0}$ tal que $g(r) \geq a-\epsilon$, i.e. se, e somente se, para todo $\epsilon>0$ existe $r_{n} \downarrow 0$ tal que $g\left(r_{n}\right) \geq a-\epsilon$ para todo $n \in \mathbb{N}$.

- Caracterizações análogas valem para $\liminf _{r \rightarrow 0} g(r)$ e também para $(V) \lim \sup _{S \rightarrow x} f(S)$ ou $(V) \liminf _{S \rightarrow x} f(S)$.

b) Suponha que exista $\delta>0$ tal que $f(S)<a$ sempre que diam $S<\delta$. Então, se $0<2 r<\delta$, tem-se diam $\mathbb{B}(x, r) \leq 2 r<\delta$, portanto $f(\mathbb{B}(x, r))<a$. Respectivamente para $f(S)>b$, temos $f(\mathbb{B}(x, r))>b$.

c) Suponha que exista $\delta>0$ tal que $f(\mathbb{B}(x, r))<a$ sempre que $0<r \leq \delta$. Então, se $B \in V(\{x\})$ e diam $B \leq \delta$, tem-se $B \subset \mathbb{B}(x, \delta)$, logo existe $r \leq \delta$ tal que $B=\mathbb{B}(x, r)$, daí $f(B)<a$. Analogamente com $f(\mathbb{B}(x, r))>b$, temos $f(B)>b$.

d) Dos dois itens anteriores, seguem-se que $\limsup _{r \rightarrow 0} f(\mathbb{B}(x, r)) \leq a$ e $\liminf _{r \rightarrow 0} f(\mathbb{B}(x, r)) \geq b$ se, e somente se, $(V) \lim \sup _{S \rightarrow x} f(S) \leq a$ e $(V) \liminf _{S \rightarrow x} f(S) \geq b$, respectivamente.

e) Se $r_{n} \downarrow 0$, então diam $\mathbb{B}\left(x, r_{n}\right) \downarrow 0$ para todo $n \in \mathbb{N}$. Reciprocamente, se $\left(S_{n}=\mathbb{B}\left(x, \rho_{n}\right)\right)_{n \in \mathbb{N}}$ é tal que diam $S_{n} \downarrow 0$, para cada $n \in \mathbb{N}$ podemos escolher, por argumento análogo ao feito acima, $0<r_{n} \leq \operatorname{diam} S_{n}$ tal que $S_{n}=\mathbb{B}\left(x, r_{n}\right)$ e, portanto, $r_{n} \downarrow 0$. Em vista da caracterização feita no item $a$ ), conclui-se que o item $d$ ) vale com as desigualdades trocadas.

O que vai acima permite concluir que, para todo $x \in X$,

$$
\limsup _{r \rightarrow 0} f(\mathbb{B}(x, r))=(V) \limsup _{S \rightarrow x} f(S) \quad \text { e } \quad \liminf _{r \rightarrow 0} f(\mathbb{B}(x, r))=(V) \liminf _{S \rightarrow x} f(S) .
$$

Teorema 3.26. Sobre o espaço métrico $(X, d)$, suponhamos uma das seguintes condições:

i) ou para $\mu$-q.t. $x \in X$ e todo $r>0, \mu(\mathbb{B}(x, r))>0$, além de

$$
\limsup _{r \rightarrow 0^{+}} \frac{\mu(\mathbb{B}(x, 5 r))}{\mu(\mathbb{B}(x, r))}<\infty
$$

ii) ou $X$ é união de uma família enumerável $\mathcal{K}$ tal que $d$ é direcionalmente limitada para cada membro de $\mathcal{K}$ e $X$ é separável.

$S e \nu \in \mathcal{M} e$

$$
W:=\left\{x \mid \lim _{r \rightarrow 0^{+}} \frac{\nu(\mathbb{B}(x, r))}{\mu(\mathbb{B}(x, r))}=\infty\right\}, \quad P:=\{x \mid \mu(\mathbb{B}(x, r))=0 \text {, para algum } r>0\} .
$$

Então $W \in \mathscr{B}_{X}, P$ é aberto em $X$ e $\nu_{\mu}=\nu\left\llcorner(W \cup P)^{c}\right.$.

Demonstração. Ver o Teorema 2.9.15 em [Fed96], juntamente com a Observação 3.25. 


\subsection{Fórmula da área em espaços métricos}

Nesta seção, combinaremos fatos sobre a teoria de diferenciação de medidas e outros resultados sobre medidas para apresentar, no Teorema 3.29, a fórmula da área para aplicações contínuas entre espaços métricos, sob suposições de regularidade mínima, feita em [Mag11] por Valentino Magnani. Esta é uma consequência de uma noção abstrata de Jacobiano introduzida na Definição 3.28, considerada como uma relação entre duas medidas. Em particular, não será exigida qualquer noção de diferenciabilidade.

Na exposição a seguir, para simplificar e deixar as ideias mais claras, consideraremos as funções e medidas definidas em $X$, ao contrário de [Mag11], onde as mesmas são definidas num subconjunto $E \subset X$ fechado fixo.

Notação. Consideraremos $(X, d, \mu)$ e $(Y, \rho, \nu)$ dois espaços métricos, onde $X$ é completo e separável, $\mu \in \mathcal{M}$ (i.e. é uma medida Borel-regular sobre $X$ finita nas partes limitadas de $X$ ), $\nu$ é uma medida de Borel sobre $Y, f: X \rightarrow Y$ contínua e $V$ uma $\mu$-relação de Vitali em $X$, como na Definição 2.29.

Para todo $S \subset X$, definimos a função $\zeta$ como $\zeta(S):=\nu(f(S))$. Denotaremos por $f^{*} \nu$ a medida que resulta da construção de Carathéodory, Teorema 1.29 , com o método $\zeta$ na família $\mathcal{F}=\mathscr{B}_{X}$. Pela Obervação 1.30, temos que $f^{*} \nu$ é uma medida Borel-regular em $X$.

Definição 3.27 (Medida Pull-Back). Sejam $(X, d)$ um espaço métrico completo e separável e $f: X \rightarrow Y$ contínua. Chamaremos $f^{*} \nu$ como a medida pull-back de $\nu$ por $f$.

Lembrando a Definição 1.34, definimos a função de multiplicidade de $f: X \rightarrow Y$ em relação a $A \subset X$ por, para todo $y \in Y, N(f, A, y):=N\left(\left.f\right|_{A}, y\right)=\#\left(A \cap f^{-1}\{y\}\right)$. Com a Definição 2.31 sobre limites ao longo de uma cobertura, introduziremos abaixo uma noção de jacobiano de $f$, através do qual será possível provar a fórmula da área no Teorema 3.29.

Definição 3.28 (Jacobiana Métrica). Sejam $f: X \rightarrow Y$ contínua e $x \in X$. A Jacobiana Métrica de $f$ em $x$ é definida por:

$$
J f(x):=(V) \limsup _{S \rightarrow x} \frac{f^{*} \nu(S)}{\mu(S)} .
$$

Teorema 3.29 (Formula da Área). Seja $f: X \rightarrow Y$ contínua. Suponha que a medida pull-back $f^{*} \nu$ seja finita nos conjuntos limitados de $X$ e que $f^{*} \nu \ll \mu$. Então Jf é $\mu$-mensurável, finita $\mu$-q.s. e, para todo conjunto boreliano $A \subset X$, temos

$$
\int_{A} J f(x) d \mu(x)=\int_{Y} N(f, A, y) d \nu(y) .
$$

Demonstração. Note que $f^{*} \nu \in \mathcal{M}$, pois é Borel-regular (em vista da construção de Carathéodory) e, pela hipótese, $f^{*} \nu$ é finita sobre conjuntos limitados de $X$. Do Teorema 3.10 segue que qualquer conjunto $\mu$-mensurável $A \subset X$ (o que vale, em particular, se $A$ boreliano) também é $f^{*} \nu$-mensurável e

$$
f^{*} \nu(A)=\int_{A} \mathrm{D}\left(f^{*} \nu, \mu, V, x\right) d \mu(x)
$$

onde $\mathrm{D}\left(f^{*} \nu, \mu, V, x\right)$ é a derivada de $f^{*} \nu$ em relação a $\mu$, definida $\mu$-q.s. em $X$ por

$$
\mathrm{D}\left(f^{*} \nu, \mu, V, x\right)=(V) \lim _{S \rightarrow x} \frac{f^{*} \nu(S)}{\mu(S)}=J f(x),
$$

sendo que a última igualdade vale $\mu$-q.s. em $X$ pela Definição 3.28 da métrica Jacobiana. Em particular, segue do Lema 3.9 e do Teorema 3.8 que $J f$ é $\mu$-mensurável e finita $\mu$-q.s. 
Por outro lado, para qualquer boreliano $A \subset X$, segue do Teorema 1.35 (o qual pode ser aplicado, tendo em vista que, para todo $A \in \mathscr{B}_{X}, f(A)$ é $\nu$-mensurável pela Proposição 1.33 e pelo fato de que $X$ é espaço métrico completo separável, $Y$ é Hausdorff e $f$ é contínua) que $N\left(\left.f\right|_{A}, \cdot\right)$ é $\nu$-mensurável e

$$
f^{*} \nu(A)=\int_{Y} N\left(\left.f\right|_{A}, y\right) d \nu(y)
$$

donde a tese.

Observação 3.30. Uma outra versão da fórmula da área também é provada em [Mag11], a qual se baseia numa noção modificada de jacobiano métrico. 


\section{Referências Bibliográficas}

[Bes45] Abram S. Besicovitch. A general form of the covering principle and relative differentiation of additive functions. Em Mathematical Proceedings of the Cambridge Philosophical Society, volume 41, páginas 103-110. Cambridge University Press, 1945. xi, 22

[Bes46] Abram S. Besicovitch. A general form of the covering principle and relative differentiation of additive functions. II. Em Mathematical Proceedings of the Cambridge Philosophical Society, volume 42, páginas 1-10. Cambridge University Press, 1946. xi, 22

[BI01] Dmitri Burago; Yuri Burago e Sergei Ivanov. A Course in Metric Geometry, volume 33. American Mathematical Soc., 2001. 1

[BL16] Blanche Buet e Gian Paolo Leonardi. Recovering measures from approximate values on balls. Annales Academiae Scientiarum Fennicae Mathematica, 41:947-972, 2016. 17, 22

[dC15] Manfredo P. do Carmo. Geometria Riemanniana. IMPA, quinta edição, 2015. 17

[Dom14] Xavier Tolsa Domènech. Teoría geométrica de la medida e integrales singulares. Gaceta de la Real Sociedad Matematica Española, 17(2):361-382, 2014. xi

[EG91] Lawrence C. Evans e Ronald F. Gariepy. Measure Theory and Fine Properties of Functions. Studies in Advanced Mathematics. Taylor \& Francis, 1991. xi, 1, 2, 13, 22

[Fed96] Herbert Federer. Geometric Measure Theory. Classics in mathematics. Springer, 1996. xi, $1,2,3,4,6,7,8,9,11,15,17,23,26,43,44$

[Fol99] Gerald B Folland. Real analysis: modern techniques and their applications. John Wiley \& Sons, Inc., New York, 1999. Second edition. 1, 6

[Hei01] Juha Heinonen. Lectures on analysis on Metric Spaces. Universitext. Springer, 2001. 22

[Ito18] Tsubasa Itoh. The Besicovitch covering theorem for parabolic balls in Euclidean space. Hiroshima Math. J., 48(3):279-289, 2018. 17, 23

[Lim70] Elon Lages Lima. Elementos de Topologia Geral. Textos universitários. SBM, 1970. 1

[Mag11] Valentino Magnani. An area formula in Metric Spaces. Colloquium Mathematicum, 124(2):275-283, 2011. xii, 45, 46

[Mat95] Pertti Mattila. Geometry of sets and measures in Euclidean spaces, volume 44 of Cambridge Studies in Advanced Mathematics. Cambridge University Press, Cambridge, 1995. Fractals and rectifiability. 1, 4, 22

[Rig18] Severine Rigot. Differentiation of measures in Metric Spaces. arXiv preprint arXiv:1802.02069, 2018. xi, 23

[Sil11] João Vitor da Silva. Teoria Geométrica da Medida e Aplicações. Dissertação de Mestrado, Universidade Federal do Ceará, 2011. xi 
[Sim83] Leon Simon. Lectures on geometric measure theory, volume 3 of Proceedings of the Centre for Mathematical Analysis. Australian National University, Centre for Mathematical Analysis, Canberra, 1983. 13

[Sim14] Leon Simon. Introduction to geometric measure theory, volume 91. Lectures on geometric measure theory (Canberra, 1983), revised edition, 2014. 25

[Tal13] Mohammad Talebi. Covering Theorems, 2013. URL:http://aurora.asc.tuwien.ac.at/ funkana/downloads_general/sem_talebi.pdf. 22

[Ter19] Gláucio Terra. Introduction to Geometric Measure Theory - Lecture Notes Version: 1.6, 2019. URL:https://www.ime.usp.br/ glaucio/mat6704/textos/GMTLecureNotes.pdf. 4, $5,6,34$

[Tor19] Tatiana Toro. Geometric measure theory. Notices of the American Mathematical Society, 66(4), 2019. xi 